University of Louisville

ThinkIR: The University of Louisville's Institutional Repository

Electronic Theses and Dissertations

$11-2011$

\title{
Quantification of vascular perfusion in the spinal cord after injury.
}

Ruthie S. Fligor

University of Louisville

Follow this and additional works at: https://ir.library.louisville.edu/etd

\section{Recommended Citation}

Fligor, Ruthie S., "Quantification of vascular perfusion in the spinal cord after injury." (2011). Electronic Theses and Dissertations. Paper 443.

https://doi.org/10.18297/etd/443

This Master's Thesis is brought to you for free and open access by ThinkIR: The University of Louisville's Institutional Repository. It has been accepted for inclusion in Electronic Theses and Dissertations by an authorized administrator of ThinkIR: The University of Louisville's Institutional Repository. This title appears here courtesy of the author, who has retained all other copyrights. For more information, please contact thinkir@louisville.edu. 


\title{
QUANTIFICATION OF VASCULAR PERFUSION IN THE SPINAL CORD AFTER INJURY
}

\author{
By \\ Ruthie S. Fligor \\ B.S., University of Louisville, 2011
}

\author{
A Thesis \\ Submitted to the Faculty of the \\ Speed School of Engineering of the University of Louisville \\ As Partial Fulfillment of the Requisites \\ For the Professional Degree of
}

\section{MASTER OF ENGINEERING}

Department of Bioengineering

University of Louisville

Louisville, Kentucky

NOVEMBER, 2011 
SIGNATURE PAGE

QUANTIFICATION OF VASCULAR PERFUSION IN THE SPINAL CORD AFTER INJURY

BY

Ruthie S. Fligor

University of Louisville

A Thesis Approved on

(DATE)

By the following Thesis Committee

Andre M. Gobin, PhD, Thesis Director

David Magnuson, $\mathrm{PhD}$ - Thesis Advisor

Richard L. Benton, $\mathrm{PhD}$ - Thesis Committee 
Palaniappan Sethu, PhD - Thesis Committee

Stuart J. Williams, PhD - Thesis Committee 


\title{
ABSTRACT \\ QUANTIFICATION OF VASCULAR PERFUSION IN THE SPINAL CORD AFTER INJURY
}

\author{
Ruthie S. Fligor \\ November, 2011
}

Traumatic injury destroys blood vessels at the injury epicenter and is followed by local angiogenesis and regional inflammation. Healing from injury depends on vascular health because blood supply is directly responsible for the health and function of surrounding tissue. This work establishes a new method for qualitatively and quantitatively measuring the blood supply of spinal cord (SC) tissue. Systemically injecting fluorescent microspheres (FMs) and cryostat sectioning SC tissue reveals a novel and potentially powerful way of assessing blood supply. This method is easily incorporated with existing tissue processing protocols because it does not require chemical digestion of the tissue region of interest. FM blood supply measurements show that after mild contusion injury, the epicenter has less blood flow while the blood flow several millimeters rostral and caudal to the epicenter is elevated compared to uninjured controls. The time course for vascular repair after spinal cord injury (SCI) has been widely studied and this pilot experiment was carried out seven days post-injury, at which point angiogenesis has reached its zenith and vascular pruning is minimal. A custom MATLAB program is used to automatically analyze FM distribution. 


\section{TABLE OF CONTENTS}

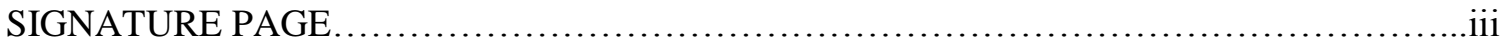

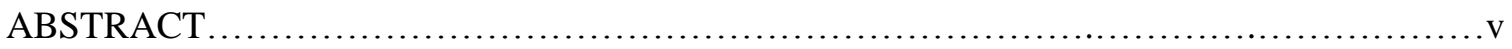

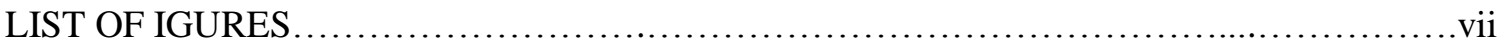

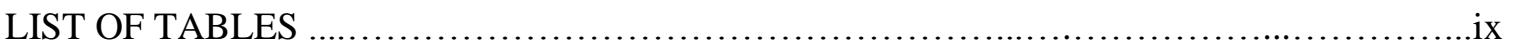

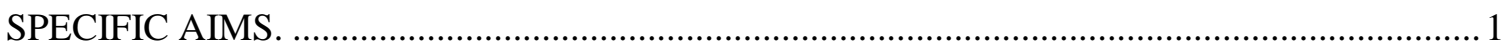

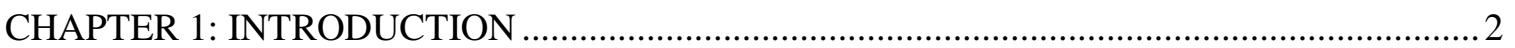

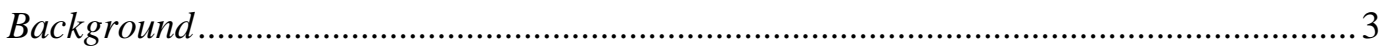

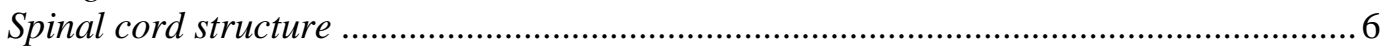

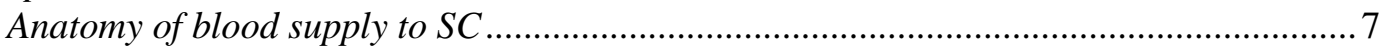

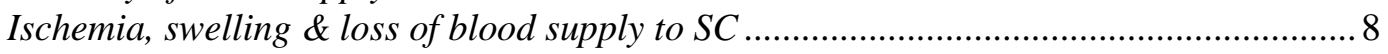

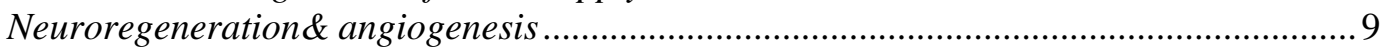

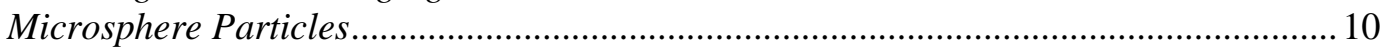

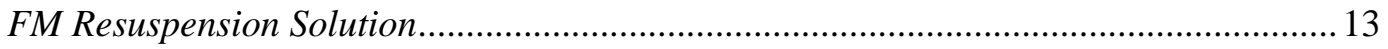

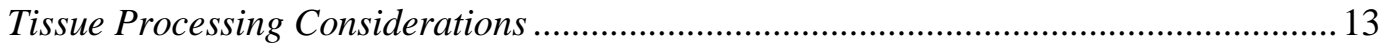

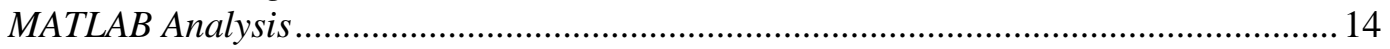

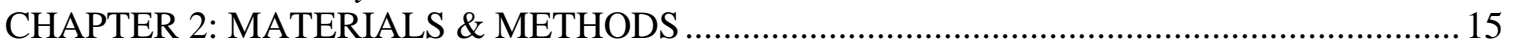

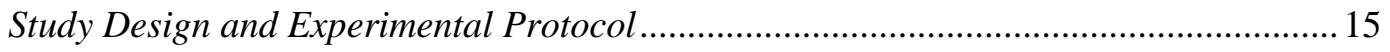

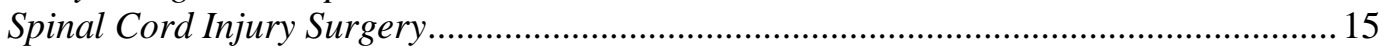

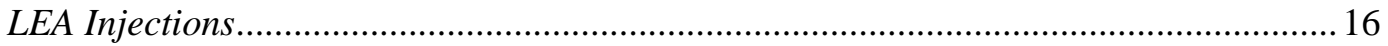

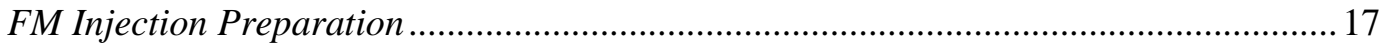

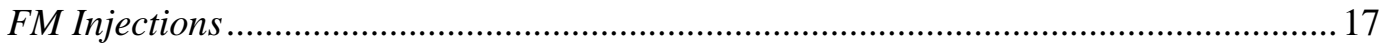

Tissue Processing \& Histological Analysis ................................................................ 19

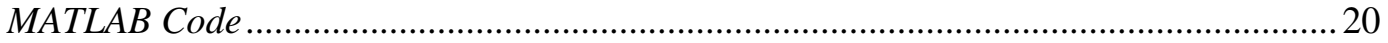

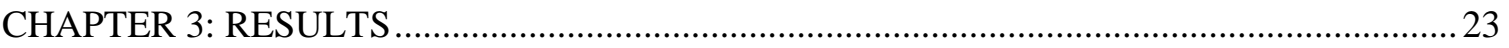

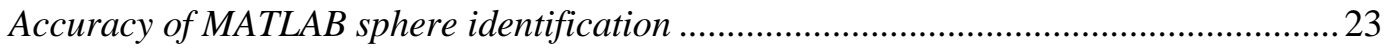

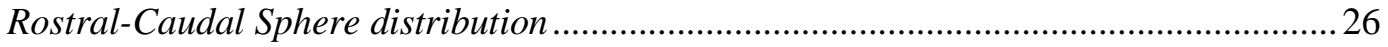

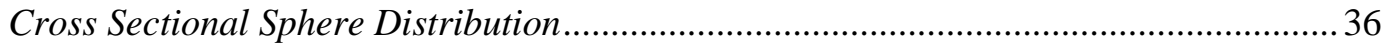

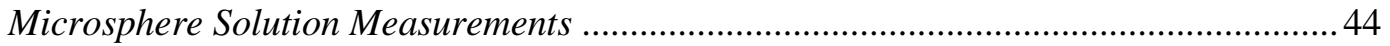

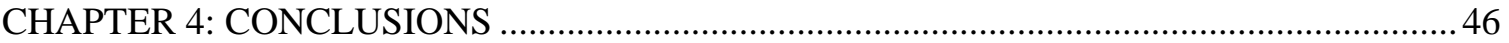

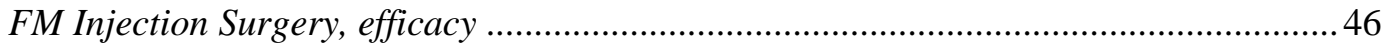

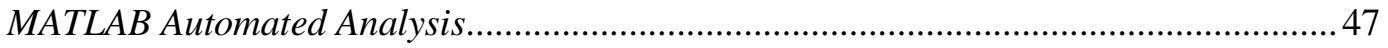

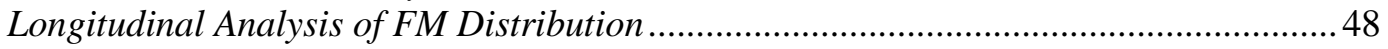

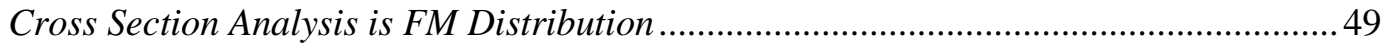

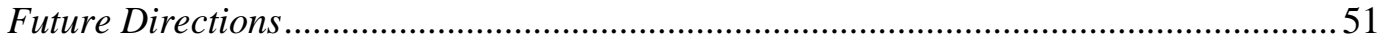

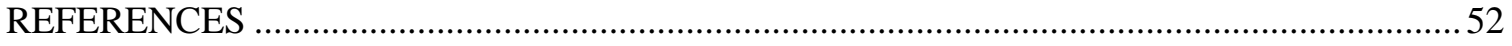

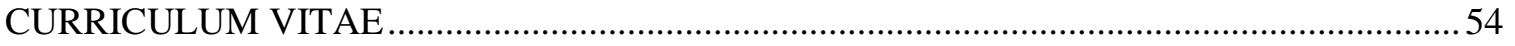

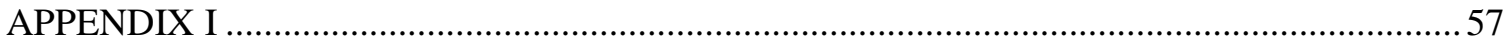

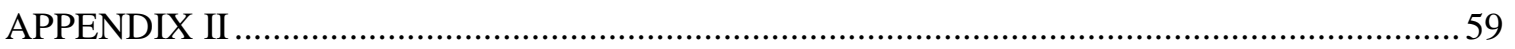

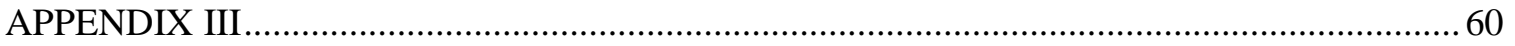

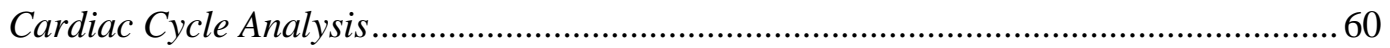

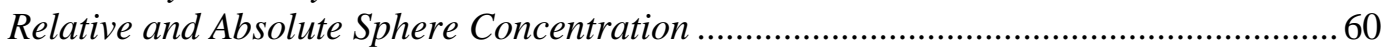

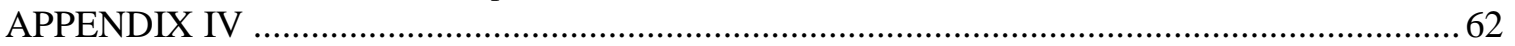




\section{LIST OF FIGURES}

FIGURE

Page

Figure 1: Fluorescent microspheres embolized in cardiomyocyte tissue; fluorescence is excited with laser light but images were published as grayscale. Two sequentially injected colors are used to generate a vector, indicating the direction in which that blood vessel's flow is directed (Cicutti and Rakusan 1994).

Figure 2: Photograph of Lumber level 2 spinal cord, depicting white and grey matter

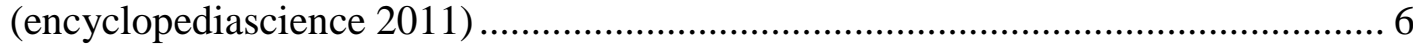

Figure 3: (A) Spinal cord blood supply and venous drainage; (B) Saggital cross section of blood supply illustrating the small vessels branching from a circumferential artery and penetrating inward, or a larger trunk forging inward from the anterior spinal artery and branching out. (2004) ...

Figure 4: (A) Percent of new axons "Following Blood Vessels" (FBV) at various days after SCI; (B) An example of axon sprouts growing along the dorsal vein, 118 days after injury (Dray, Rougon et al. 2009).

Figure 5: Excitation and emission spectra for all Spherotech fluorescent particles; UV particles (which appear as blue) are highlighted with blue and purple particles (which fluoresce red) are highlighted with red. ....................................................... 11

Figure 6: Polystyrene structure.............................................................................. 12

Figure 7 Illustration of initiator and styrene groups for particles less than $3 \mu \mathrm{m}$ in

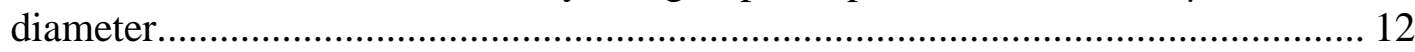

Figure 8 Initiator and styrene groups for particles greater than $3 \mu \mathrm{m}$ in diameter.......... 12 Figure 9: For some images, the lab members counted more FMs than MATLAB. Neither of the two thresholds tested is more accurate in counting FMs in these images.

Figure 10: For some images, the "true" count is very close to the measured counts. For a few images, there is a large difference................................................................ 25

Figure 11: Statistical analysis showing that the difference between MATLAB counting and human counting (DIFF) is not significant and was estimated from images with no apparent inherent bias. 12 
Figure 19: Longitudinal analysis of injured SC, Animal 3, each consecutive 2 points averaged; two types of lines added with MATLAB for comparison ....................... 30

Figure 20: Longitudinal analysis of uninjured SC Animal 4, each 2 consecutive images averaged.

Figure 21: Longitudinal analysis of uninjured SC, each 2 consecutive points averaged 31 Figure 22: Longitudinal analysis of uninjured SC, Animal 6, each 2 consecutive points averaged

Figure 23: Animal 1, averaged by 5's; this is likely over-simplifying the data.............. 32

Figure 24: Animal 1, averaged by 2's .............................................................. 32

Figure 25: Animal 2, averaged by 10's; direct fit line applied, rather than a polynomial 33

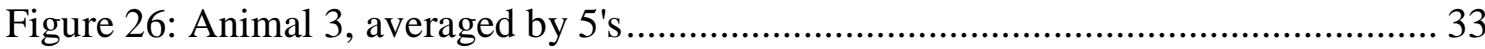

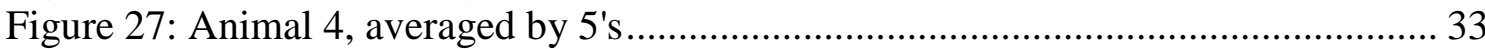

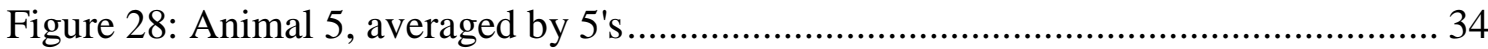

Figure 29: Animal 6, averaged by 5's ................................................................ 34

Figure 30: Injured animal data (blue) compared to uninjured trend (red). Y-axis reflects average FM count; $x$-axis corresponds to distance in $4 \mathrm{~mm}$ increments ................ 36

Figure 32: The same 10 images are analyzed using different colormap bin sizes. The information being presented is exactly the same, but it appears different and different assumptions might be made based on how the colormap appears. As the bins get smaller, it is increasingly likely that only one or two FMs will be detected per bin, so the distribution (blood supply) seems less intense.

Figure 33 (next 3 pages): Animal 4 (left side) is an uninjured control and Animal 2( right side) is an injured animal. This figure displays level-matched color plots of corresponding tissue from each animal. Animal 4 was imaged as 2 of the 5 sets, while Animal 2 was imaged in its entirety; therefore the image numbers printed above each colormap correspond but do not match precisely. While there are 2.5 times more images for Animal 2, this does not explain the increased number of FMs detected because the colors on the plot are scale automatically. Apparent blood flow patterns are not affected by the different numbers of images analyzed. ......... 37

Figure 34: Colorplot of Animal 2. FM count is labeled on the z-axis. The $\mathrm{x}$ - and y-axis correlate only to image orientation which is shown here with the anterior edge near and the posterior side in the background

Figure 35: Colorplot of Animal 4. Axis are identical to Figure 35; the anterior direction is oriented in the foreground and slightly to the right.

Figure 36: Uninjured spinal cord, before being cut into 4 even blocks. The third block is the tissue analyzed in this study. It contains the lower thoracic and upper lumbar segments. T9 is approximately at the center of the red block.

Figure 37: Colormap illustration of trends in FM distribution across injury epicenter .. 43

Figure 38: Particle size distribution for "1.8 micrometer diameter" particles ................ 45

Figure 39: Zeta potential measurements for 1.8 micrometer particles ........................ 45

Figure 40: Vasculature versus Blood Flow - (A) structure, adapted from Anesthesia UK

(B) blood flow as measured in this study (C) blood flow re-colored with vascular pattern overlay, and approximate region supplied with blood by the posterior arteries (D) and anterior artery (E)....

Figure 41 Surface area, absolute count, and particle volume formulas provided by Spherotech 


\section{LIST OF TABLES}

Table 1: Tests done prior to shipping FM solutions. ................................................. 11

Table 2: MATLAB features which are conveniently adjusted by the user..................... 22

Table 3: Comparison of FM distribution in injured and uninjured animals, by region .... 35

Table 4: Precise tissue designations defining the each region for color plot analysis; image file numbers are recorded on top, and below that is the calculated distance spanned by each segment in microns and italicized .......................................................... 42

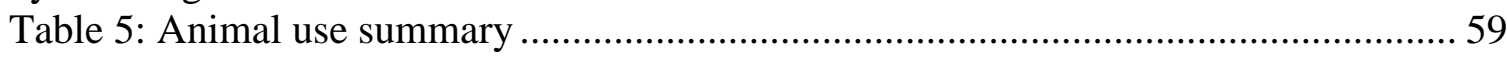




\section{SPECIFIC AIMS}

The goal of this work is to develop a novel system to quantitatively analyze the angiogenesis that occurs in and around the epicenter of a spinal cord injury. This system will incorporate advanced microscopy and imaging techniques.

1) Develop Surgery: Perform and document a repeatable surgical protocol for administration of fluorescent microspheres (FMs) to adult rats in a terminal surgical model, showing that FMs lodge in SC tissue frequently enough that reliable quantification can be done.

2) Show cell-perfusion of vasculature: Use FM probes to identify regional patterns of cell perfused vasculature in injured and uninjured spinal cord tissue.

3) Automate analysis: Develop custom MATLAB programs to analyze distribution patterns of fluorescent particles both (A) longitudinally (rostral to caudal) and (B) regionally within sagittal sections of spinal tissue. 


\section{CHAPTER 1: INTRODUCTION}

After physical damage, macrovessels and microvessels undergo changes in response to cytokine signals from damaged cells. As the tissue recovers and repairs from an injury, local vasculature undergoes a period of angiogenesis followed by pruning. Once it is fully stabilized the new vascular network's pattern will correlate with areas of healthy tissue. The collagen fibers which compose scar tissue receive less blood flow than tissue made of regenerated or repaired cells (Thomas K 1988; Fawcett and Asher 1999; Tonnesen, Feng et al. 2000). In this work, "blood supply" is considered the physical structure of the vasculature and also, as a separate concept, the directed flow of plasma and blood cells through vascular structures. This distinction is important because there should be no assumption that vasculature which is physically apparent using histological staining is necessarily functional and perfused with cells.

Acute injury to the central nervous system destroys neuronal tissue as well as regional blood supply. Vascular damage is associated with numerous issues related to secondary injury and neuronal cell death (Awad, Ankeny et al. 2010). Angiogenesis near the injury epicenter is closely correlated with repair and regrowth of neuronal tissue (Dray, Rougon et al. 2009). In turn, population of the injury site with healthy cells is correlated with functional recovery (Urdzíková, Jendelová et al. 2006). However, a directly causal effect relating increased angiogenesis to better functional outcome after central nervous system (CNS) damage has not been conclusively shown.

This work seeks to establish microsphere embolization as useful for measuring relative blood supply in the spinal cord in our rat model of spinal cord injury. Microsphere particle injection is surgically paired with fluorescein isothiocyanate (FITC)-conjugated Lycopersicon esculentum agglutinin (tomato) lectin (LEA) perfusion. The lectin technique is already used in 
this center (Benton, Maddie et al. 2008) and is established as a reliable technique for systemic blood vessel identification.

Lectin staining and fluorescent microsphere perfusion are administered in the same animals to compare apparent vascular patterns reflected by the two markers. Imaging programs have already been developed and characterized for analysis of lectin stained vasculature. Here, a custom MATLAB program is developed and used to measure fluorescent microsphere distribution in spinal tissue. The program enables precise analysis of embolization patterns, which are reflective of relative blood flow.

Quantifying cell perfused vasculature with fluorescent microspheres is a relatively inexpensive outcome measure, does not call for expensive equipment, and does not require chemical digestion of the tissue, so other important histological and immunological tests may be done on the same sample.

\section{Background}

Beginning in the late 1960 's, injections of radiolabeled microspheres were compared with simultaneous blood draws and with radioactivity of tissue biopsies to measure blood flow in a region or tissue (Domenech, Hoffman et al. 1969). The premise behind this method lies in the fact that any vasculature smaller than a microsphere will be embolized by that particle, and the particle will be arrested prior to moving into the venous portion of the vascular network. Several groups have demonstrated that the number of spheres lodged in tissue is directly proportional to the blood flow received by that tissue (Alm and Bill 1973). Decades later, fluorescent microparticles (FMs) were established as a safer, cheaper, and more stable alternative to radiolabeled spheres (Morita, Payne et al. 1990). However, both relative and absolute blood flows were still calculated by digesting the tissue of interest and examining the number of remaining fluorescent particles using filtration or spectrophotometry. Analysis of absolute blood 
flow furthermore requires a controlled blood draw in addition to tissue analysis (De Visscher, Haseldonckx et al. 2006).

The technique developed in the present work is more useful in the field of spinal cord injury because it allows histological and immunohistochemical analysis to be done on tissue samples. It uniquely incorporates fluorescent particle considerations with standard tissue stains by analyzing FMs after cryostat sectioning. The tissue itself is not altered (digested) as part of the counting process. Precise histological analysis of cross-sectioned injury epicenters requires tissue staining and is required to measure the severity of the injury in animal models (Scheff, Rabchevsky et al. 2003; Magnuson, Lovett et al. 2005). Correlation of functional outcomes would not be useful without measuring injury severity, so eliminating the need for tissue digestion (the most common means of measuring fluorescent particles) makes this an attractive blood supply measurement tool.

Buckberg et al. describe the minimum sample size of microparticles in a specific region of interest (ROI) required for statistical validation that the sphere measurement reflects actual blood flow rate (Buckberg, Luck et al. 1971). They establish that at least 400 spheres should be found in an ROI; this has since become a widely-cited standard. These authors state that spheres approaching $8 \mu \mathrm{m}$ in diameter have a favorable tissue distribution compared to spheres with diameters in the $14-50 \mu \mathrm{m}$ ranges. They specifically note that "they are distributed more like red blood cells, occlude less of the vascular bed ... allowing more reliable measurements of flow to smaller regions."

Other protocol considerations were drawn from Development of a novel fluorescent microsphere technique to combine serial cerebral blood flow measurements with histology in the rat (De Visscher, Haseldonckx et al. 2006) as well as procedures published by Rakusan et al. (Cicutti and Rakusan 1992; Cerutti, Gustin et al. 2001). Rakusan et al. used $10 \mu \mathrm{m}$ diameter spheres to achieve embolization of microvessels in cardiac muscle. One technique they developed was to inject a second solution of spheres which were conjugated with a different 
fluorophore, then use co-occurrence of the two types to identify which end of the embolized vessel was receiving arterial blood and which end was directed towards the capillary bed and venous system (see Figure 1). When infusing the whole body with FMs, this group and others document using approximately $6 \times 10^{6}$ total sphere particles. This is far more than the $1 \times 10^{6}$ particles per infusion suggested by de Visscher, but the high number of particles was documented to not have a measurable change in the animal's vital signs (heart rate and breathing rate) and was required for statistical accuracy of the results.

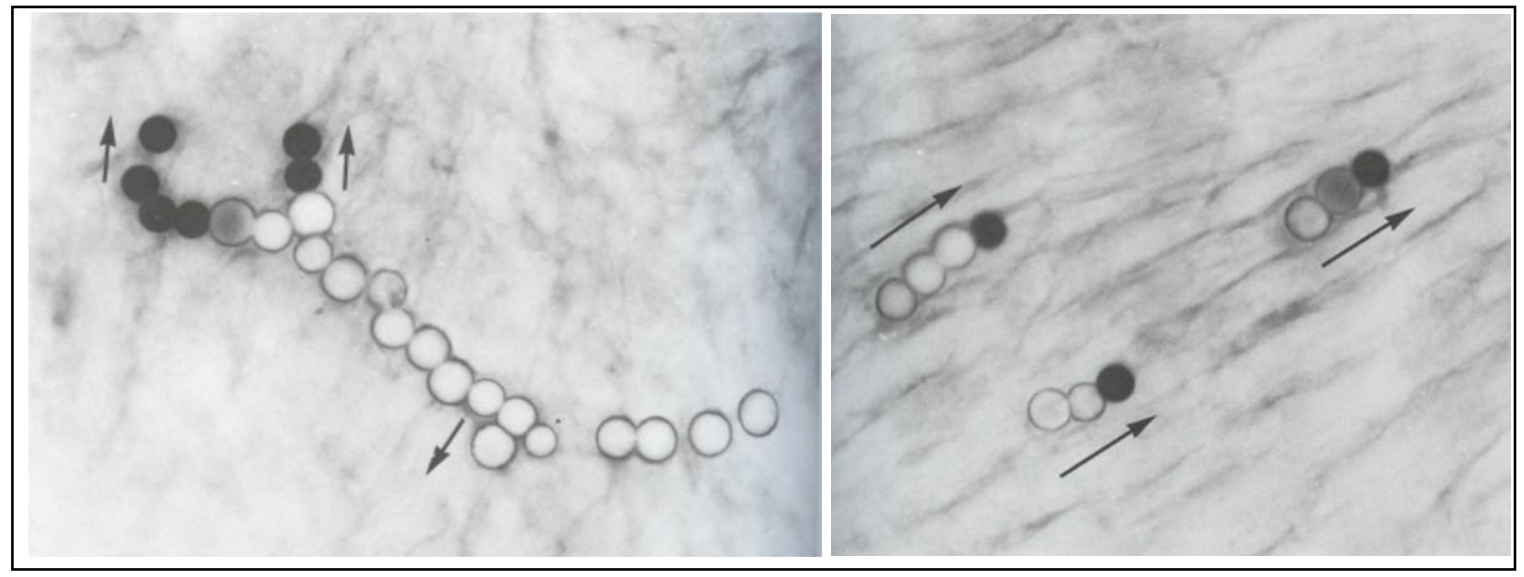

Figure 1: Fluorescent microspheres embolized in cardiomyocyte tissue; fluorescence is excited with laser light but images were published as grayscale. Two sequentially injected colors are used to generate a vector, indicating the direction in which that blood vessel's flow is directed (Cicutti and Rakusan 1994).

Pioneers of this technique have noted that if the particles do not mix thoroughly with the flowing blood then the sphere-labeling may have some bias and will not be indicative of true blood supply. This is typically overcome by accessing the artery with the least laminar flow: the ascending aorta. Past work has shown that either a bolus injection or a slow pump-infusion of FMs in the left ventricle or just past the aortic valve is effective in delivering particles to biopsied tissues in proportion to the amount of blood flow that the tissue was receiving. FMs have been used to study relative blood flow in ischemic brain tissue (Yamakami and McIntosh 1989), and radioactive microparticles were used for blood flow measurements in the spinal cord (Marcus, 
Heistad et al. 1977). Use of FMs to study relative cell perfusion in a murine model of spinal cord injury is an extension of these works.

\section{Spinal cord structure}

The central nervous system (CNS) is composed of the brain and the spinal cord. The CNS is anatomically distinct from the peripheral nervous system. The spinal cord lies inside the vertebral column where, in the rat, the individual vertebrae are classified as 7 cervical, 13 thoracic, 6 lumbar, 4 sacral, and 27-31 coccygeal bones. (Human spinal columns, for comparison, consist of 7 cervical, 12 thoracic, 5 lumbar, 5 fused sacra, and 4 fused coccygeal vertebrae.) Thoracic vertebrae increase in length from T1 to T13 and range from $2 \mathrm{~mm}$ to $7 \mathrm{~mm}$ long. An intact spinal column is stabilized by ligaments connecting the vertebral bodies and intervertebral disks. Within the vertebral column, the spinal cord itself is considerably shorter than the bony structure so the L1 spinal cord level is located at the T10 level vertebra (Hebel and Stromberg 1986). Levels are similarly mismatched in the lower spinal regions in humans (Somers 2001).

The spinal cord has morphologically distinct regions of grey matter and white matter. White matter is composed mostly of myelinated axons; it appears white because myelin is 70$85 \%$ lipid (dry mass). Grey matter is a combination of neuronal cell bodies, glia, and capillaries.

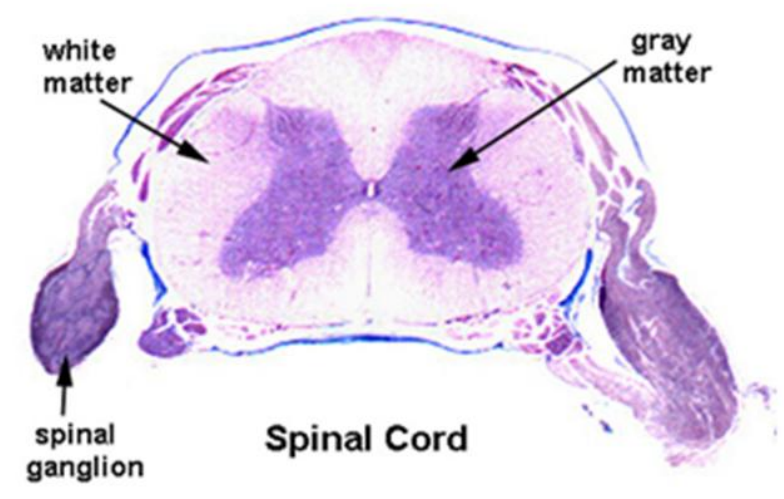

Figure 2: Photograph of Lumber level 2 spinal cord, depicting white and grey matter (encyclopediascience 2011) 
Its visually distinct color is partially attributed to the presence of blood and blood capillaries. In cerebral and cerebellar cortices the grey matter is on the outside and white matter is on the inside. This pattern is reversed within the low brainstem region. White matter shifts to the perimeter of the spinal cord while grey matter forms a distinctive " $\mathrm{H}$ " or "butterfly" shape and can be seen clearly in spinal cord cross sections (Figure 2). The exact shape of the grey matter within the cord is so predictable that grey matter shape alone is enough to identify the spinal level to which a cross section sample belongs. A cerebrospinal fluid-fill lumen called the central canal runs longitudinally down the entire spinal cord (Purves, Augustine et al. 2001; Somers 2001). The central canal is frequently used as a landmark for aligning tissue segments during imaging.

\section{Anatomy of blood supply to SC}

The blood supply of the spinal cord, illustrated in Figure 3, has developed as two simultaneous approaches to tissue perfusion: it circumvents the cord then branches inward, or it forges directly in and then branches outward. The primary supply consists of two posterior spinal arteries (arteria spinalis posterior) and one anterior spinal artery (arteria spinalis anterior). The two posterior vessels are located several degrees lateral of midline and, together, supply roughly one third of the posterior portion of the cord with blood. The anterior spinal artery is on the midline of the anterior cord surface. Its branches bring blood to both the central cord (from the inside) and to the more ventral white matter (from the outside) and it supplies roughly twice as much tissue by volume as the two posterior arteries combined (Dommisse 1974).

These main arteries receive blood from the subclavian artery (arteria subclavia) which is rooted near the aortic arch. There is some conflict regarding exactly how much of the blood to the spinal cord is provided by these large arteries. Some work suggests that they are the main blood supply of the spinal cord from the cervical level all the way to the lumbar region (Awad, Ankeny et al. 2010). Other literature suggests that considerable blood is provided by small 
radicular arteries which stem from segmental arteries, otherwise known as intercostal and lumbar arteries (Biglioli, Roberto et al. 2004).

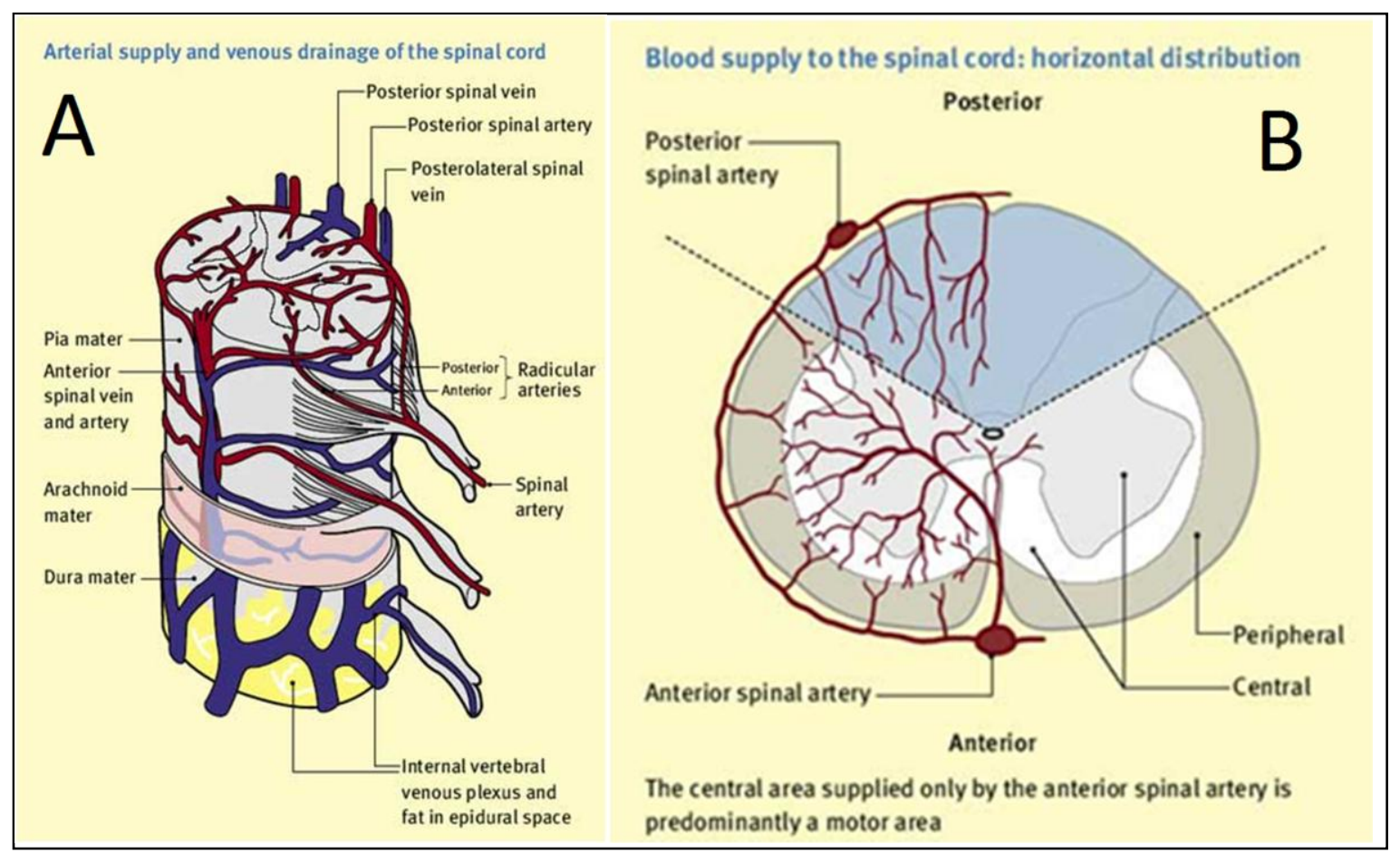

Figure 3: (A) Spinal cord blood supply and venous drainage; (B) Saggital cross section of blood supply illustrating the small vessels branching from a circumferential artery and penetrating inward, or a larger trunk forging inward from the anterior spinal artery and branching out. ("Spinal Cord", 2004)

Ischemia, swelling \& loss of blood supply to SC

Most of the 10,000 annually reported SCI cases in the United States are due to traumatic injury and occur in otherwise healthy young adults. Acute trauma to the delicate spinal cord causes intense blood vessel and neural-cell damage. The hemostasis and acute inflammation responses cause the spinal cord to swell, filling its entire cavity within minutes.

Damaged vertebra at the injury site can enable swelling and inflammation to occurr locally without impedence. However, swelling also takes place well beyond the injury site. As tissue throughout the cord becomes inflammed in response to damage, the rigid vertebral canal is 
unable to expand, resulting in compression of the tissue. As compression pressure increases compared to blood pressure, less blood is able to get to the inflammed tissue, exascerbating the ischemia already underway due to direct vascular damage from the original injury (McDonald and Sadowsky 2002).

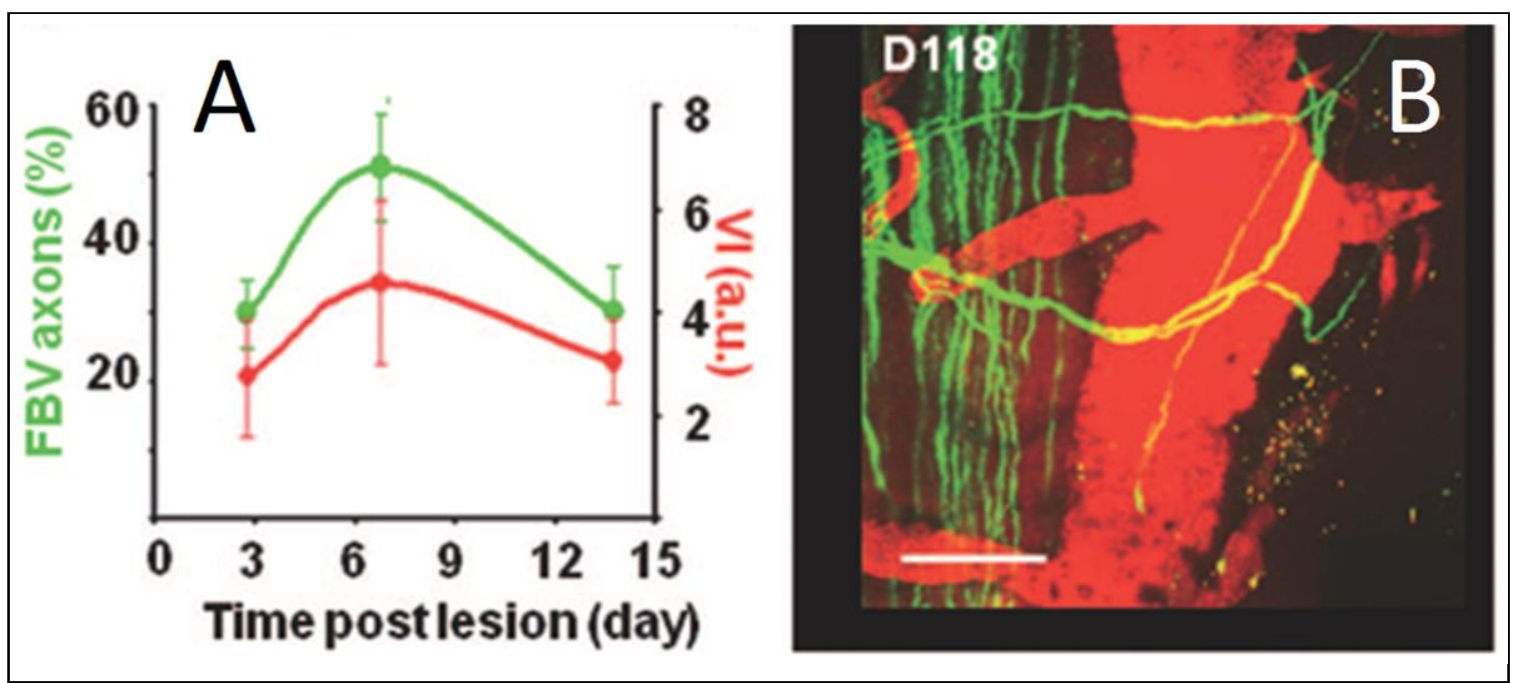

Figure 4: (A) Percent of new axons "Following Blood Vessels" (FBV) at various days after SCI; (B) An example of axon sprouts growing along the dorsal vein, 118 days after injury (Dray, Rougon et al. 2009)

\section{Neuroregeneration \& angiogenesis}

Neuroregeneration and neurodegenerative diseases have been shown to correlate with microscopic angiogenic patterns so closely that the conceptual "neurovascular unit" is an increasingly popular target for research and intervention therapies (Dray, Rougon et al. 2009). See Figure 4 for an example of work reflecting neurovascular unit changes after SCI. This work used a 2-photon microscope to follow axon sprouts and correlate angiogenic patterns with changes near SCI epicenter. The limitation here is that the imaging technique can only penetrate a few hundred microns into the SC tissue. Therefore only the white matter can be studied with this tool.

Brain and spinal cord angiogenesis are distinct from angiogenesis in vascular elsewhere because the CNS vasculature forms the blood-brain barrier (BBB). $B B B$ refers to the unique endothelial cell behavior observed in blood vessels in the brain and spinal cord; some literature 
makes a distinction between the $\mathrm{BBB}$ and the blood spinal cord barrier (BSB). The BBB/BSB is not literally a unique membrane between the CNS and blood. Rather, it is a name for the decreased permeability of endothelium in CNS vasculature compared to vascular endothelium elsewhere in the body. Permeability is reduced by the endothelial cells' formation of tight junctions and by glial cell presence surrounding CNS capillaries.

Vascular permeability and BBB cell behavior are relevant to spinal cord injury because they are largely responsible for the differences in CNS vasculature compared to peripheral tissue vessels. Angiogenic trends in the spinal cord cannot be directly predicted from angiogenesis studies on surface wound healing or even from traumatic brain injury studies.

The relationships between angiogenesis and neuronal cell health are widely studied. The temporal relationships in a rat model of SCI reflect trends in inflammation, angiogenic markers, vascular pruning, and functional recovery (Loy, Crawford et al. 2002).

\section{Microsphere Particles}

The fluorescent particles used in this study were manufactured by Spherotech, a Chicagobased microparticle manufacturer. The company specializes in microparticle development and research and offers particles in a wide range of sizes and physical properties. Fluorescent particles are stocked from 0.04 micrometers to 35 micrometers in diameter; custom sizes, including nanoparticles, are available. They also routinely supply surface-conjugated particles with functionalized groups including, but not limited to, antibodies, amino groups, carboxyl groups, and covalently coupled proteins.

The particles are described as nonporous, uniformly shaped spheres. They are inert to common lab solutions such as alcohol and DMSO but are dissolved by chemicals including DMF, acetone, acetonitrile, chloroform, methylene chloride, and xylene. Xylene, in particular, is commonly used in tissue staining protocols and should be carefully avoided. 
For the specific particles used in this work, quality control tests done by Spherotech include:

\begin{tabular}{|l|l|}
\hline \multicolumn{1}{|c|}{ Test } & \multicolumn{1}{c|}{ Results } \\
\hline Testing size using Laser Particle Sizer & Reported to client \\
\hline Testing CV\% using flow cytometer & Reported to client \\
\hline Testing \% singles by flow cytometry & Reported to client \\
\hline $\begin{array}{l}\text { Testing fluorescence using the FITC, PE, and } \\
\text { PE-Cy5 channels of a flow cytometer }\end{array}$ & $\begin{array}{l}\text { The linear channel numbers were required to } \\
\text { be within 10\% of specifications set based on } \\
\text { the initial master lot produced. }\end{array}$ \\
\hline $\begin{array}{l}\text { Determination of solid content (weight/volume } \\
\%) \text { by drying }\end{array}$ & Results reported to client \\
\hline
\end{tabular}

Table 1: Tests done prior to shipping FM solutions.

Results of company-reported testing are included in Appendix I for each type of particle used.

Information related to the exact fluorophore used in the particles is not public, but excitation and emission spectra for the beads are shown in Figure 5. Spherotech states that the fluorophore will not leach out of intact particles or react with the environment. The color designated "UV" on the chart corresponds with standard DAPI laser and filter specifications $(345 \mathrm{~nm}-455 \mathrm{~nm})$ and the color designated "Purple" corresponds with Texas Red (589nm615nm). When imaged, the UV and Purple spheres appear blue and red, respectively, and are referred to as "blue" or "red" throughout this work. See Figure 5 for spectrum illustration.

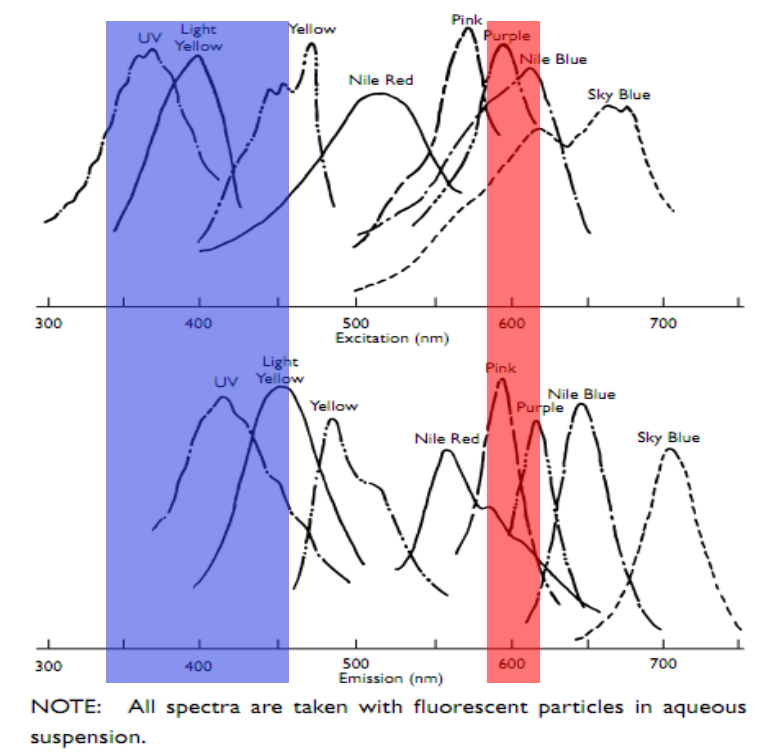

Figure 5: Excitation and emission spectra for all Spherotech fluorescent particles; UV particles (which appear as blue) are highlighted with blue and purple particles (which fluoresce red) are highlighted with red. 
The spheres are manufactured from a solid polystyrene core which is coated with a polystyrene \& fluorophore mixture. Polystyrene is an aromatic polymer of repeating styrene units; see Figure 6. Polystyrene is a solid at room temperature and has a glass transition temperature of $100^{\circ} \mathrm{C}$.<smiles>[Y]CC(CC(CC(CC(CC(CC(CC(C)c1ccccc1)c1ccccc1)c1ccccc1)c1ccccc1)c1ccccc1)c1ccccc1)c1ccccc1</smiles>

Figure 6: Polystyrene structure

Spherotech fluorescent spheres are suspended in deionized water (plus $0.02 \%$ sodium azide as a bacteriostatic). Spheres less than $3 \mu \mathrm{m}$ are manufactured using potassium persulfate as an initiator. As a result, negatively charged sulfate groups are on the surface of the spheres and the resulting particles are hydrophilic, as shown in Figure 7. Larger spheres are made with a potassium persulfate initiator, leaving uncharged benzyl rings and slightly polar hydroxyl groups on the surface of particles. This is illustrated in Figure 8 and Spherotech describes larger particles as "relatively hydrophobic".
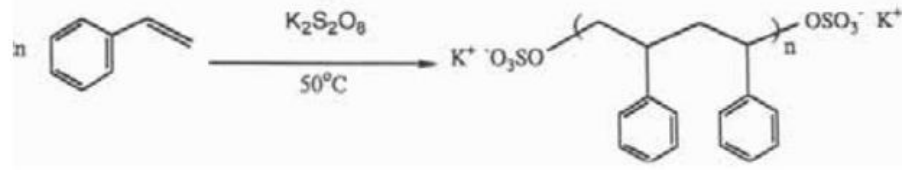

Figure 7: Illustration of initiator and styrene groups for particles less than $3 \mu \mathrm{m}$ in diameter.

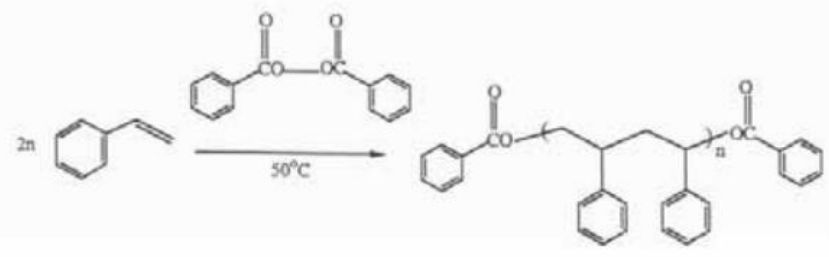

Figure 8: Initiator and styrene groups for particles greater than $3 \mu \mathrm{m}$ in diameter. 


\section{FM Resuspension Solution}

If particles have an affinity for one another, they are likely to clump together. This is undesirable in the injections described because clumping would lead to FM aggregation in blood vessels and the individual particles could not be considered to be randomly distributed. Random distribution is specifically required in this type of assay (Buckberg, Luck et al. 1971). It is assumed that particles embolize in blood vessels which have a diameter very near the diameter the particles. If the particles aggregate, they could embolize a vessel with a diameter equal to the total size of the aggregate, invalidating the assumption that the present analysis is being done on microvasculature. For these reasons, it was necessary to use a surfactant to reduce the particles affinity for one another and ensure good mixing of the sphere solution. Based on the recommendation of Sphereotech R\&D and on past microsphere work, a solution of approximately $0.01 \%$ Tween ${ }^{\circledR} 20$ was used to suspend the spheres before injection. Tween ${ }^{\circledR} 20$ technical data suggests that a solution of less than $0.05 \%$ is safe (in an acute setting) for mammalian cells (AJH). The solution here is further diluted by a factor of approximately 40 by the animal's blood, calculated as follows:

$$
\begin{gathered}
(0.01 \% \text { Tween } \AA 20)(350 \mu \text { L solution })=0.035 \mu L \text { Tween } \AA 20 \\
\left(\frac{0.035 \mu L \text { Tween } \AA 20}{13 \text { ml blood per animal }}\right)=0.0027 \% \text { Tween } \AA 20 \text { in circulation }
\end{gathered}
$$

Further calculations, including the theoretical ratio of particles to red blood cells, and underlying assumptions are in Appendix II.

\section{Tissue Processing Considerations}

These microparticles are stable for at least one year (manufacturer's recommendation, http://www.spherotech.com/pdetail.html). Refrigeration is not recommended, but the particles have been frozen in tissue for up to 18 months in this lab with no detectable change in 
fluorescence. They are not affected by most stains and dyes. They are degraded by organic solvents, including xylene, DMSO and acetone and are stable in water miscible solvents, including alcohols. Solvent stability is detailed in Appendix I.

To reduce the risk of encountering actual particles with the cryostat blade, it is recommended that sectioning be done at "several times the thickness of the largest spheres" (De Visscher, Haseldonckx et al. 2006). In these studies sections were cut at $90 \mu \mathrm{m}$ thick. It was never apparent that a $10 \mu \mathrm{m}$ sphere had been hit with the blade, but there was one image taken during preliminary pilot testing in which a $22 \mu \mathrm{m}$ sphere was sliced.

\section{MATLAB Analysis}

Protocols for quantification of microspheres have been established in the past and include manual counting of dots and luminometer measurements of chemically digested tissue. Here, the goal of accurately counting FMs in cryosectioned tissue is approached by using an automated analysis program. The open source analysis package "ImageJ" (NIH Image, http://rsbweb.nih.gov/ij/) has been used for this type of work in the past, but, "[ImageJ's] design . .. is geared more towards the analysis of individual images (comparable to Adobe Photoshop) rather than flexible, high-throughput work. Macros can be written in ImageJ for high-throughput work but adapting macros to new projects requires that biologists learn a programming language." (Carpenter, Jones et al. 2006)

The computing environment MATLAB (Mathworks, Natick, Massachusetts, U.S.A.) was chosen for several reasons. It is free to students at this university and most major research institutions and is also a widely used commercial programming tool in industry settings, so its popularity means that online resources and example code are abundant. Bioengineering students study MATLAB for a minimum of two semesters, and some bioengineering faculty are MATLAB coding experts. This software is specifically designed to manipulate matrices and, because images are matrices, MATLAB has excellent image processing capabilities. 


\section{CHAPTER 2: MATERIALS \& METHODS}

This section first describes general animal use, spinal cord injury surgery, and post-operative care, all of which are standard procedures at KSCIRC. Following that is a subsection detailing injection of LEA, a previously documented procedure (Benton, Maddie et al. 2008), and injection of FMs. Both injection of FMs and processing of tissue containing FMs are new techniques in the context of spinal cord research.

Study Design and Experimental Protocol

A total of 21 animals were used. All experiments were done using female, SpragueDawley (SD) rats weighing 190-230 g, except for exploratory surgeries, which were done on two female SD rats weighing approximately $310 \mathrm{~g}$. Experiments were performed in accordance with the guidelines of the University of Louisville Institutional Animal Care and Use Committee. Experimental variations are summarized in Table 5 (Appendix II).

\section{Spinal Cord Injury Surgery}

Animals were sedated to surgical plane with an intraperitoneal injection of 5\% pentabarbitol $(60 \mathrm{mg} / \mathrm{kg})$. Extra pentabarbitol solution was used to boost the original dose as needed during surgery, in $0.1 \mathrm{cc}$ increments. Sedative procedures were the same for SCI procedures and FM/LEA injection procedures.

Spinal injuries were done on 6 animals. Injury consisted of a dorsal midline incision over the midthoracic spinal region, single-level laminectomy at the T12 vertebra, and a controlled contusion injury using the Infinite Horizons (IH) Ipactor (Infinite Horizons Inc., Lexingon, KY) 
at the T9 spinal level (vertebral and spinal levels are anatomically mismatched by three levels at this point). The dura mater is left intact when using this impactor and the spine is stablized using two pairs of forceps, anchored to adjacent intact vertebre. The IH Impactor delivers a forcedefined impact to the spinal cord and has been shown to be a repeatable and reliable way to mimic human contusion injuries in a rodent model (Zhang, Burke et al. 2008). The IH Impactor was set to 150 kilodynes of impact force for all SCI surgeries in this study; this is considered a mild injury.

The wound was immediately closed in layers. Musculature was sutured internally and skin was closed with surgical staples; topical antibiotic (Bacitracin) was applied to the entire incision area. Animals undergoing surgery received $5 \mathrm{~mL}$ bolus s.c. injection of saline during surgery and $5 \mathrm{~mL}$ again afterwards to prevent perioperative dehydration. Once it was apparent that anesthesia was wearing off and the rats were healthy after surgery, they were returned to their cages. Animals were doubly housed, food and water were available ad libitum and one side of each cage was placed on a warm heating pad overnight following surgery. Bladder expression was performed twice daily for 1 day, at which point animals were able to void spontaniously. All spinally injured animals were given gentomycin sulfate $(15 \mathrm{mg} / \mathrm{kg})$ prophylactically, to prevent infection, on post-surgery days 1,3 , and 5 as well as buprenex for pain control $(15 \mathrm{mg} / \mathrm{kg})$ on days 1 and 2 .

\section{LEA Injections}

In a surgery separate from the spinal injury surgeries, thirteen animals were injected with LEA either 7 days post injury (6 animals) or as uninjured control subjects ( 7 animals). Animals were deeply anesthetized and $0.1 \mathrm{~mL}$ volume of $100 \mu \mathrm{g} / \mu 1$ FITC-conjugated LEA solution (Vector Laboratories, Burlingame, CA) was injected into an exposed external jugular vein using a syringe pump set at $1 \mathrm{ml} / \mathrm{min}$. Right or left jugular veins were accessed without preference for a side. After injection, the animal remained sedated while LEA circulated at least 15 minutes and 
no longer than 20 minutes. After this point, it was affirmed that the animal had a strong heartbeat and was breathing normally before FM injections were begun.

\section{FM Injection Preparation}

Original microsphere solutions from the manufacturer consisted of particles, DI water, and sodium azide. Each vial was thoroughly vortexed, according to the manufacturer's recommendations, before aliquoting. To achieve a final sphere count for injetion of about $22 \times 10^{6}$ (1.8 $\mu \mathrm{m}$ diameter, red) spheres, $50 \mu \mathrm{L}$ of solution was pipetted from the vortexed solution into a separate tube. A final count of $6 \times 10^{6}(10.2 \mu \mathrm{m}$ diameter, blue) spheres required a starting volume of $200 \mu \mathrm{L}$ from the original solution. Aliquotted tubes were centrifuged at $2000 \mathrm{rpm}$ for 2 minutes. Surfactant was discarded and the pellet, containing the microspheres, was reconstituted with $0.01 \%$ Tween ${ }^{\circledR} 20$. Reconstituded volumes ranged from $150-200 \mu \mathrm{L}$ depending on the original volume and on the exact surgical protocol.

Tween ${ }^{\circledR} 20$ solution was used because the slight negative surface charge on each particle increases the particle's affinity for one another, increasing the liklihood that they will clump together when suspended in pure dionized water. The manufacturer recommended using a surfactant (specifically Tween 20 or Tween 40) to increase random particle dispersion. Tween 20 was prepared as a $0.01 \%$ solution, according to FM manufacturer's suggestion and data on safe concentrations of Tween 20 (AJH), and this solution was used to resuspend the particles after centrifugation.

\section{FM Injections}

To develop an ideal surgical technique, FM injections were approached in three different ways: left ventricular injections (unventilated), descending aortic injection, and ventilated left ventricular injection. All FMs had a tendency to settle out of solution over time, so they were kept in vials and vortexed in the surgery room immediately prior to being drawn up in syringes 
for injection. Filled syringes of FMs were also vortexed if they were not injected within 1 minute of being drawn. Insulin syringes were used instead of typical 1cc syringes because normal syringes were found to have a dead space of approximately $0.1 \mathrm{~mL}$. Since sphere solutions were prepared on a per-animal basis prior to being drawn into each syringe, and since the volume of each injection was less than or equal to $0.2 \mathrm{~mL}$, the $0.1 \mathrm{~mL}$ of lost volume introduced too much error and made it impossible to predict the final number of spheres injected into each animal. Insulin syringes have no deadspace (other than the volume of the injection needle).

For the first series of pilot surgeries, microspheres were prepared as described and were administered as a bolus injection into the left ventricle to either uninjured animals or to animals on Day 7 post-SCI. When two or more FM sizes were used, the smaller were always injected first to reduce the likelihood that smaller particles would back up behind larger ones as they embolized microvessels. During initial surgeries, animals were not put on respirators. Since the thoracic cavity was no longer able to generate the negative pressure required for inspiration, animals survived roughly two minutes from the time the sphere injection surgery was begun.

Since animals died very quickly upon thoracotomy, injecting FM solutions into a major artery outside the cavity would enable animals to complete a greater number of normal cardiac cycles after FM injection before their bodies started to reduce blood flow to organs and tissues. A series of individual animal pilot surgeries were done in which the descending aorta was exposed via a ventral abdominal incision and gentle displacement of intraperitoneal organs. A catheter was placed in the descending aorta, just rostral to the renal arteries, and FMs were injected as a bolus and allowed to circulate for several minutes. All animals studied in these pilot surgeries were without spinal injury or other complication.

The final iteration of FM injection techniques was done using 6 anesthetized animals (3 were 7 days post SCI and 3 were uninjured controls). Each was injected with LEA in a jugular vein and, after at least 15 minutes of LEA circulation, was intubated via tracheotomy and a mechanical ventilator was used to sustain breathing for the duration of FM injection. Isofluorane 
gas (2\%-5\% variable doses) was used to maintain surgical plane anesthesia while a thoracotomy was done to access the heart. Two different solutions of FM's were injected slowly into the left ventricle, smaller FM's first. Injections were at least 45 seconds apart to optimize random distribution of all particles throughout tissue. After at least two minutes had passed since the second FM injection, and while still under deep anesthesia, animals were disconnected from the ventilator and allowed to expire.

\section{Tissue Processing \& Histological Analysis}

Once the heart was no longer beating, organ biopsies were taken, stored in $4 \%$ paraformaldehyde (PFA) and refrigerated. Tissues included lung (lower right lobe), kidney (left organ), hindlimb muscle (left gastrocnemius), cardiac muscle (heart apex), and liver (upper left lobe). The entire spinal column, including the spinal cord plus vertebral bodies, spanning from the brain stem to the cauda equina, was removed and also stored in 4\% PFA and refrigerated. After 24 hours, PFA solution was discarded and replaced with fresh 4\% solution. Note that these were fresh dissections and did not involve transcardial perfusion with saline or any fixative.

After a total of three days in PFA the spinal cord was dissected and the bony vertebral column remains discarded. The organ samples and spinal cord were submerged in fresh PFA for 24 hours and then transferred to $30 \%$ sucrose with sodium azide for at least four days before being blocked. Sucrose solution was exchanged for fresh stock after the first two days.

Spinal cords were cut into four pieces of approximately equal length and blocked in mounting media (TFM, Triangle Biomedical Sciences, Durham, NC), then frozen at $-40^{\circ} \mathrm{C}$. Blocked tissue was sagittally sectioned on a cryostat at $90 \mu \mathrm{m}$ thickness. Sections were thaw mounted on charged microscope slides and stored at $-40^{\circ} \mathrm{C}$. Prior to coverslipping, mounting media was peeled away with forceps. Fluoromount was used to coverslip slides. To see if tissue and/or FMs had accidentally been peeled away along with the fluoromount, the dried fluormount was collected as slides were prepared and it was examined under the microscope. Only dura 
matter and occasional fragments of ganglia were seen, so it can be assumed that neither spinal tissue nor FMs were removed along with the media during this step.

All micrographs were taken using a Spot CCD camera and capture software (Diagnostic Instruments Inc., Sterling Heights, MI, USA). Exposure times, gain, and magnification were adjusted for different fluorophores but kept constant for the same fluorophores across tissue sets.

\section{MATLAB Code}

The MATLAB code serves to automate fluorescent microsphere detection. It has two primary functions in the analysis: counting total spheres detected per image, and accumulating a pattern in sphere distribution among cumulative images.

The most important function of the MATLAB sphere detection program is referred to in text and in the code as "region growing". Prior to referencing this function, the main program first reads the image(s) set by the user and converts the color micrographs to black and white images. The images are not converted to grayscale, which would include shades between black and white, but rather to binary images of only black pixels and white pixels. The conversion is based on an intensity threshold which can be adjusted by the user. All white pixels in the binary image are read by the Region Growing function; its input is a series of coordinates and whether the coordinate is black or white. It detects "regions" (which we interpret here as fluorescent spheres) by identifying the very first white pixel in the image. Determination of the "first" white pixel is based on 2D coordinates of each pixel. Starting with the coordinate of the first white pixel, Region Growing checks every pixel adjacent to the first one to see if the adjacent pixels are white, too. If one or more are white, it repeats the check over and over for each of those white pixels. It checks in all directions finding new white pixels until the region is fully "grown", or surrounded by black pixels. When it finds that there is no other adjacent white pixel, it stops checking. Thus, when Region Growing is run once, its output is a list of pixels it has identified as comprising one continuous region. 
So far, the code has only identified one region. To identify another, it subtracts the first Region Growing output (a list of coordinates referencing each pixel in the first detected region) from the previous input (a list of coordinates referencing all white pixels in the whole image) so the new input is a list of all white coordinates except the ones already identified. Now when the function runs, it again detects the first white pixel, but it is not the same one it detected in the previous loop. Region Growing continues to identify the first white pixel, then all adjacent white pixels, then subtract those until there are no more coordinates left. Once it cannot identify any more white pixels at all in the image, it exits that loop. The output is a series of uniquely identified regions of coordinates. This output is used in the main code.

The main code averages all $\mathrm{x}$-coordinates associated with the white pixels in one region and all y-coordinates associated with the white pixels. This simplifies the region so it is now identified by its centroid. The total number of centroids is used to make the bar graphs and line plots representing total sphere count per image. The color intensity plots are made by defining the bin size (how large or small the colored squares shall be) and adding a value of 1 to any bin in which a centroid coordinate is detected. Color gradients are automatically scaled according to the range of data being presented. Titles are added to the plots which automatically display pertinent user-defined variables, including binary threshold, number of images averaged, and which images were analyzed and displayed in the figure.

It is possible to simplify the code such that centroids would be detected directly from grayscale images, rather than running the Region Growing function from internally generated binary ones. This would provide equal data output to the user, but it would eliminate useful finetuning features inherent to the Region Growing code. For example, a user would not be able to implement more accurate counting algorithms to count large regions, which in reality are comprised of two or more spheres, as a true count. One region would always be limited to a count of one. Enormous fluorescent debris in the micrograph or background noise registered as a one-pixel-region could likewise not be eliminated from the count if area centroids were detected 
directly. These functionalities are only possible if a theory like the Region Growing code is used for analysis.

An algorithm was included in the main code, after Region Growing, which automatically averages sphere counts for a user-defined number of consecutive images. The average is calculated as a true mean rather than a moving average because the goal was to reduce the complexity of the data for easier user interpretation, rather than simply reducing the amount of apparent variation in the display. The average is accomplished using the mod command to compare the user-defined "number of images to average" (an integer, "X") with the number of images analyzed so far. Mod calculates the remainder of an arithmetic division. When the number of images analyzed so far divides evenly into the number " $\mathrm{X}$ ", $\bmod$ is equal to exactly zero so the program calculates the average of the previous " $\mathrm{X}$ " images. When it does not divide evenly, mod is not equal to zero, so the code continues to analyze images until it finds mod equal to exactly zero again. Total sphere count per image is displayed as a bar graph; average count over " $\mathrm{X}$ " images is displayed as a dot plot.

MATLAB, by nature, can be re-written at any line. The features summarized in Table 2 are designed to be robust under the entire range of adjustment settings listed. These features are labeled with comments and the most frequently adjusted features have a row of comment symbols above to make them easier to find. These are the features a user is most likely to adjust in order to obtain the most useful results.

\begin{tabular}{|l|l|}
\hline \multicolumn{1}{|c|}{ Adjustable Feature: } & \multicolumn{1}{c|}{ Range or Settings } \\
\hline Threshold & $1-255$ \\
\hline Images to process & A series of consecutive indices, any length \\
\hline Relevant parameter & "Total FM count" or "FM distribution" \\
\hline Number to average (averaging bin size) & $1-$ [total number of images analyzed] \\
\hline Colormap bin size & $1-$ [dimension of image], 30 - 100 are shown, Fig. 33 \\
\hline
\end{tabular}

Table 2: MATLAB features which are conveniently adjusted by the user. 


\section{CHAPTER 3: RESULTS}

Accuracy of MATLAB sphere identification

The MATLAB program for counting total FMs per image was run on a sample of 20 specific images. These 20 images were selected to span the range of image conditions seen within the overall image set and included images from every animal. Variability accounted for in the 20 sample images included image focus (crisp, average, and poor), background fluorescence (low and high), debris in the image (present or lacking) and co-occurrence of spheres (clumped particles and individual particles). Statistical methods were then used to determine whether any of these factors effects the accuracy of MATLAB FM counting compared to human counting.

To find the number of FMs a human identifies in each image, images were randomly renamed with numbers 1-20 to eliminate possible human biases caused by assuming images from the same animal would be similar. Three lab members manually counted the spheres they saw by opening each image in ImageJ and using the counting tool to identify spheres one at a time. They recorded the number of spheres they actually counted, the number of spheres they suspected the tissue truly contained (useful when a region appeared to contain multiple spheres indistinguishably clumped together or when a fluorescing particle was suspected to not actually be a sphere) as well as the number of minutes they spent on the task. Average manual counts and the standard deviation of the manual counts were calculated. This was compared to MATLAB counts of the same images with the MATLAB threshold set at 180 and also at 210.

Furthermore, the corresponding 20 tissue sections were examined under the microscope for the most precise FM counting possible. Microscope counting was most precise because when spheres were so close together that they were indistinguishable using the $4 \mathrm{x}$ objective, magnification could be increased to $10 x$ or $20 x$. Sometimes it was still impossible to see the 
particles individually, but it was possible to focus first on one sphere and then see the other sphere(s) come into sharper view as the focus was adjusted over a range. Microscope counting should be considered separately and not compared to other counting methods because the MATLAB program and lab members both used images collected with the lower magnification objective. Therefore the outcomes being considered here are not equal and statistical comparisons are not valid.

Data presented in Figure 9 are the average of the three manual counts (with standard deviation bars), MATLAB automated counting at a threshold of 180, and MATLAB counting at a threshold of 210. MATLAB counts are statistically equal to human counts at both thresholds. In the regression analysis presented, $98 \%$ of the variance is accounted for $\left(\mathrm{r}^{2}\right.$ adj $\left.=.98\right), \mathrm{p}<.001$, at $\mathrm{n}=20$. The average standard deviation in human counting is .88 on a range of 50 , which is a $1.6 \%$ standard deviation. Therefore the standard deviation in human counting is comparable to the variance in the error of MATLAB counting. Furthermore, the difference between the two thresholds tests is so small that this sample size (20) does not justify using one threshold compared to the other.

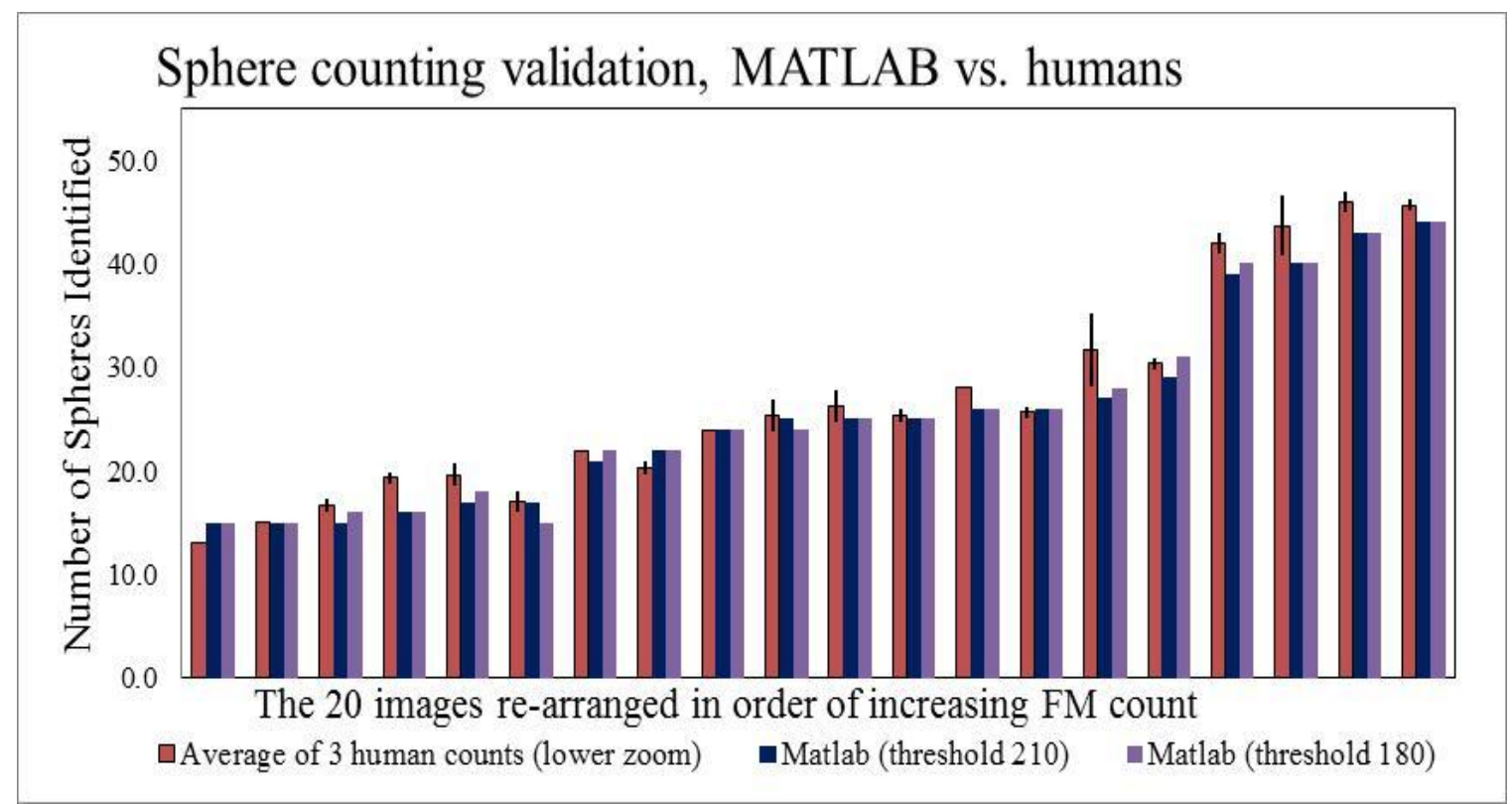

Figure 9: For some images, the lab members counted more FMs than MATLAB. Neither of the two thresholds tested is more accurate in counting FMs in these images. 


\section{Sphere counting validation, comparison to microscope count}

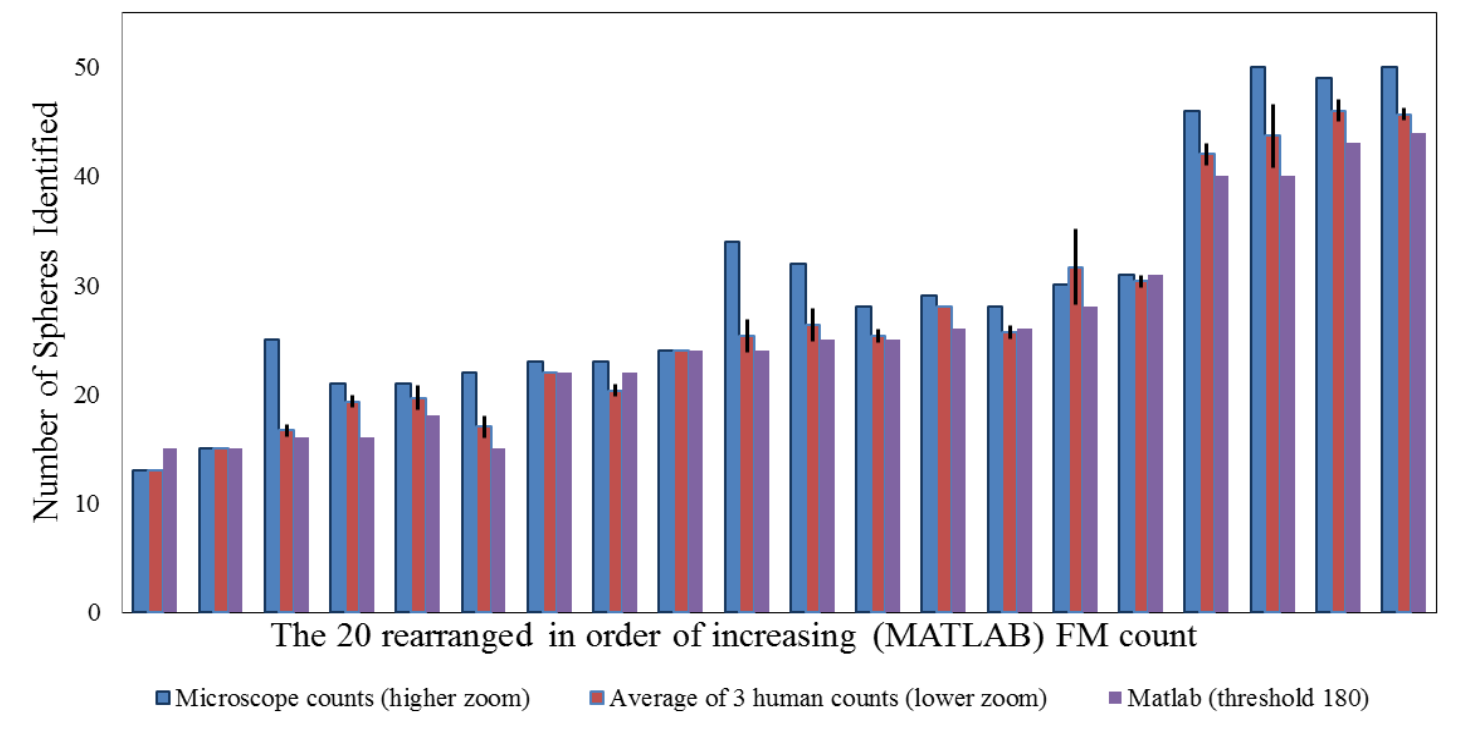

Figure 10: For some images, the "true" count is very close to the measured counts. For a few images, there is a large difference.

Counting the total spheres and writing down the count in 20 images took lab members an average of 14 minutes. MATLAB performs the same analysis, calculates centroids, and produces figures illustrating sphere count and centroid location in 40-41 seconds (data not shown).

Images are graphed here in order of increasing sphere count but this is for visual interpretation and does not indicate a trend in data. There is not a relationship between the number of spheres miscounted and the overall number of spheres truly in the section. Similarly, presence of debris, animal from which the tissue originated, person counting the images, and cooccurrence of FMs are all factors which could theoretically affect the accuracy of MATLAB counting compared to humans, but which it is shown here do not.

Residual plots in Figure 11 show that the 20 images were a good sample because there is no trend in the Versus Fit, Versus Order, or Histogram plots, and there are no data point far from the line on the Normal Probability Plot. 


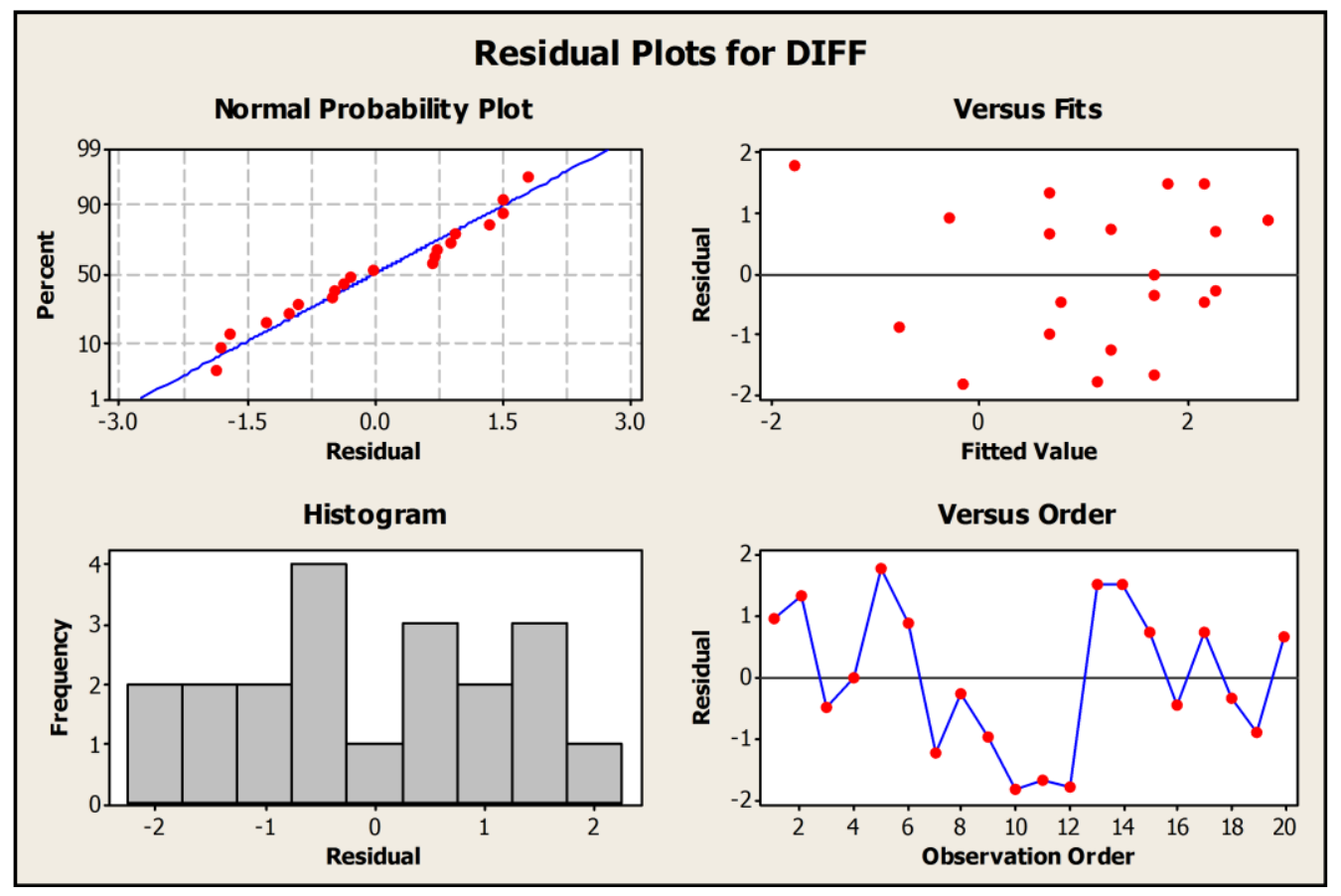

Figure 11: Statistical analysis showing that the difference between MATLAB counting and human counting (DIFF) is not significant and was estimated from images with no apparent inherent bias.

\section{Rostral-Caudal Sphere distribution}

This type of analysis is comparable to luminometer readings, where the total luminosity is directly proportional to the number of FMs in that sample and is indicative of blood supply to that region. Using MATLAB image processing instead of gross tissue digestion enables accurate measurement of one image (one $90 \mu \mathrm{m}$ section) at a time. To do this, the total numbers of "regions" identified by MATLAB in a given image are totaled, and many totals are displayed as a bar graph where each image is represented by one bar. The $\mathrm{x}$-axis is automatically labeled with the image number so if analysis is run on a specific series the axis is labeled with the corresponding image number rather than arbitrarily starting over at zero or one. For bookkeeping reasons, some tissue labels were begun with number 1 and some with 91 but that is not indicative of an anatomical difference. This type of analysis did not require registration and even torn or folded tissue sections could be used, since the total number of FMs per section was the parameter of interest. 
Only the areas of SC tissue containing the injury epicenters (or corresponding levels, for control animals) are studied here. Each frozen block of tissue contained approximately $2 \mathrm{~cm}$ of spinal cord so the data represents the spinal region from roughly T5-L2 (exact level varies by animal). Distances can easily be calculated since each image in the same set is $450 \mu \mathrm{m}$ from the next. Figure 35 is a photo of a spinal cord and provides relative tissue orientation.

For Animal 1, one set of sections (out of 5 sets) was coverslipped and imaged. This means that images saved here represent every fifth section of the spinal cord. Animal 3 was first imaged as one set, and then another set of images was recorded later. It is presented as both single and double sets to compare the level of detail obtained by doubling the image acquisition. Animals 4, 5, and 6, the uninjured controls, are also represented by two of the five sets of sections. Animal 2 is imaged in its entirety, so every single section of tissue in the region of the injury epicenter is analyzed. This means that there are five times as many data points representing the same section of spinal cord for Animal 2 as there are for Animal 1. Comparing the number of images and the outcome of the data is important in planning the tissue and image processing for future studies.

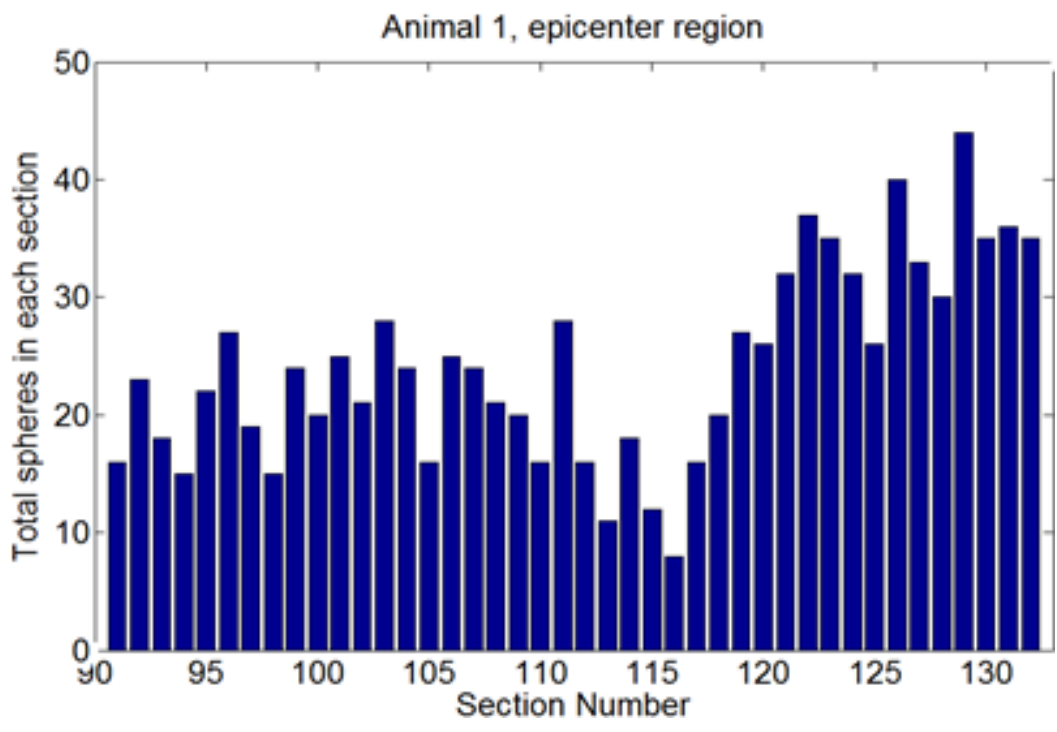

Figure 12: Longitudinal analysis of injured SC, total FMs per section, Animal 1 


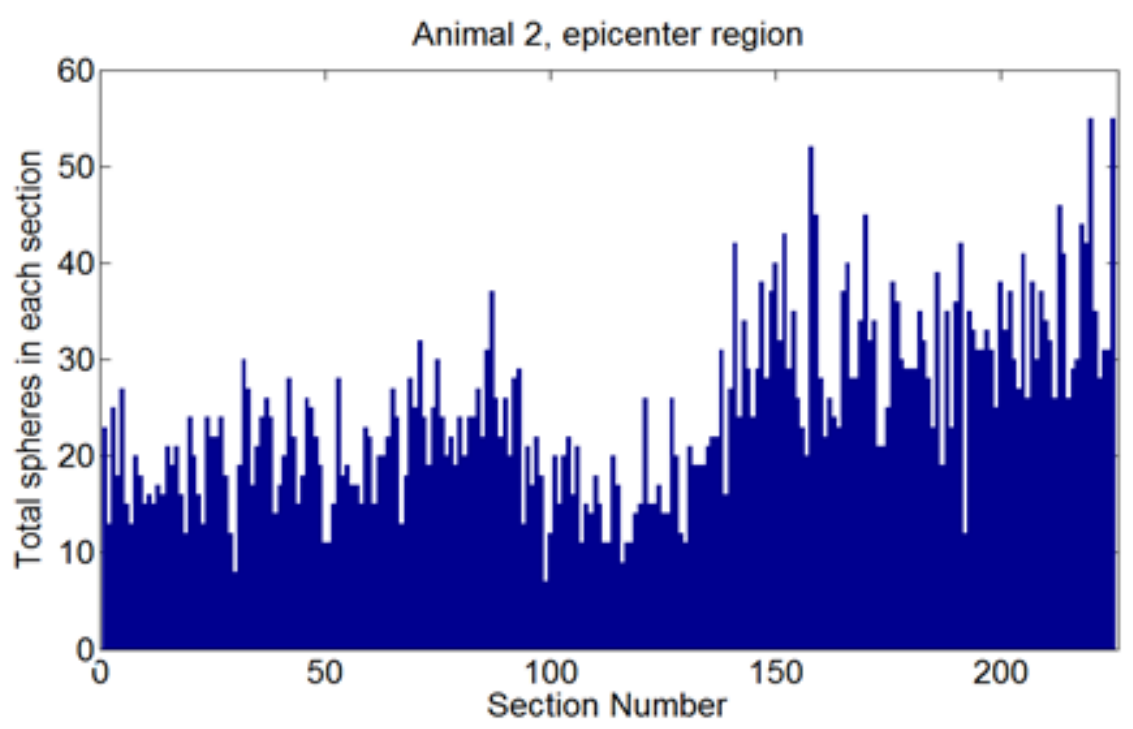

Figure 13: :

Longitudinal analysis of uninjured SC, Animal 4; same number of images as Animal 3; FM count variability is similar in uninjured spinal tissue.

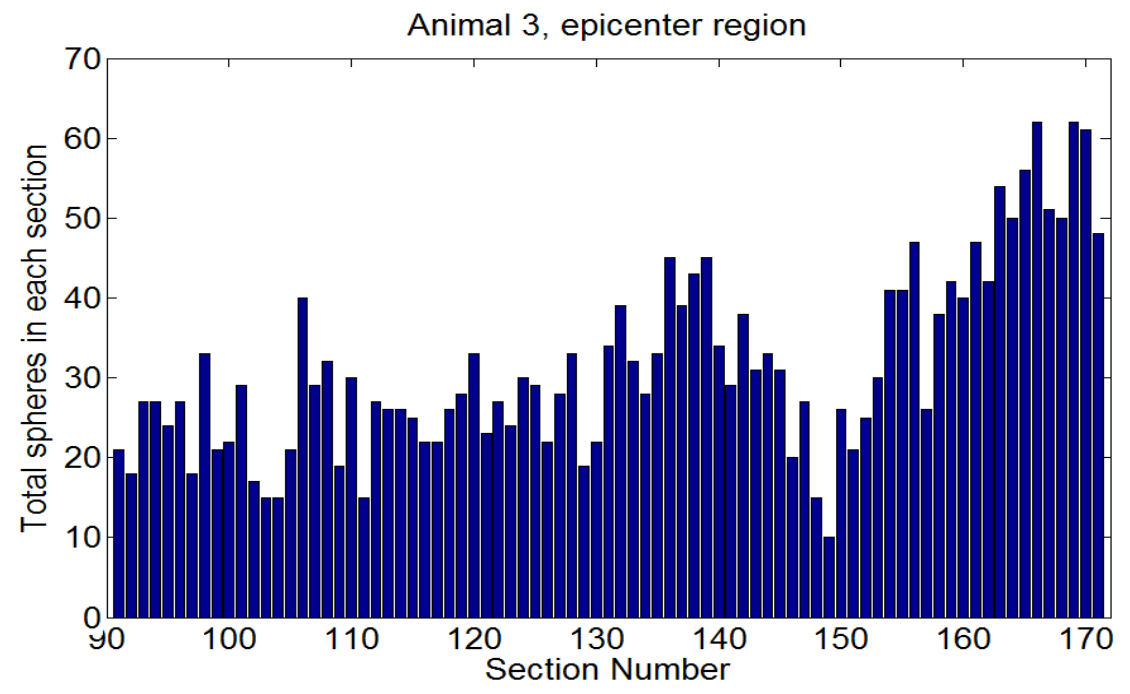

Figure 14:

Longitudinal analysis of injured SC, animal 3; this has twice as many images as Animal 1, but fewer than Animal 2. Notice apparent similarities in the variability.

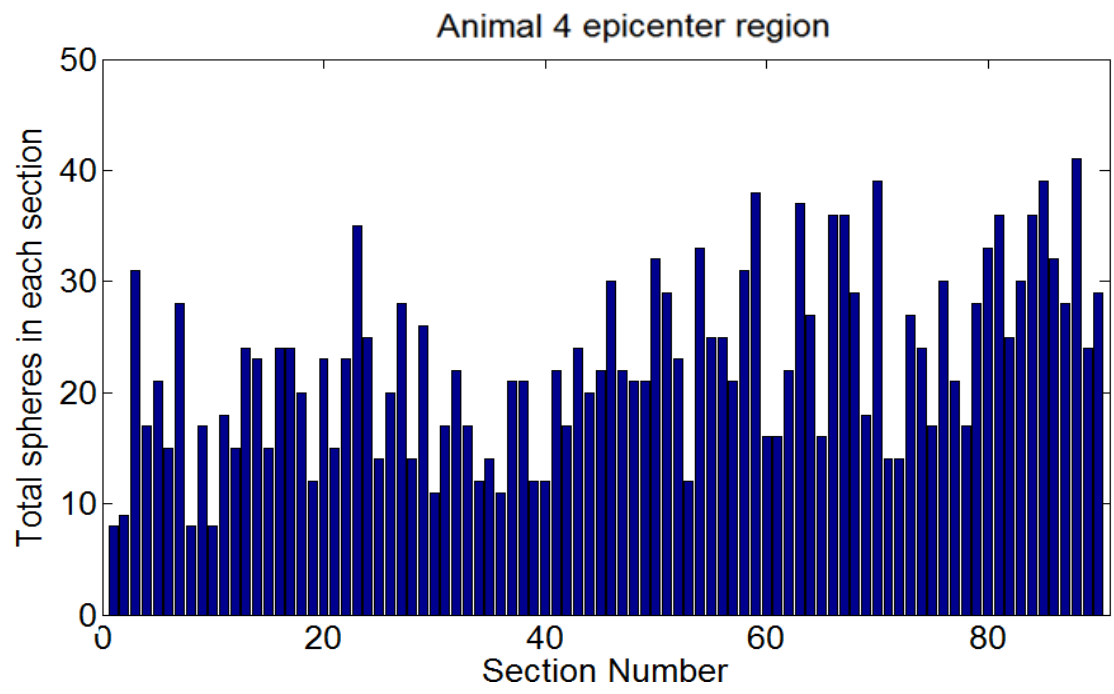

Figure 15: Longitudinal analysis for Animal 4, uninjured 


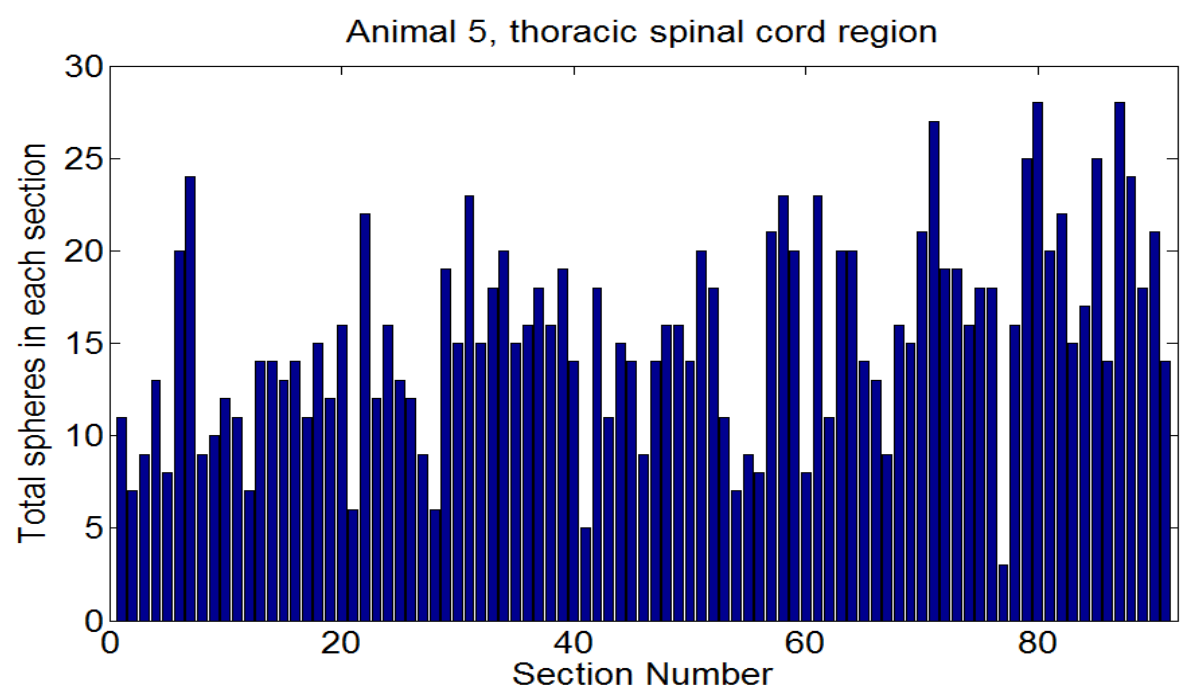

Figure 16:

Longitudinal analysis of uninjured SC, Animal 5,

Animal 6, thoracic spinal cord region

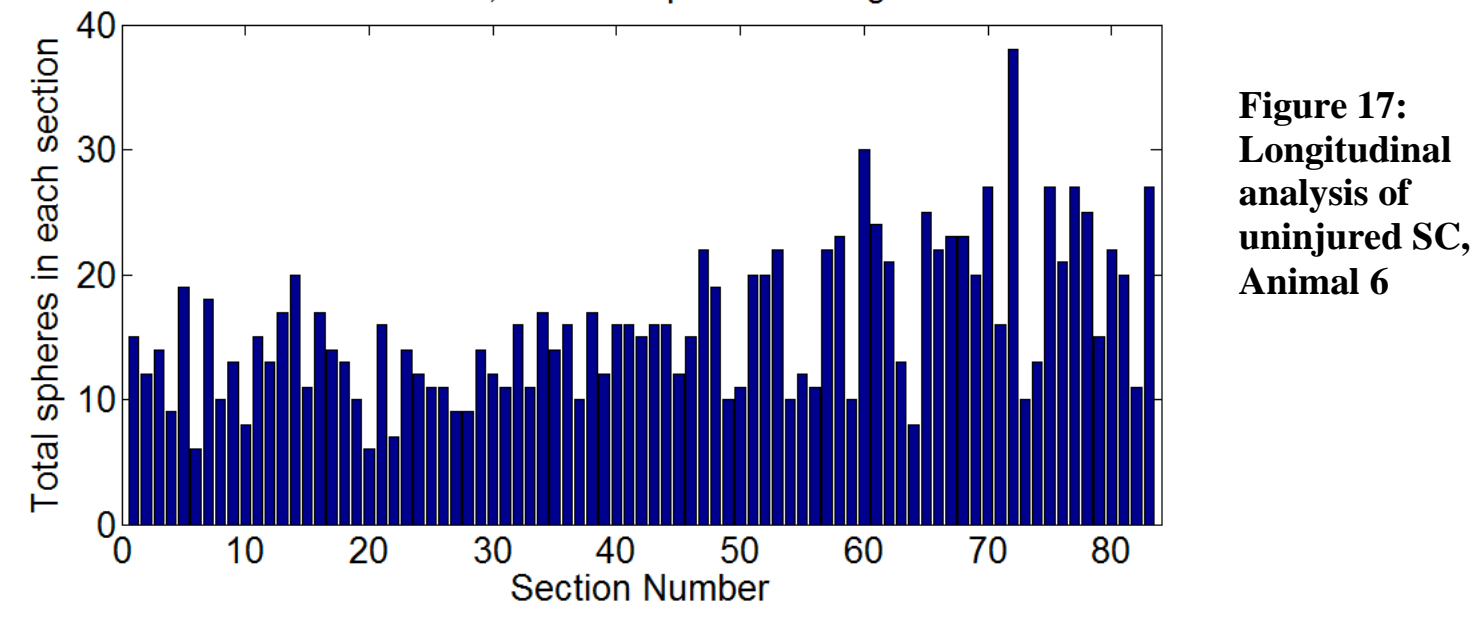

From the bar graphs (Figures 12-17) it is obvious that the data has inherent variability.

Some variability is due to imaging partial tissue sections. The imaging protocol dictated that every single section in a specific set should be recorded even if it was torn or incomplete. Later, a user may decide to ignore such images or replace them with images from another tissue set (another serial set corresponding to the damaged one). Alternatively, a user could use manual or automated tracing of the tissue area to normalize FM count to area. Either option would reduce some of the variability while maintaining the integrity of the data. At this point the data is presented exactly as it was recorded except where a section was missing entirely, in which case the previous image is duplicated and used for both points. Fewer than 1 in 50 images were replaced in this way. 
In order to account for the possibility that human error or cryostat variability led to the variation in FM counts, averaging was incorporated into the MATLAB code. If the variation were a result of the sectioning work there should be a predictable pattern repeated within each set of five images. Averaging the images to one data point should eliminate that fault and the remaining variability should reflect variations in tissue blood supply. Therefore, total sphere counts from sequential images are averaged for every five images from Animal 2, and every two sequential images for Animals 3, 4, 5 \& 6 .

Animal 2, averaged by 5 's
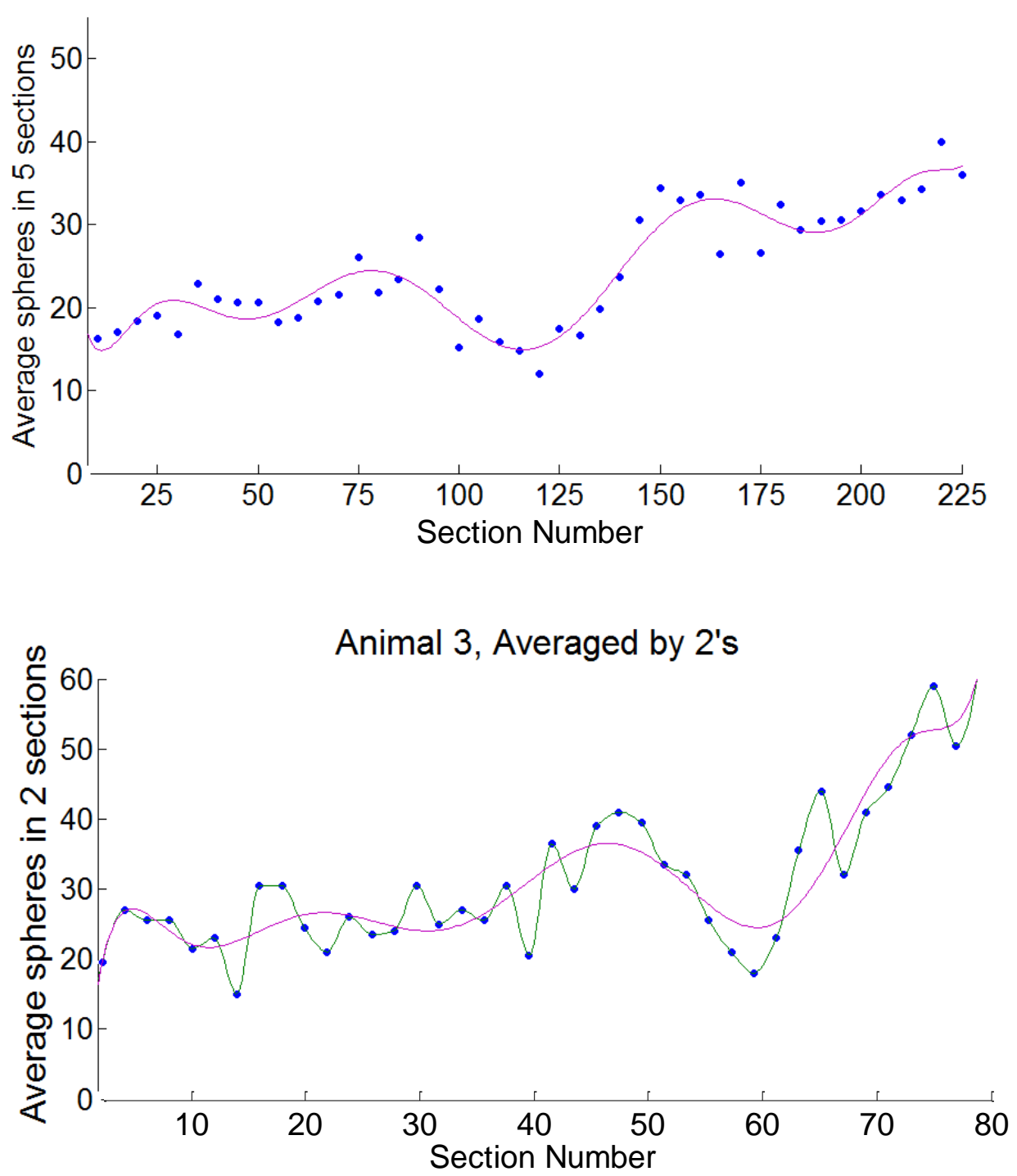

Figure 18:

Longitudinal analysis of injured SC, Animal 2, each 5 consecutive points averaged and a polynomial line added for visual interpretation (not to imply a mathematical relationship)
Figure 19:

Longitudinal analysis of injured SC, Animal 3, each consecutive 2 points averaged; two types of lines added with MATLAB for comparison 
Animal 4, averaged by 2's

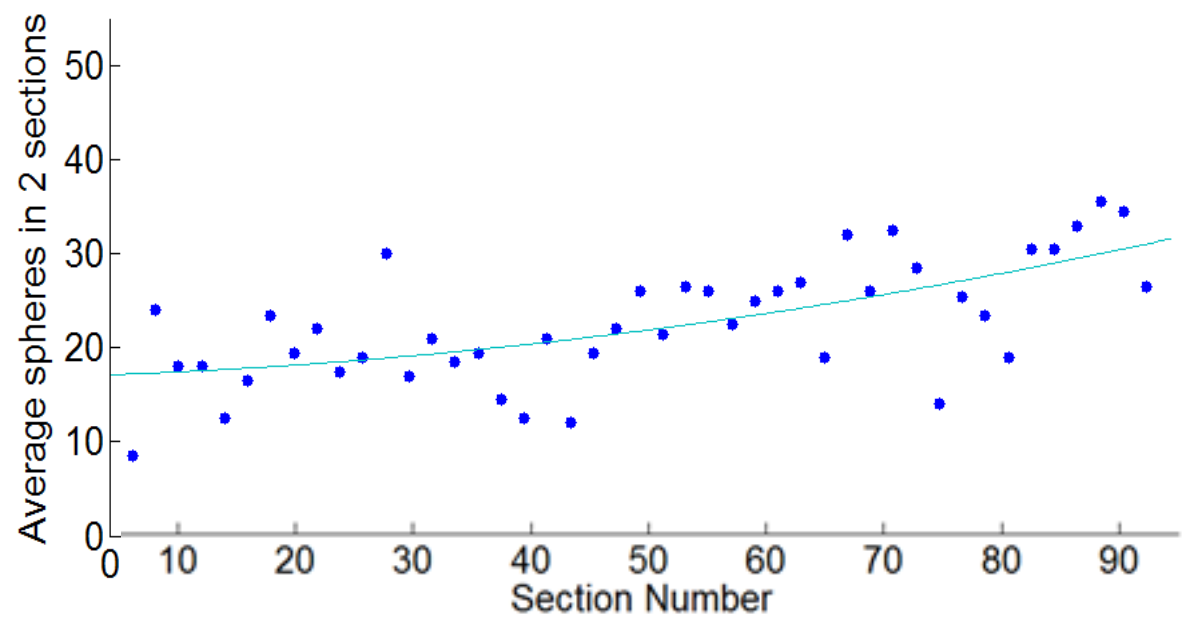

Animal 5 , averaged by 2's

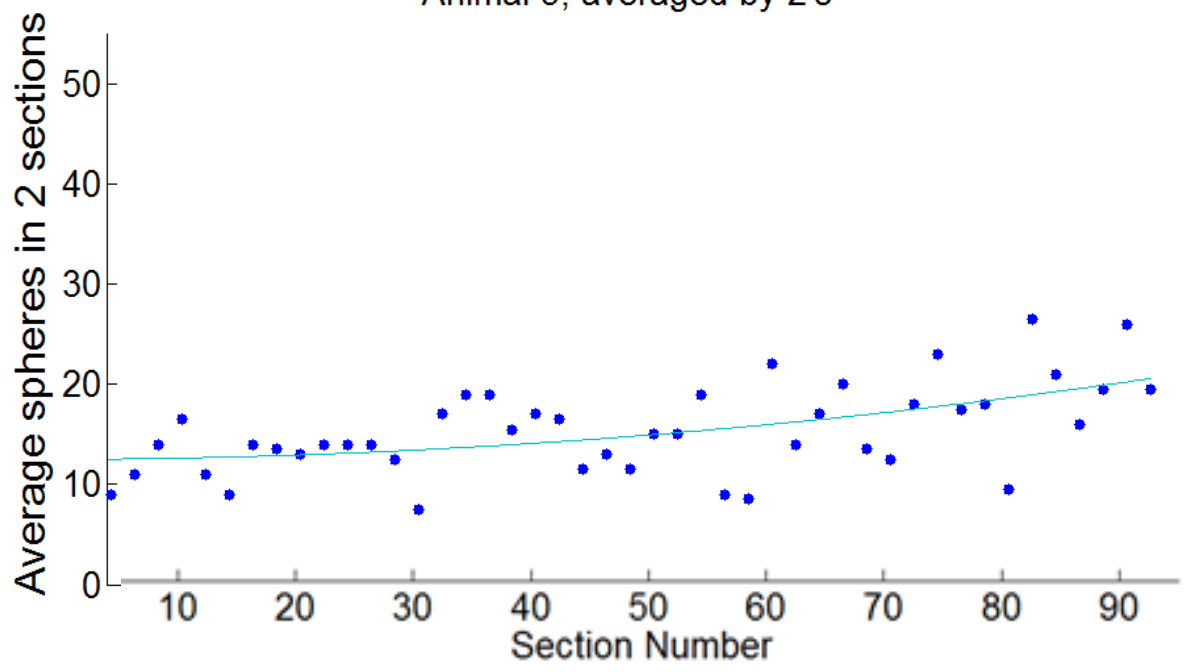

Animal 6 , averaged by 2 's

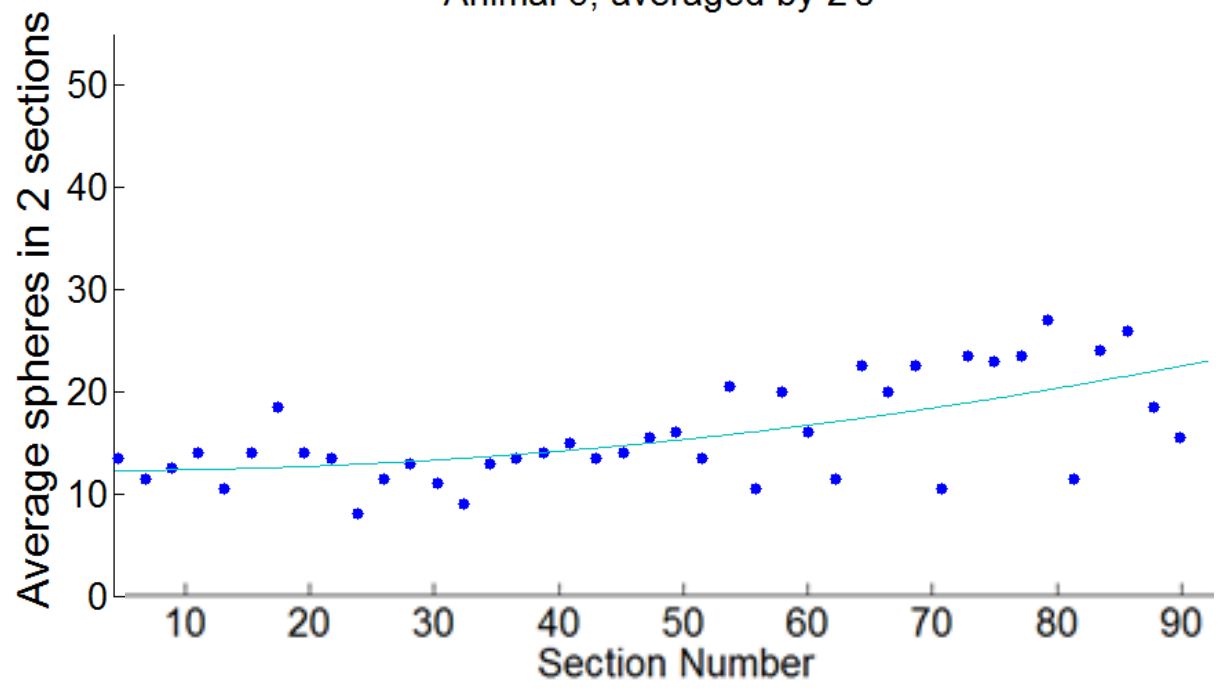

Figure 20:

Longitudinal analysis of uninjured SC

Animal 4, each 2 consecutive images averaged.

Lines of fit are used to calculate $r^{2}$ for comparison of variability in uninjured animals (data not shown).

Figure 21:

Longitudinal analysis of uninjured SC, each 2 consecutive points averaged

Figure 22:

Longitudinal analysis of uninjured $S C$, Animal 6, each 2 consecutive points averaged 
Variation in the averaged data is lower than variation in the original data, reflected by drastically lower $r^{2}$ values, but since this method uses a flat average rather than a moving average the broader trends are not lost. Variation between data sets was not different and this indicates that the variation was not related to whether the first, last, or intermittent section from each set was analyzed.

At this point, the number of total sphere counts averaged (i.e. the number of images summed into each averaging bin) is increased by various increments to illustrate what analysis is helpful in interpreting data. Lines of fit on the following charts, as in the previous ones, are meant to conceptually summarize the points, not indicate a mathematical prediction.
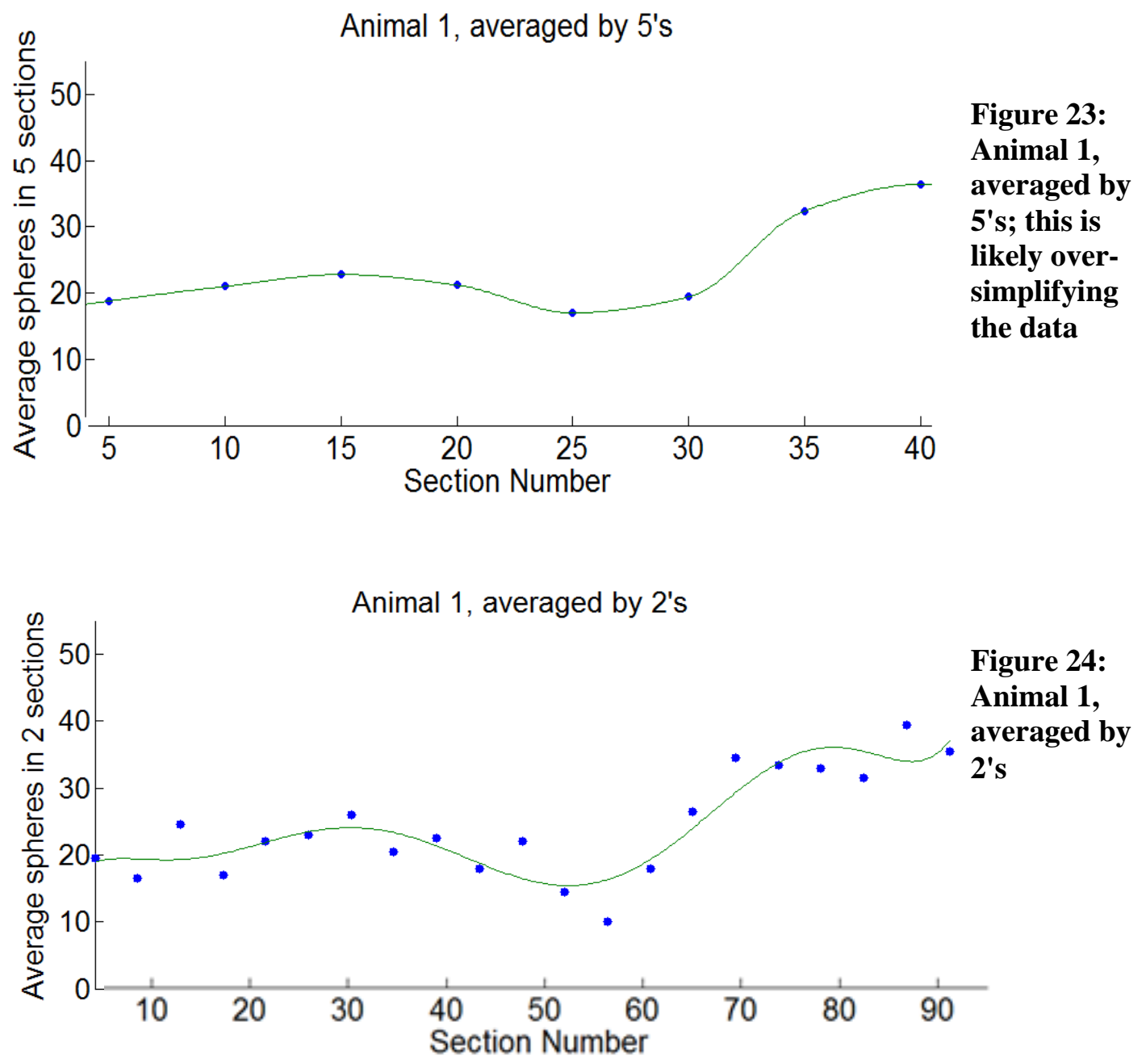
Animal 3 , averaged by 5 's

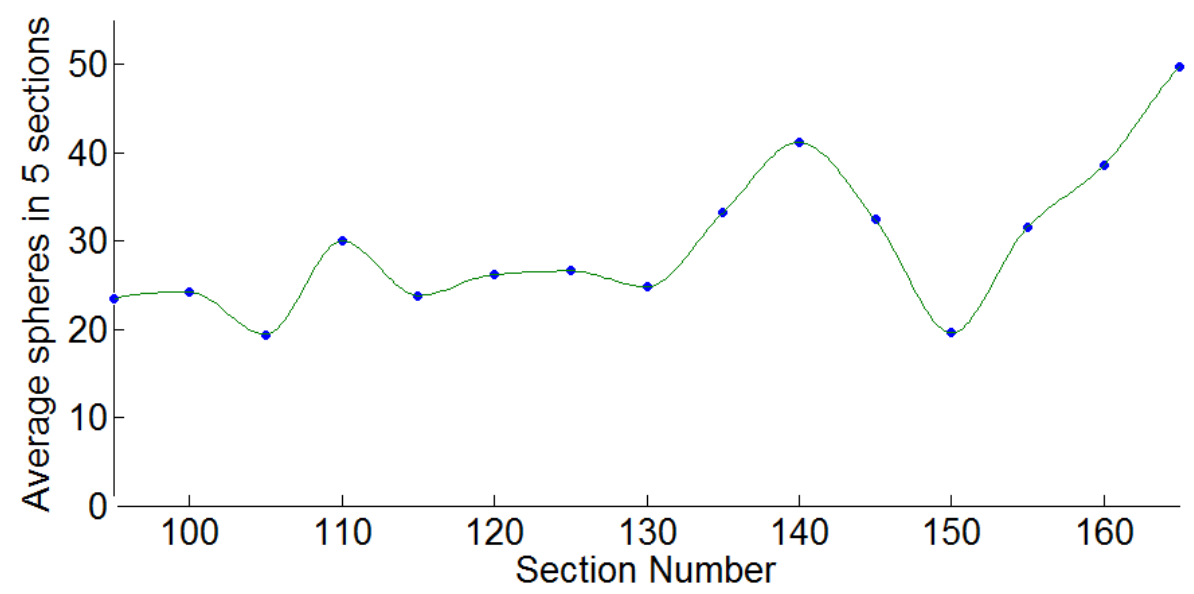

Figure 25:

Animal 2, averaged by 10 's; direct fit line applied, rather than a polynomial

Animal 2, averaged by 10 's

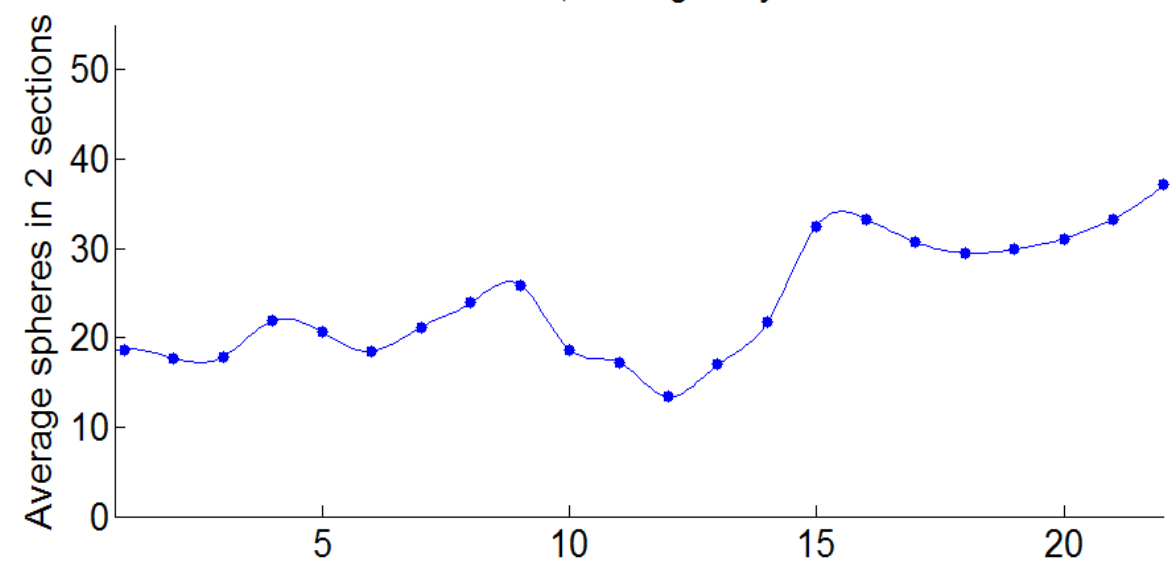

Figure 26:

Animal 3, averaged by 5's

Animal 4, averaged by 5's

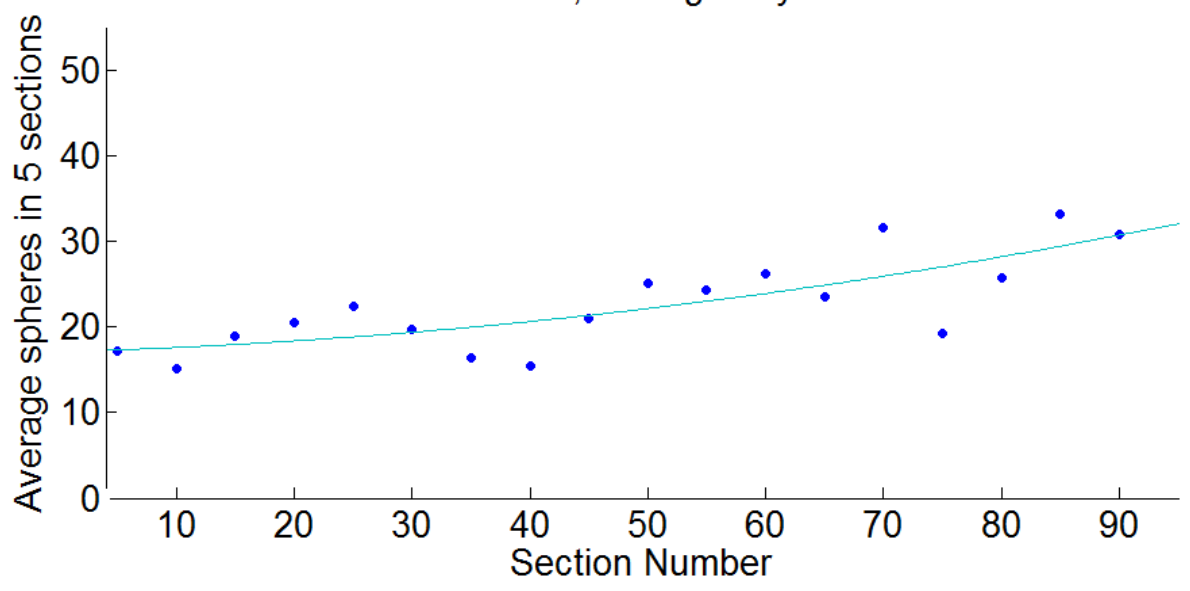

Figure 27:

Animal 4, averaged by 5's 


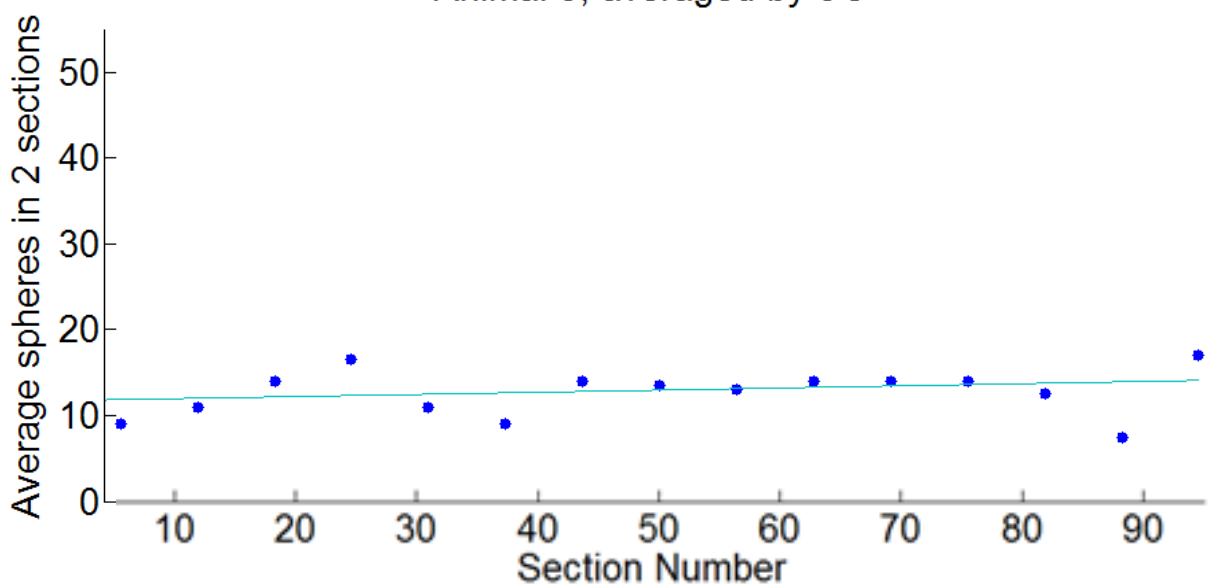

Figure 28:

Animal 5, averaged by 5's

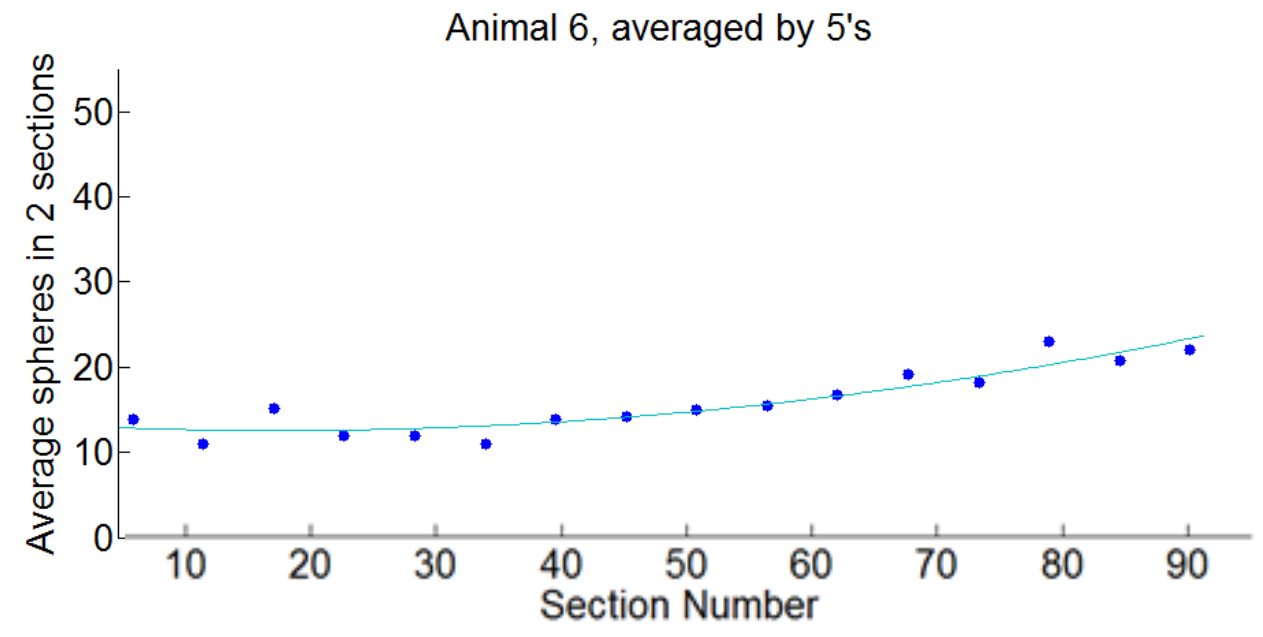

Figure 29: Animal 6, averaged by 5 's

Averaging sequential data points (Figures 18 -29) reduces apparent variability and makes the data easier to interpret. It also preserves the accuracy better than simply lowering the sample size. Different average bin sizes are illustrated and there is clearly not an easy delineation of "reliability" in bin size. Averaging ten images at a time with Animal 2 data reveals a clean summary of the information. Averaging ten images from Animal 1 is an oversimplification. This indicates that the average should be based on true distance rather than number of images. For example, one data point every $900 \mu \mathrm{m}$ results in a representation of the FMs in that region that is sufficient to identify the injury site. At this distance, ten images per average bin from Animal 2 and two images per average bin from Animal 3 represent the same distance so could be considered as equally representative in later calculations. 


\section{Analysis by Spinal Region}

Variation between sets of raw data was comparable so therefore it was valid to do a standard t-test comparing the number of FMs detected. Tissue levels were matched between animals based on grey matter shape and injury location; injury epicenters were aligned and uninjured animals were lined up based on tissue histology. Data from the three injured animals were averaged and compared to averages from the uninjured animals. Aligning the images based on anatomy meant that some of the most rostral and caudal data only existed for two animals rather than all three. It is not proper to derive conclusions from an $n<3$, so therefore these are disregarded any time averaged data is being analyzed. The uninjured and injured data is compared in Figure 30.

In the first $6.3 \mathrm{~mm}$ of spinal level-matched tissue, FM count averaged 22.8 (standard error 0.7, standard deviation 2.0) for injured tissue and 14.6 (S.E. .7, S.D. 2.0) for uninjured tissue. (This calculation was done using 14 measurements from each of the three animal, so comparing groups of 42 calls for standard error calculation rather than standard deviation.) At the injury epicenter, an average of 17.6 (S.D. 1.6) FMs were counted compared to 21.0 (S.D. 1.7) in the same tissue level of control animals. This measurement was done using a smaller sample (5 data points) since the epicenter area is small. The tissue on which this calculation is based spans $1.8 \mathrm{~mm}$. Rostral to the epicenter, the difference was even greater: 38.6 (S.D. 4.1) for injured animals compared to 21.5 (S.D. 2.6) for uninjured animals. See Table 3 for a summary and Figure 30 for graphical display.

Table 3: Comparison of FM distribution in injured and uninjured animals, by region

\begin{tabular}{|l|l|l|}
\hline Region (rel. length) & Injured Average (St. Dev.) & Uninjured Average (St. Dev.) \\
\hline Rostral (14) & $22.1(2.0)$ & $15.6(2.0)$ \\
\hline Epicenter (5) & $17.6(1.6)$ & $21.0(1.7)$ \\
\hline Caudal (7) & $38.6(4.1)$ & $21.5(2.6)$ \\
\hline
\end{tabular}




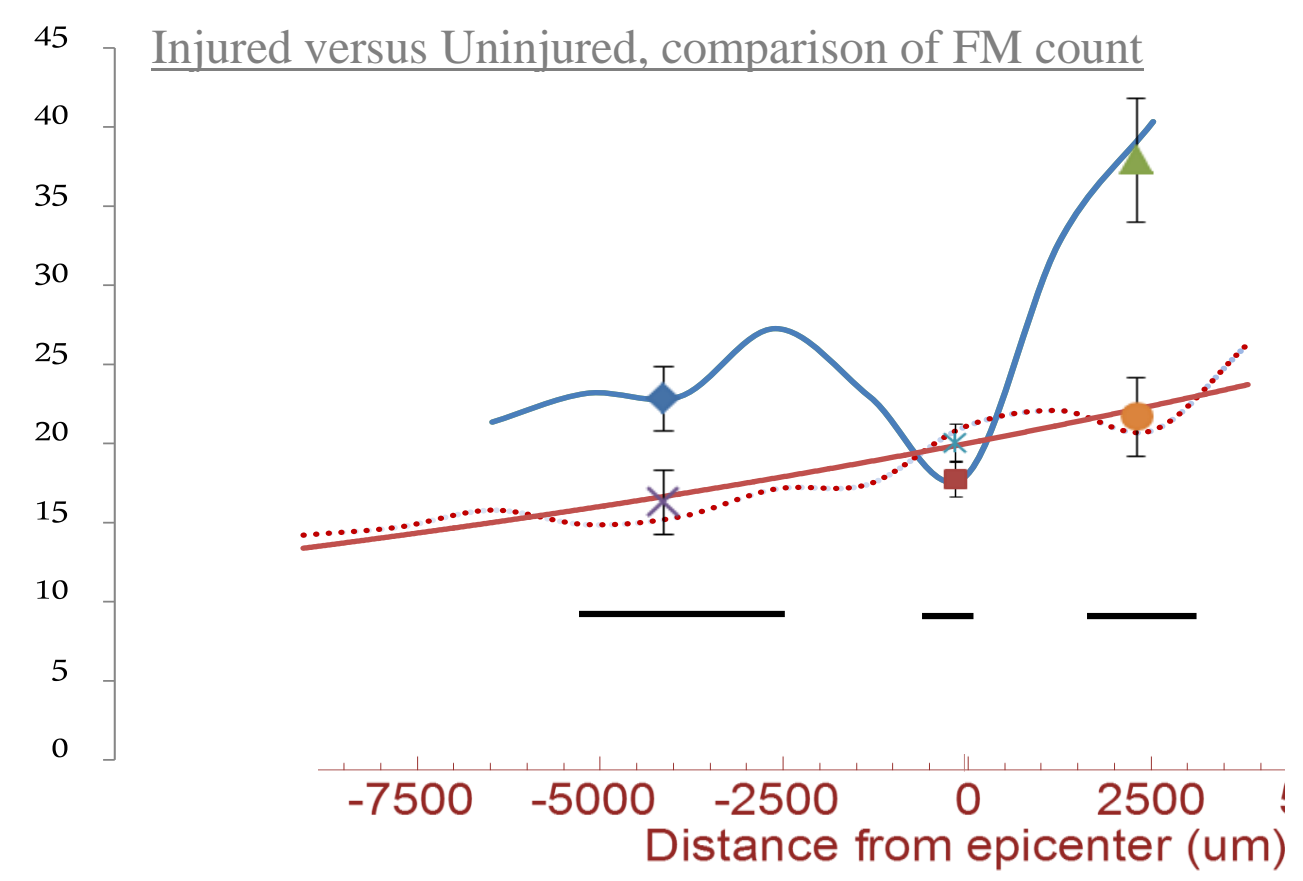

Figure 30: Injured animal data (blue) compared to uninjured trend (red). Y-axis reflects average FM count; $x$-axis corresponds to distance in $4 \mathbf{~ m m}$ increments.

\section{Cross Sectional Sphere Distribution}

This analysis technique presented more variables than the total-sphere-count discussed previously. Adjustments included bin size, threshold, and number of images analyzed per run. Here, bin size refers to how large or small the grid blocks are. Bin size is controlled by adjusting a variable ("grid size") which is the number by which the image's dimensions are divided, to form squares of equal size. A high number results in tinier bins, or smaller grid squares, because the image's length and width are being divided by a larger number. Bin size does not change the actual data but it is an important parameter of the output because appearance of the result is affected by adjusting it. 


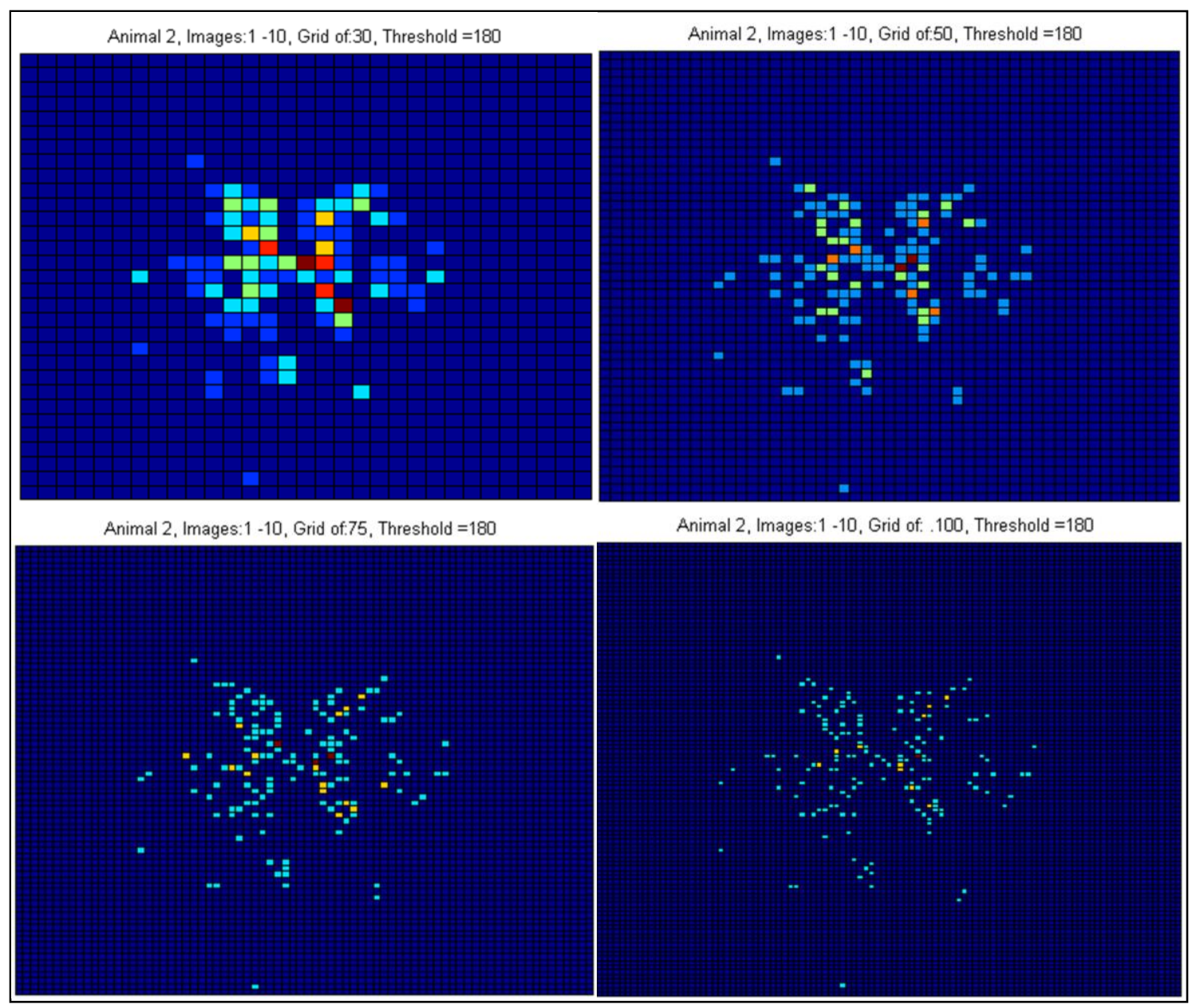

Figure 31: The same 10 images are analyzed using different colormap bin sizes. The information being presented is exactly the same, but it appears different and different assumptions might be made based on how the colormap appears. As the bins get smaller, it is increasingly likely that only one or two FMs will be detected per bin, so the distribution (blood supply) seems less intense.

This is shown to illustrate the importance of choosing an appropriate bin size. The grid should be large enough that multiple FMs are detected in many of the bins, yet not so large that the size of the squares hides information about the distribution pattern.

Figure 32 (next 3 pages): Animal 4 (left side) is an uninjured control and Animal 2( right side) is an injured animal. This figure displays level-matched color plots of corresponding tissue from each animal. Animal 4 was imaged as 2 of the 5 sets, while Animal 2 was imaged in its entirety; therefore the image numbers printed above each colormap correspond but do not match precisely. While there are 2.5 times more images for Animal 2, this does not explain the increased number of FMs detected because the colors on the plot are scale automatically. Apparent blood flow patterns are not affected by the different numbers of images analyzed.

The Figure is a series of 9 sequential colormap (rostral to caudal) followed by 1 cumulative colormap which is outlined in yellow. 


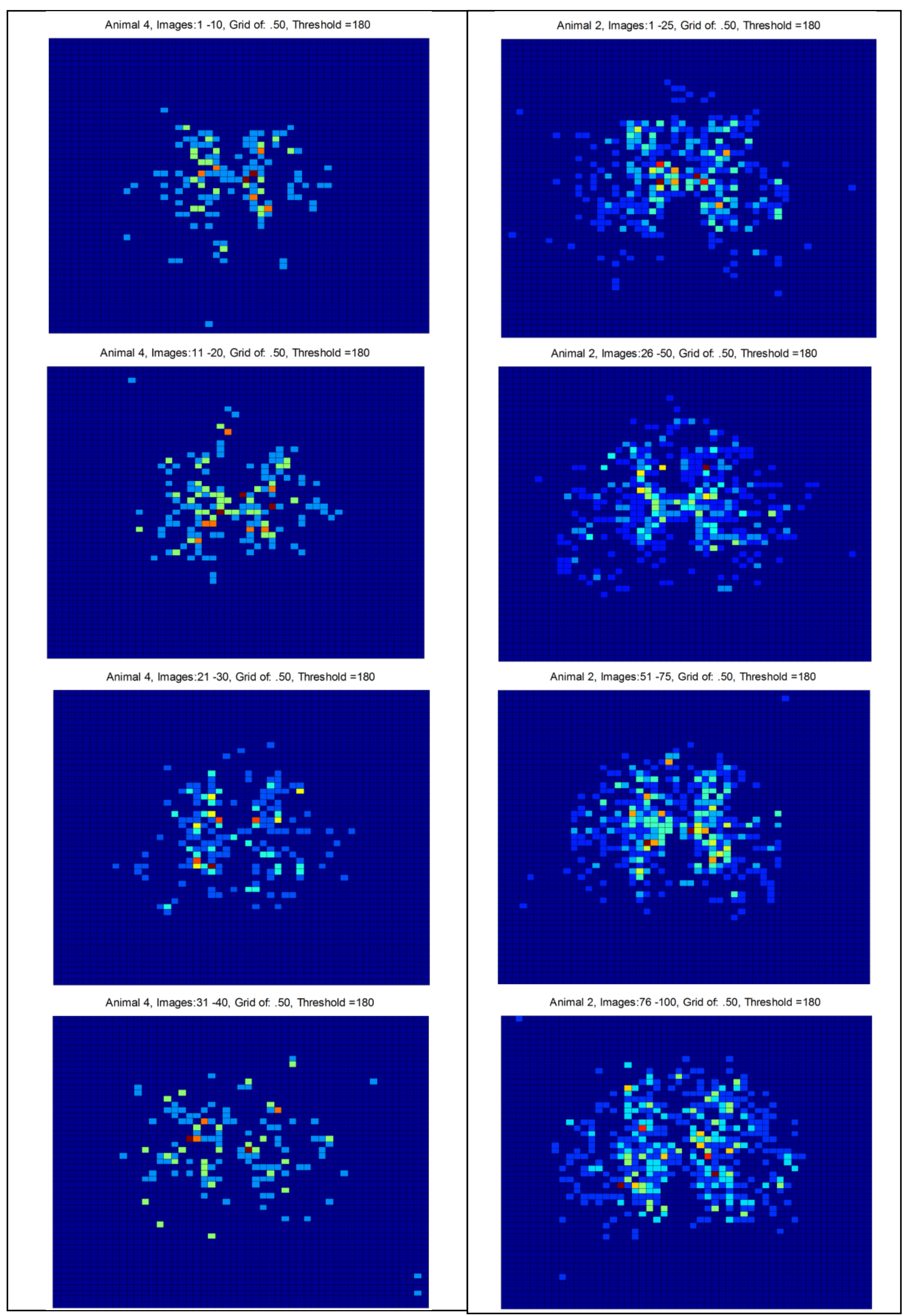




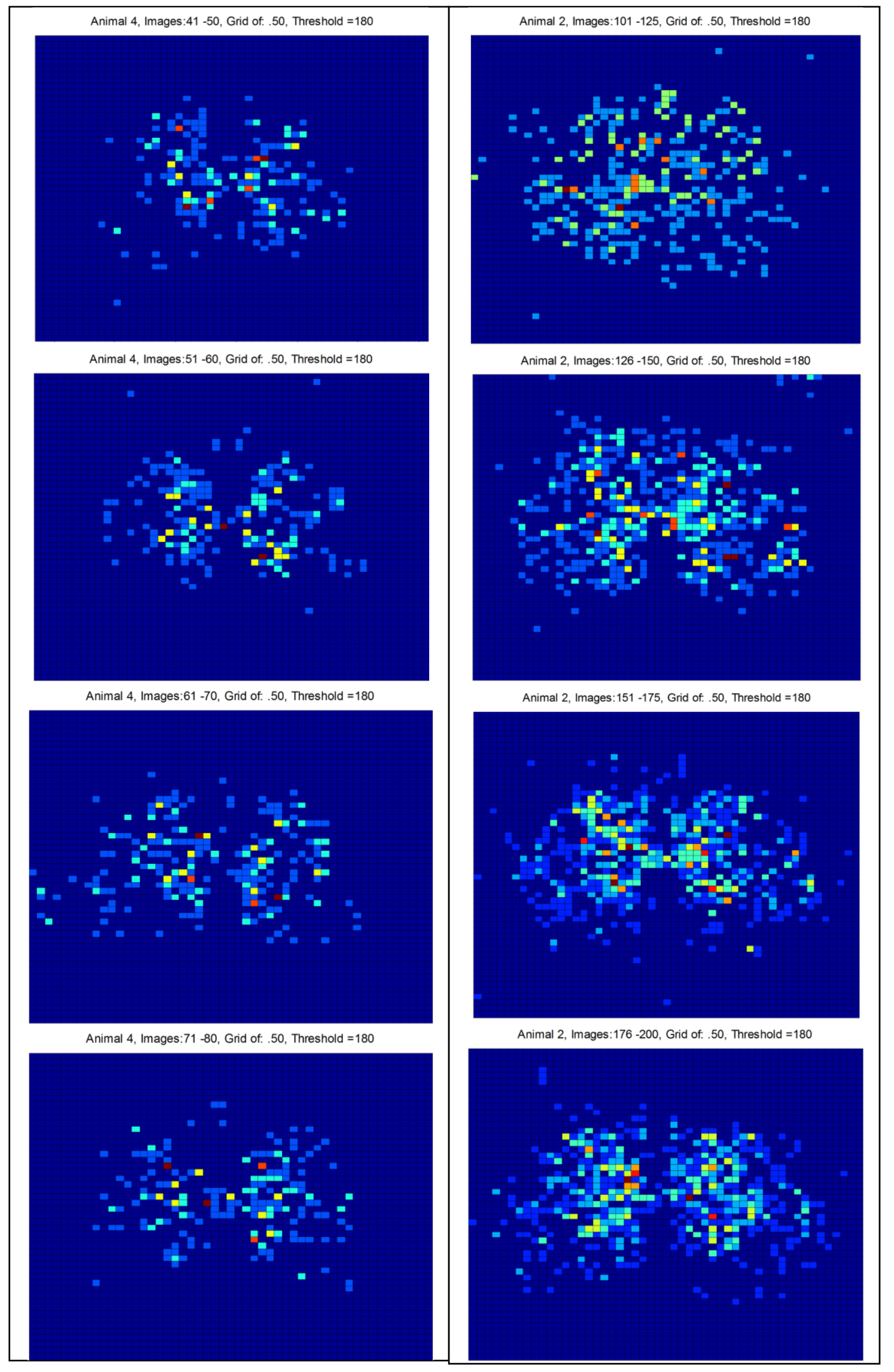




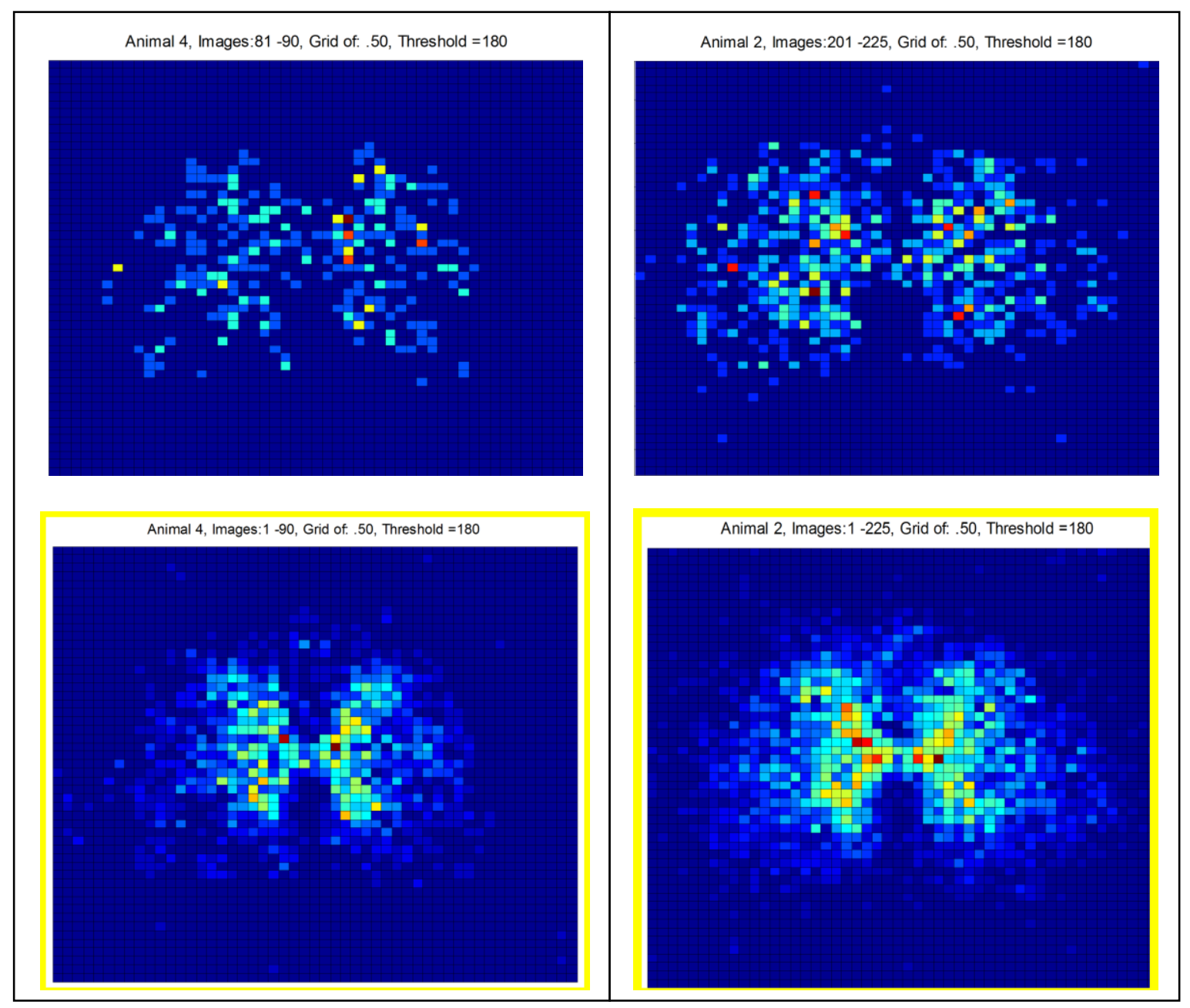


The final type of analysis was to illustrate quantification of centroid distribution density. This was done with MATLAB's hist3 function and colormaps are displayed beneath the histograms for clarity. These displays are beneficial because later statistical analysis will test significance of FM distribution. It will be helpful to visually identify the best areas to compare quickly. Figures 35 and 36 are examples of this type of display.

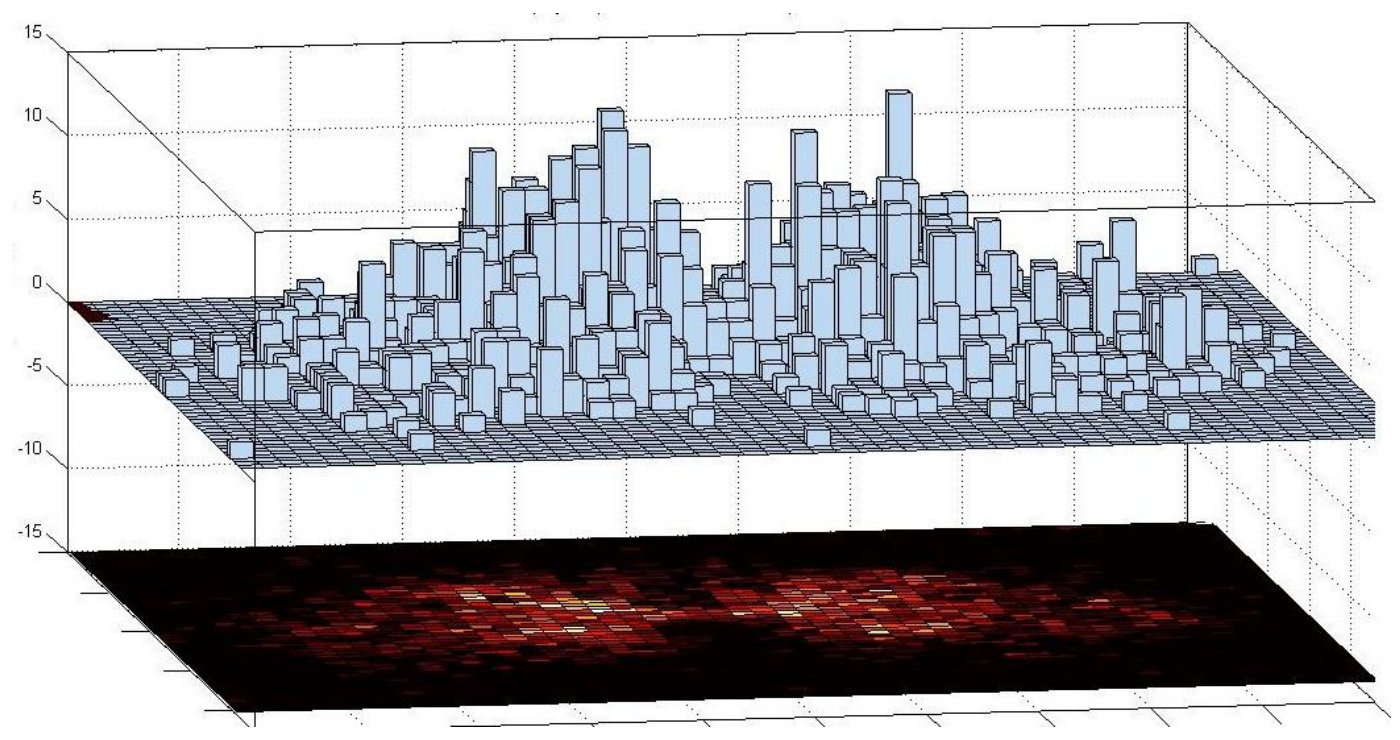

Figure 33: Colorplot of Animal 2. FM count is labeled on the z-axis. The $x$ - and y-axis correlate only to image orientation which is shown here with the anterior edge near and the posterior side in the background

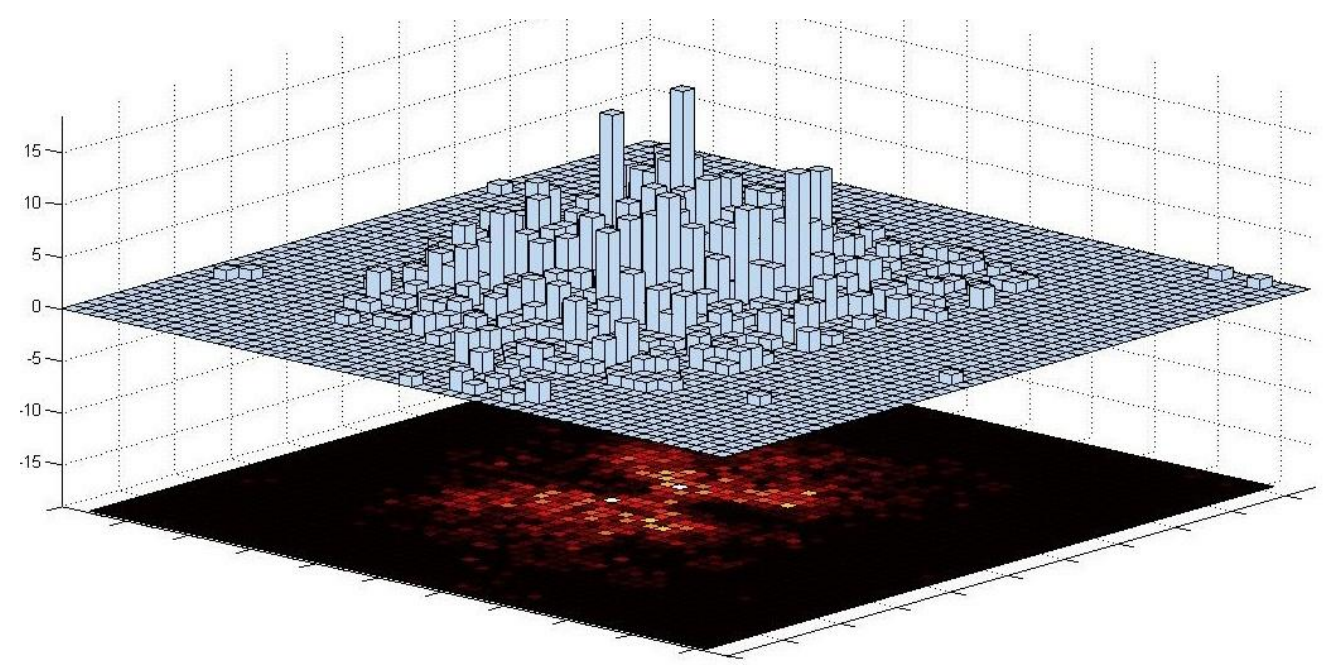

Figure 34: Colorplot of Animal 4. Axis are identical to Figure 35; the anterior direction is oriented in the foreground and slightly to the right. 
At this point, images from the injured animals were broken into five categories (see Table 4) based on proximity to the injury. The injury was done at T9 so it can be used as an anatomical reference point. Images the same distance away from the epicenter in different animals should correspond to the same spinal level. Color plots and 3D histograms of the combined data from each designated region are shown below. Figure 35 illustrates the region being analyzed relative to the whole spinal cord.

Table 4: Precise tissue designations defining the each region for color plot analysis; image file numbers are recorded on top, and below that is the calculated distance spanned by each segment in microns and italicized.

\begin{tabular}{|c|l|l|l|l|l|}
\hline $\begin{array}{c}\text { Animal } \\
\text { Number: }\end{array}$ & "Rostral" & $\begin{array}{l}\text { "Rostral } \\
\text { Penumbra" }\end{array}$ & "Epicenter" & $\begin{array}{l}\text { "Caudal } \\
\text { Penumbra" }\end{array}$ & "Caudal" \\
\hline 1 & $91-105$ & $106-111$ & $112-118$ & $119-124$ & $125-132$ \\
& 6550 & 2700 & 3150 & 2700 & 3600 \\
\hline 2 & $1-60$ & $61-89$ & $90-149$ & $150-169$ & $170-225$ \\
& 5490 & 2520 & 5400 & 1800 & 5040 \\
\hline 3 & $91-125$ & $126-140$ & $141-151$ & $152-171$ & \\
& 5625 & 3375 & 2475 & 4500 & \\
\hline
\end{tabular}

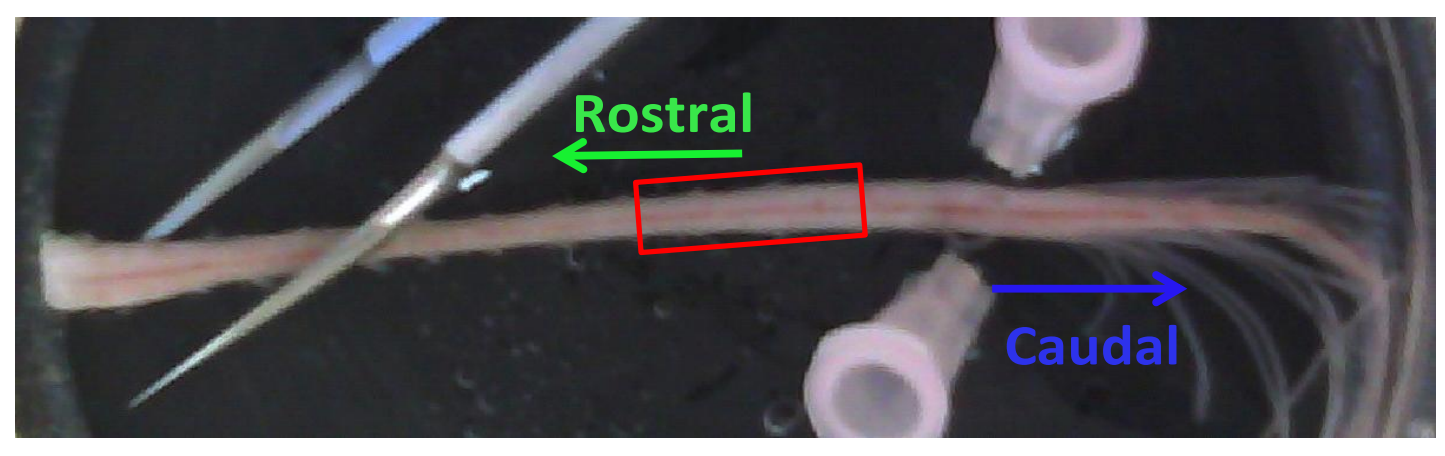

Figure 35: Uninjured spinal cord, before being cut into 4 even blocks. The third block is the tissue analyzed in this study. It contains the lower thoracic and upper lumbar segments. T9 is approximately at the center of the red block. 


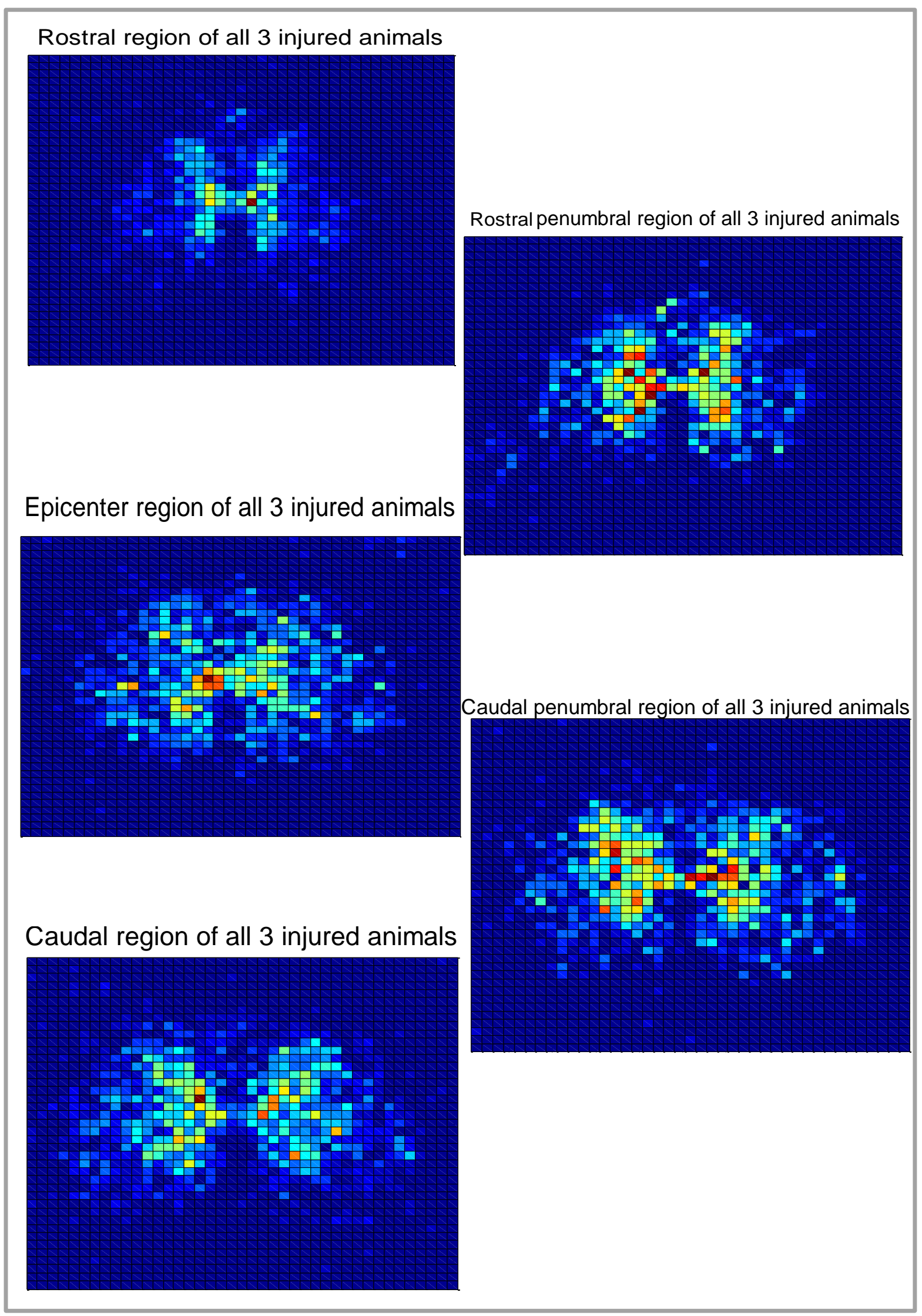

Figure 36: Colormap illustration of trends in FM distribution across injury epicenter. 


\section{Microsphere Solution Measurements}

Verification of the size and charge of one type of particle was done and the results compared to those provided by Spherotech. Size was measured by diluting an original sample of vortexed sphere solution diluted by a factor of 20 in a cuvette. The solution was measured using a dynamic light scattering (DLS) device (Malvern Zetasizer, Worcestershire, United Kingdom) and results are shown in Figure 37. The particles reported as $1.8 \mu \mathrm{m}$ in diameter were measured to most commonly occur as $1.533 \mu \mathrm{m}$ in diameter, with a range of 1.416 to $1.649 \mu \mathrm{m}$. The zeta potential was measured to be slightly negative, as expected, and is shown in Figure 38. This was done using the same sample, unchanged dilution, in a folded capillary cell from Malvern.

Available filters for the DLS had too small a pore size to run these analyses on the larger spheres, estimated by Spherotech to be $10.8 \mu \mathrm{m}$ in diameter. The difference between measured and reported sizes is small compared to the size of the blood vessels being measured and therefore the company's report is considered validated. It is not necessary to verify the size of the larger particles size using means such as an electron microscope since a comparable difference in these particles (an actual diameter being $.3 \mu \mathrm{m}$ different than the expected diameter) would have no measurable outcome on study results. 


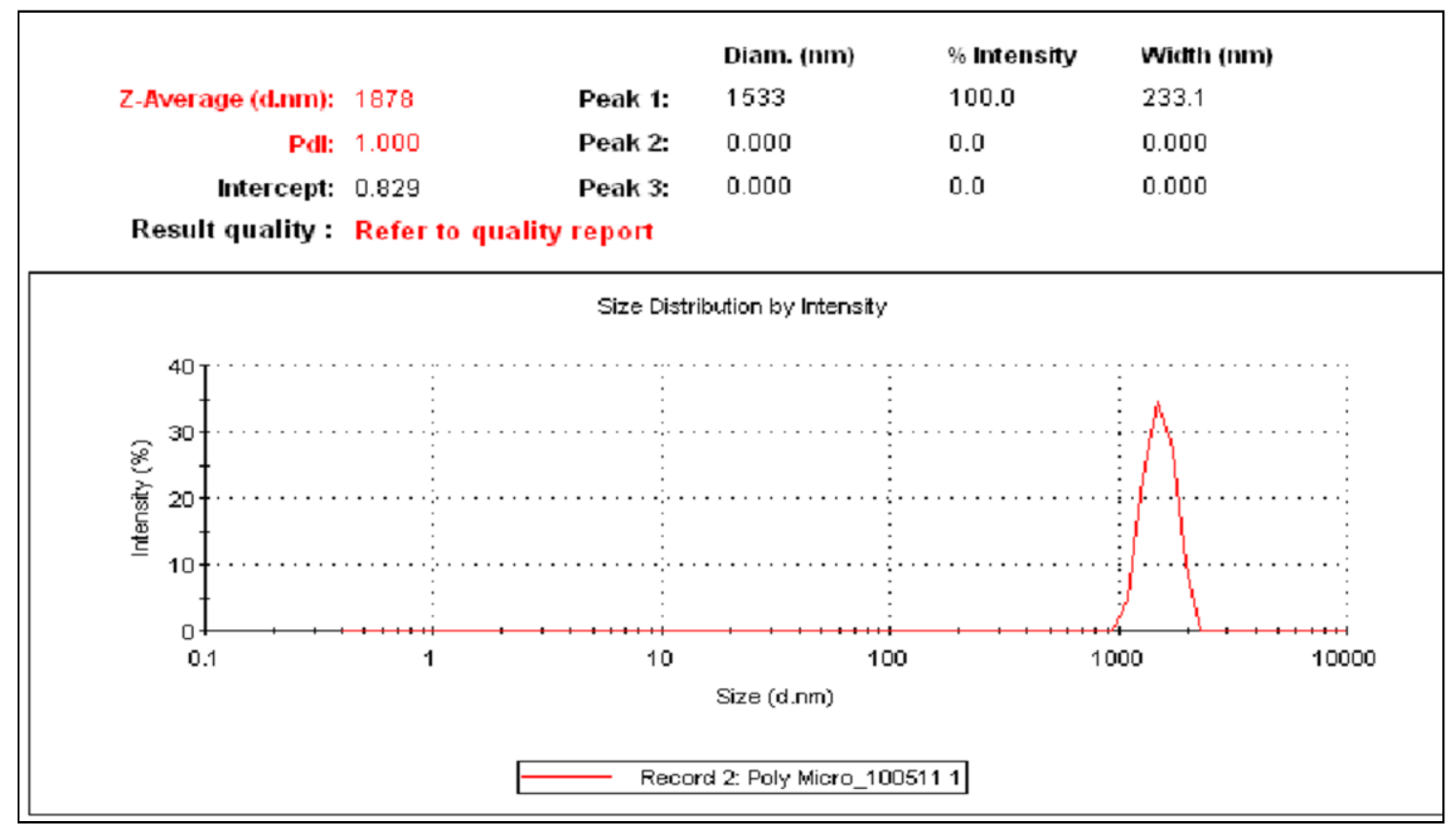

Figure 37: Particle size distribution for "1.8 micrometer diameter" particles.

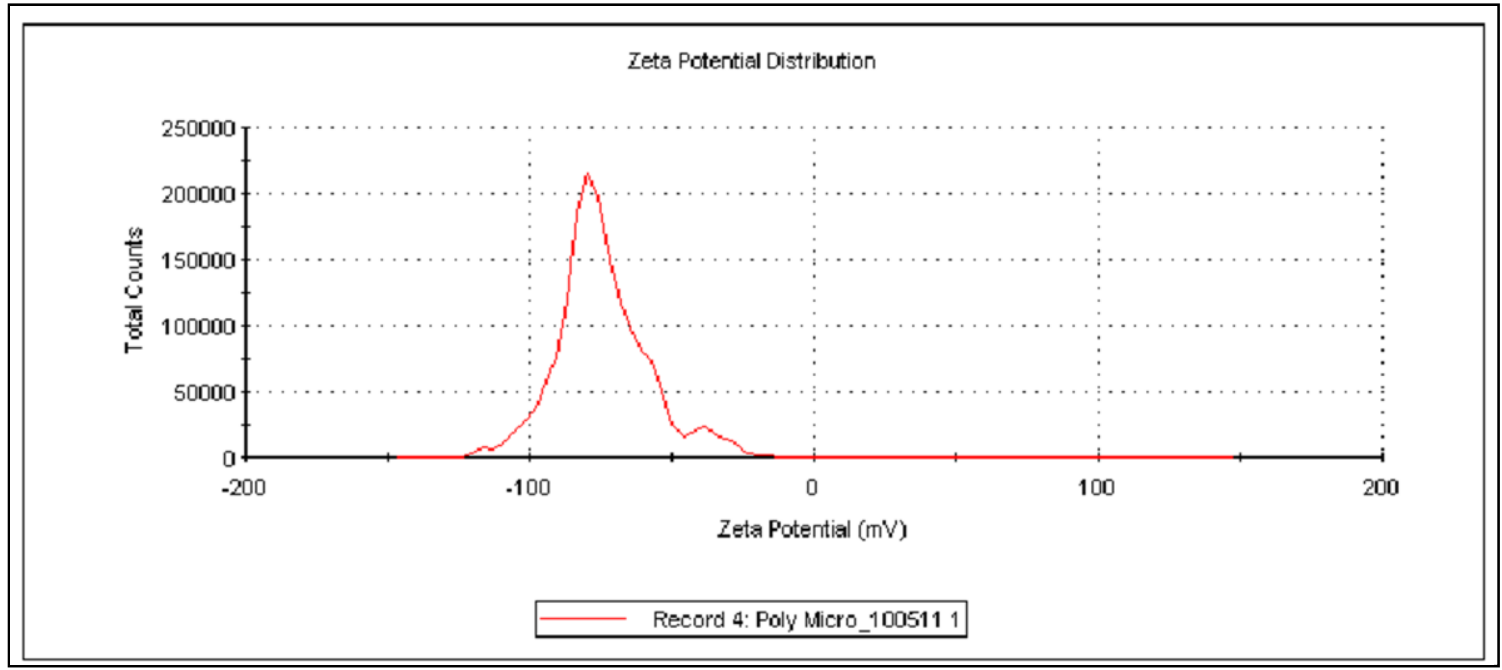

Figure 38: Zeta potential measurements for 1.8 micrometer particles. 


\section{CHAPTER 4: CONCLUSIONS}

\section{FM Injection Surgery, efficacy}

Addressing Specific Aim 1, injecting FMs into the left ventricle of an anesthetized rat is a highly reliable way to deliver the fluorescent particles to body tissue, including the spinal cord. Reliability is demonstrated by the fact that the number of particles per tissue sample is comparable between injured and, separately, between uninjured animals; animals within the same group are not statistically different from one another after undergoing the same FM injection procedure. Particle distribution in this study demonstrates that mixing in the blood is adequate for random delivery of FMs to tissue. Therefore, conclusions regarding relative blood flow, based on relative particle distribution, should be valid.

If a ventilator is not used to provide the animals with automated respiration, they will suffocate and the heart will stop its normal pumping rate and force within approximately 120 seconds of beginning FM injection surgery. This will result in an unreliable distribution of FMs and invalidate the above assumptions required for data analysis.

To cryosection tissue containing FMs it is necessary to set the section thickness at "several times the diameter of the particles" (De Visscher, Haseldonckx et al. 2006). However, sectioning at $90 \mu \mathrm{m}$ for the present studies led to complications. Even a dull cryostat blade will cut tissue at this thick setting so it was easy to continue using a blade well past the point at which it should have been replaced. When no longer sharp, the blade causes tissue sections to deform as they are cut. Deformed sections do not change the outcome of the total-sphere-count charts, but they reflect inaccurate patterns when analyzed with the spatial analysis program. Additionally, thick sections tend to float away from the glass slide when the slide is immersed in solution for rinsing, staining, or immunohistochemistry. To avoid these problems, it is suggested that 
specially coated slides be used for mounting, and more consideration be taken for the blade during sectioning.

Another option is to change protocols to adopt both a smaller sphere diameter and, consequently, a thinner cryostat section for further studies. Buckberg et al. describe spheres behaving most like red blood cells as they approached the actual diameter of the cells, at approximately $8 \mu \mathrm{m}$. Using $8 \mu \mathrm{m}$ particles would necessitate sections that are a minimum of 32 $\mu \mathrm{m}$ thick, which is almost identical to the $30 \mu \mathrm{m}$ setting most commonly used in the lab. An added benefit is that the tissue sections would not be likely to be lost during washes and all normal staining procedures could be done and easily compared to standard work.

\section{MATLAB Automated Analysis}

The automated counting program's FM identification is statistically equal to a human's counting. The statistical analysis showed that image background noise did affect the likelihood of miscounting. Exposure time and other digitally controlled parameters were kept constant but this was not enough. More reliable images would be captured if the qualities of the first image (prior to saving) were visually matched by the user with a pre-determined "ideal standard" image. Alternatively, the white-balance feature could be used and scaled to a brightly fluorescing sphere. This was not tested but should theoretically equalize the background fluorescence.

In future use of the program, code will be implemented that will automatically save new figures, using distinguishing user-defined parameters as the file name. This will make data analysis more efficient. 


\section{Longitudinal Analysis of FM Distribution}

The gross pattern in uninjured spinal cords is that of increasing FM detection approaching the caudal end of the region examined. This is an expected result since the tissue area is also larger here, moving closer to the lumbar enlargement of the cord (Somers 2001).

The single-image set from Animal 1, total image set from Animal 2, and both single and double sets from Animal 3 all show a decrease in the number of FMs detected at the injury epicenter, compared to surrounding tissue and compared to uninjured tissue at the same level. This is an expected result since, at day 7, the injury epicenter is still experiencing a high level of angiogenesis; the body would not be working hard to rebuild the vascular bed if tissue were already well perfused at this time point (Loy, Crawford et al. 2002; Dray, Rougon et al. 2009).

While the trend of decreasing blood supply near the injury was not unexpected, it was surprising that the result was significant in all three animals even though only one set of tissue was analyzed for Animal 1. This indicates that it is not necessary to image every section of tissue in order to get accurate information on the relative blood supply of the epicenter region. Future studies can use this type of protocol to get accurate FM data and still preserve tissue for histological stains or other analysis.

In looking specifically at Animal 3, the injury epicenter is not represented by the middle of the data set. This is because the cord was not blocked with the injury in the center of the block and does not indicate that the surgery or injury are actually varied compared to the others. The full epicenter area for Animal 3 is represented by the data here, shifted to the right on the $\mathrm{x}$-axis, and the next block of tissue in the caudal direction remains preserved for future analysis. When this tissue has been added there will be stronger information regarding blood supply in the upper lumbar region after spinal cord injury. The data from the first two animals suggests that it is elevated but this can only be analyzed for a few millimeters of tissue at this point.

It was not expected that any tissue would have more FMs compared to the controls. All three of the injured spinal cords did have more particles, even hundreds of microns from the 
injury. There is a possibility that this is due to some procedural difference. The FM surgery groups were run on different days. But the protocols were identical (anesthesia, ventilation, surgery procedures, FM solution preparation and administration, and dissection). To make sure this is not the source of the difference, future studies should be planned so that surgery times are randomized. Incorporating FMs into a temporal study would help explain some of the physiologic mechanism behind the apparent increase in blood supply to the spinal cord very far from the injury site.

\section{Cross Section Analysis is FM Distribution}

An extensive literature search reflects that blood is supplied to the spinal cord by one of two primary routes. The ventral spinal artery supplies the ventral 2/3 (by area) and pair of posterior spinal arteries supply the posterior 1/3 (see Figure $1 \&$ Figure 39). Vasculature is discussed in terms of its anatomical structure, but not its actual functionality. Even though it is widely understood that grey matter has a higher metabolic rate than white matter, they are often described as being equal vascular. The color plots calculated here reflect that in healthy tissue, very few FMs embolize vessels in the white matter and this trend does not appear to reflect a preference for vessels having originated in either the anterior or posterior (or other) arteries.

Figure 39 illustrates accepted anatomy of the spinal cord blood supply overlapping actual relative blood supply as measured by this work. The distinctions typically used to describe SC blood vessel anatomy do not seem to apply to actual blood supply. Whether the FMs were delivered from anterior or posterior arteries cannot be determined from their distribution. The actual distribution in healthy tissue seems entirely reflective of grey matter. 


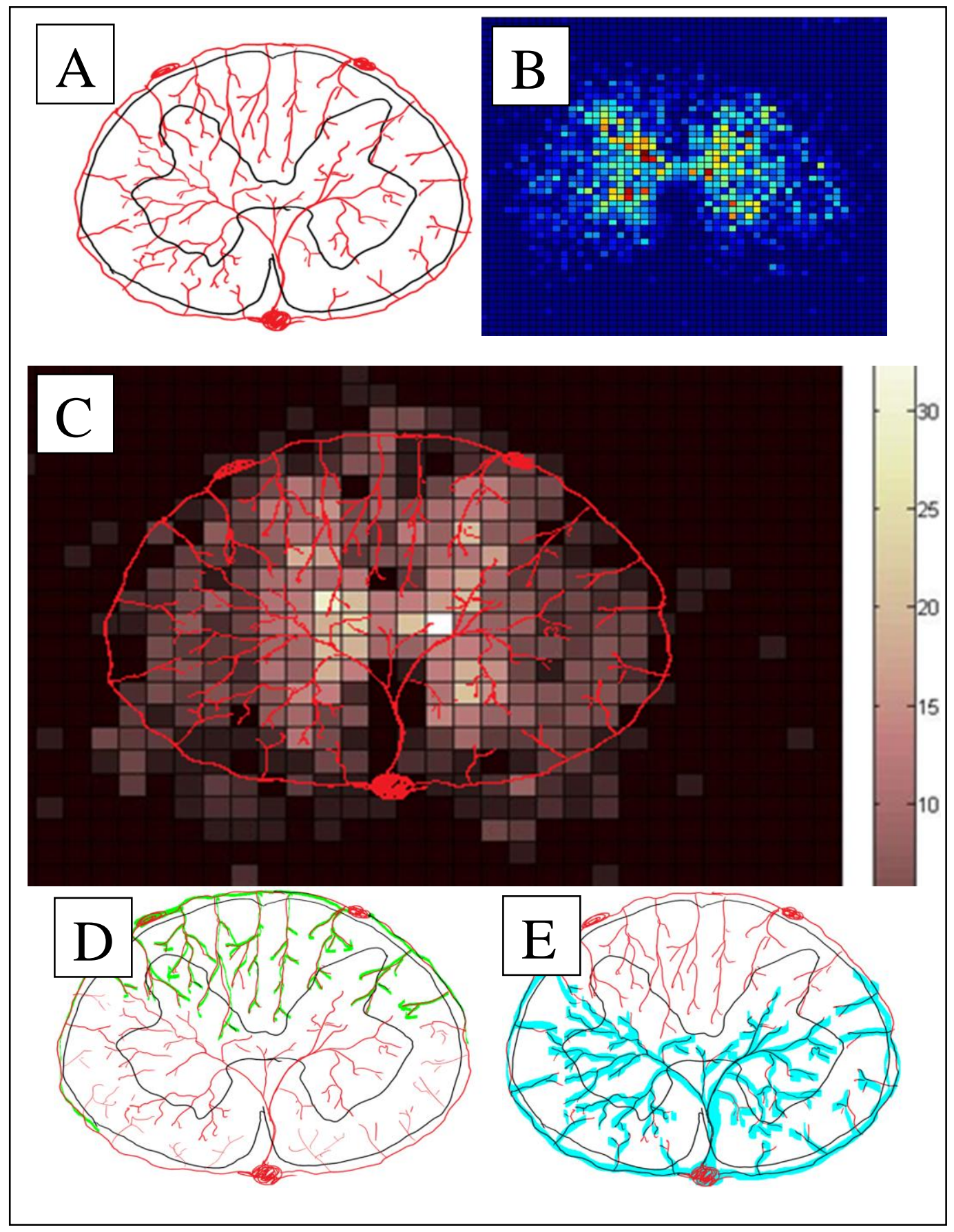

Figure 39: Vasculature versus Blood Flow - (A) structure, adapted from Anesthesia UK (B) blood flow as measured in this study (C) blood flow re-colored with vascular pattern overlay, and approximate region supplied with blood by the posterior arteries (D) and anterior artery (E). 


\section{Future Directions}

A study investigating the temporal relationship of blood supply after spinal cord injury is planned. This could be designed to match other studies in the center with regard to exact time points and injury model.

Further studies may use two colors of FMs which are equal in size but administered sequentially so it can be determined whether the vessels embolized are oriented from the inside out or from the outside in. This would help explain the pattern of blood flow in healthy tissue, since that is not apparent from the data here, and would lead to more interesting comparisons in the injured tissue.

Future MATLAB programming should be written to be more robust. Code could be rewritten so that if an image is blank (no spheres identified) or is missing entirely, the code will create a data point which is the average of the previous and subsequent images (for the bar graph outputs). For spatial analysis, it should automatically replace missing images with previous image. It should display a list of images that were replaced for the user to see. This type of work will make it easier for other people to use the same analysis program later on.

The tissue analyzed here is actually marked with LEA. Soon, the LEA patterns will be analyzed and compared to FM distribution. LEA is not normally analyzed in such thick tissue sections but the thickness actually shows vessels shapes and details that are destroyed with thinner sectioning. This thick tissue should make calculations of vessel geometry more accurate. 


\section{REFERENCES}

(2004). "Spinal cord." 2011, from www.frca.co.uk/article.aspx?articleid=100360.

AJH, R. Tween (R) 20. Product Number P1379, Sigma-Aldrich, Saint Louis, Missouri.

Alm, A. and A. Bill (1973). "Ocular and optic nerve blood flow at normal and increased intraocular pressures in monkeys (Macaca irus): a study with radioactively labelled microspheres including flow determinations in brain and some other tissues." Experimental Eye Research 15(1): 15-29.

Awad, H., D. P. Ankeny, et al. (2010). "A Mouse Model of Ischemic Spinal Cord Injury with Delayed Paralysis Caused by Aortic Cross-clamping." Anesthesiology 113(4): 880-891 810.1097/ALN.1090b1013e3181ec1061ee.

Benton, R. L., M. A. Maddie, et al. (2008). "Transcriptomic screening of microvascular endothelial cells implicates novel molecular regulators of vascular dysfunction after spinal cord injury." J Cereb Blood Flow Metab 28(11): 1771-1785.

Biglioli, P., M. Roberto, et al. (2004). "Upper and lower spinal cord blood supply: the continuity of the anterior spinal artery and the relevance of the lumbar arteries." The Journal of Thoracic and Cardiovascular Surgery 127(4): 1188-1192.

Buckberg, G. D., J. C. Luck, et al. (1971). "Some sources of error in measuring regional blood flow with radioactive microspheres." Journal of Applied Physiology 31(4): 598-604.

Carpenter, A., T. Jones, et al. (2006). "CellProfiler: image analysis software for identifying and quantifying cell phenotypes." Genome Biology 7(10): R100.

Cerutti, C., M. P. Gustin, et al. (2001). "Beat-to-beat stroke volume estimation from aortic pressure waveform in conscious rats: comparison of models." American Journal of Physiology - Heart and Circulatory Physiology 281(3): H1148-H1155.

Cicutti, N. and K. Rakusan (1992). "Microvascular flow vectors in normal and hypertrophic myocardium as determined by the method of colored microspheres." Microvascular Research 43(3): 267-275.

De Visscher, G., M. Haseldonckx, et al. (2006). "Fluorescent microsphere technique to measure cerebral blood flow in the rat." Nat. Protocols 1(4): 2162-2170.

Domenech, R. J., J. I. Hoffman, et al. (1969). "Total and Regional Coronary Blood Flow Measured by Radioactive Microspheres in Conscious and Anesthetized Dogs." Circulation Research 25(5): 581-596.

Dommisse, G. F. (1974). "THE BLOOD SUPPLY OF THE SPINAL CORD: A Critical Vascular Zone in Spinal Surgery." J Bone Joint Surg Br 56-B(2): 225-235.

Dray, C., G. Rougon, et al. (2009). "Quantitative analysis by in vivo imaging of the dynamics of vascular and axonal networks in injured mouse spinal cord." Proceedings of the National Academy of Sciences 106(23): 9459-9464.

encyclopediascience. (2011). "Nervous System." 2011, from http://encyclopediascience.wordpress.com/2011/05/02/nervous-system/.

Fawcett, J. W. and R. A. Asher (1999). "The glial scar and central nervous system repair." Brain Research Bulletin 49(6): 377-391.

Hebel, R. and M. W. Stromberg (1986). Anatomy and Embryology of the Laboratory Rat. Worthsee, Federal Republic of Germany, M.W. BioMed Verlag.

Lee, H. B. and M. D. Blaufox (1985). "Blood Volume in the Rat." Journal of Nuclear Medicine 26(1): $72-76$. 
Loy, D. N., C. H. Crawford, et al. (2002). "Temporal progression of angiogenesis and basal lamina deposition after contusive spinal cord injury in the adult rat." The Journal of Comparative Neurology 445(4): 308-324.

Magnuson, D. S. K., R. Lovett, et al. (2005). "Functional Consequences of Lumbar Spinal Cord Contusion Injuries in the Adult Rat." Journal of Neurotrauma 22(5): 529-543.

Marcus, M., D. Heistad, et al. (1977). "Regulation of total and regional spinal cord blood flow." Circulation Research 41(1): 128-134.

McDonald, J. W. and C. Sadowsky (2002). "Spinal-cord injury." The Lancet 359(9304): 417-425.

Morita, Y., B. D. Payne, et al. (1990). "Local blood flow measured by fluorescence excitation of nonradioactive microspheres." American Journal of Physiology - Heart and Circulatory Physiology 258(5): H1573-H1584.

Purves, D., G. J. Augustine, et al., Eds. (2001). Neuroscience. Sunderland (MA), Sinauer Associates.

Scheff, S. W., A. G. Rabchevsky, et al. (2003). "Experimental Modeling of Spinal Cord Injury: Characterization of a Force-Defined Injury Device." Journal of Neurotrauma 20(2): 179193.

Somers, M. F. (2001). Spinal Cord Injury Functional Rehabilitation, Appleton \& Lange.

Thomas K, H. (1988). "The physiology of wound healing." Annals of Emergency Medicine 17(12): 1265-1273.

Tonnesen, M. G., X. Feng, et al. (2000). "Angiogenesis in Wound Healing." J Investig Dermatol Symp Proc 5(1): 40-46.

Tycko, D. H., M. H. Metz, et al. (1985). "Flow-cytometric light scattering measurement of red blood cell volume and hemoglobin concentration." Appl. Opt. 24(9): 1355-1365.

Urdzíková, L., P. Jendelová, et al. (2006). "Transplantation of Bone Marrow Stem Cells as well as Mobilization by Granulocyte-Colony Stimulating Factor Promotes Recovery after Spinal Cord Injury in Rats." Journal of Neurotrauma 23(9): 1379-1391.

Yamakami, I. and T. K. McIntosh (1989). "Effects of Traumatic Brain Injury on Regional Cerebral Blood Flow in Rats as Measured withRadiolabeled Microspheres." J Cereb Blood Flow Metab 9(1): 117-124.

Zhang, Y. P., D. A. Burke, et al. (2008). "Spinal Cord Contusion Based on Precise Vertebral Stabilization and Tissue Displacement Measured by Combined Assessment to Discriminate Small Functional Differences." Journal of Neurotrauma 25(10): 1227-1240. 


\section{CURRICULUM VITAE}

RUTHIE S. FLIGOR

1823 S. Third St., Apt 3

Louisville, KY 40208

ruthie.fligor@gmail.com

Education:

M. Eng. in Bioengineering

Expected December 2011

\section{B.S. in Bioengineering}

J.B. Speed School of Engineering, UofL, Louisville KY

May 2011

\section{High School Diploma}

Model Laboratory High School, Richmond Kentucky

May 2005

Awards or Honors:

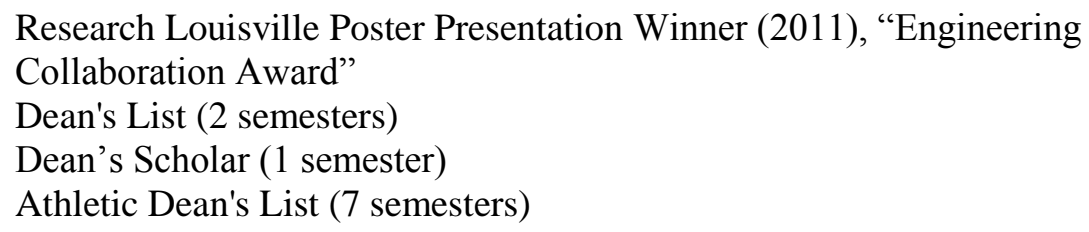

Employment History:

\section{Kentucky Spinal Cord Injury Research Center Louisville, KY \\ BioEnineering Co-op Student (3 non-consecutive semesters)}

- Produced preliminary designs for a novel ground-reaction-force detection system

- Collaborated with other engineering students and engineering faculty to finalize design and order custom system

- Implemented basic LabVIEW code for reading, displaying, saving, and automatically analyzing system data

- Designed follow-up study to compare spinal transcection to severe injury model; carried out study

- Performed essential lab work including animal care, animal surgeries, and preparing data for publication

- Participated in trainee events such as grand rounds seminars and weekly journal club meetings 
Lectures:

KSCIRCWeekly Seminar

Kinetic Assessment Tool Development, and Master's Thesis Work Presented to Presented to researchers, research staff, trainees \& clinical team members of KSCIRC, Frazier Rehab, and UofL Department of Neurosurgery

August 4, 2011

Introduction to the work of Jose Adams

Broad introduction to the body of work Dr. Adams has pioneered in pediatric cardiology; critical evaluation and discussion of one paper; prepared contextual background for Dr. Adams visit to the class Presented to graduate level class, Cardiovascular Dynamics I June 6, 2011

Journal Club lecture Hypoxia-inducible factor-1 (HIF-1)-independent microvascular angiogenesis in the aged rat brain (LaManna); provided background and critical evaluation/ discussion of the present work.

Presented toP.I.'s and trainees from KSCIRC and Frazier Rehab February 22, 2010

\section{KSCIRC/BE Symposium}

Development of an Accelerometer-based Kinetic Measurement System Presented to researchers, research staff, trainees \& clinical team members of KSCIRC, Frazier Rehab, and UofL Department of Neurosurgery

August 8, 2009

\section{Journal Club lecture}

Facilitation of Stepping with Epidural Stimulation in Spinal Rats: Role of Sensory Input (Igor Lavrov, Gre'goireCourtine, Christine J. Dy, Rubia van den Brand, Andy J. Fong, Yuri Gerasimenko, HuiZhong, Roland R. Roy, and V. Reggie Edgerton)

Presented toP.I.'s and trainees from KSCIRC and Frazier Rehab December 9, 2008

Conferences Attended:

KSCHIRT Symposium - 2009 \& 2011

Research Louisville - 2009-2011

Neuroscience Day, Louisville - 2009 \& 2010

Idea Festival - 2011

Nanotechnology and Nanomedicine Symposium - 2009

Extracurricular Activities\& Societies:

UofL Rowing (Novice 1 year, Varsity 3 years)

BMES Member (served as secretary for 1 year) 
Engineering World Health

Invited to join Golden Key and Tau Beta Pi honor societies

Community Service:

UofL Hospital Volunteer (January 2011 - present)

Project Warm (2007-2010, Louisville, KY)

Festival of Trees and Lights (2006-2010, Louisville, KY) 
APPENDIX I

I
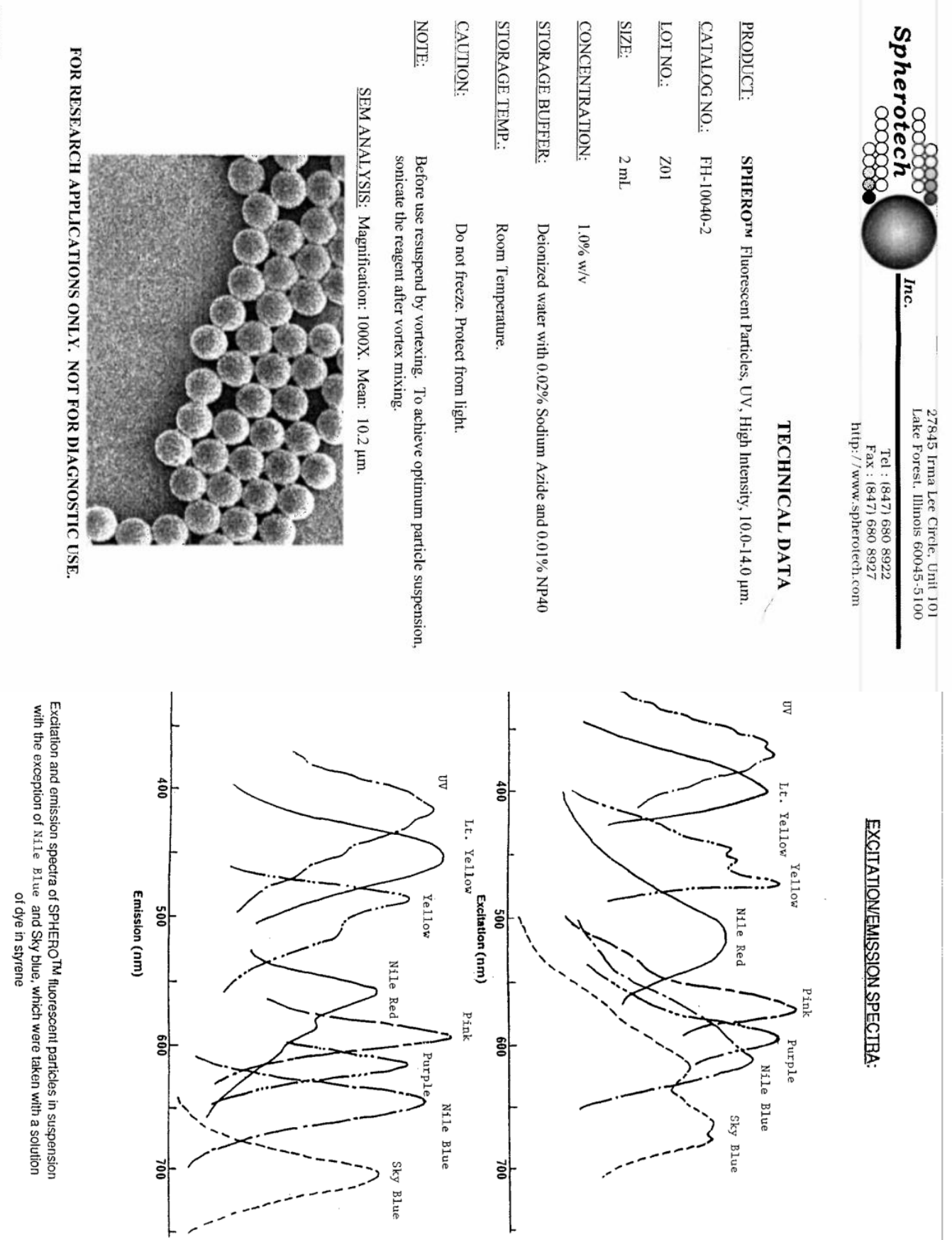

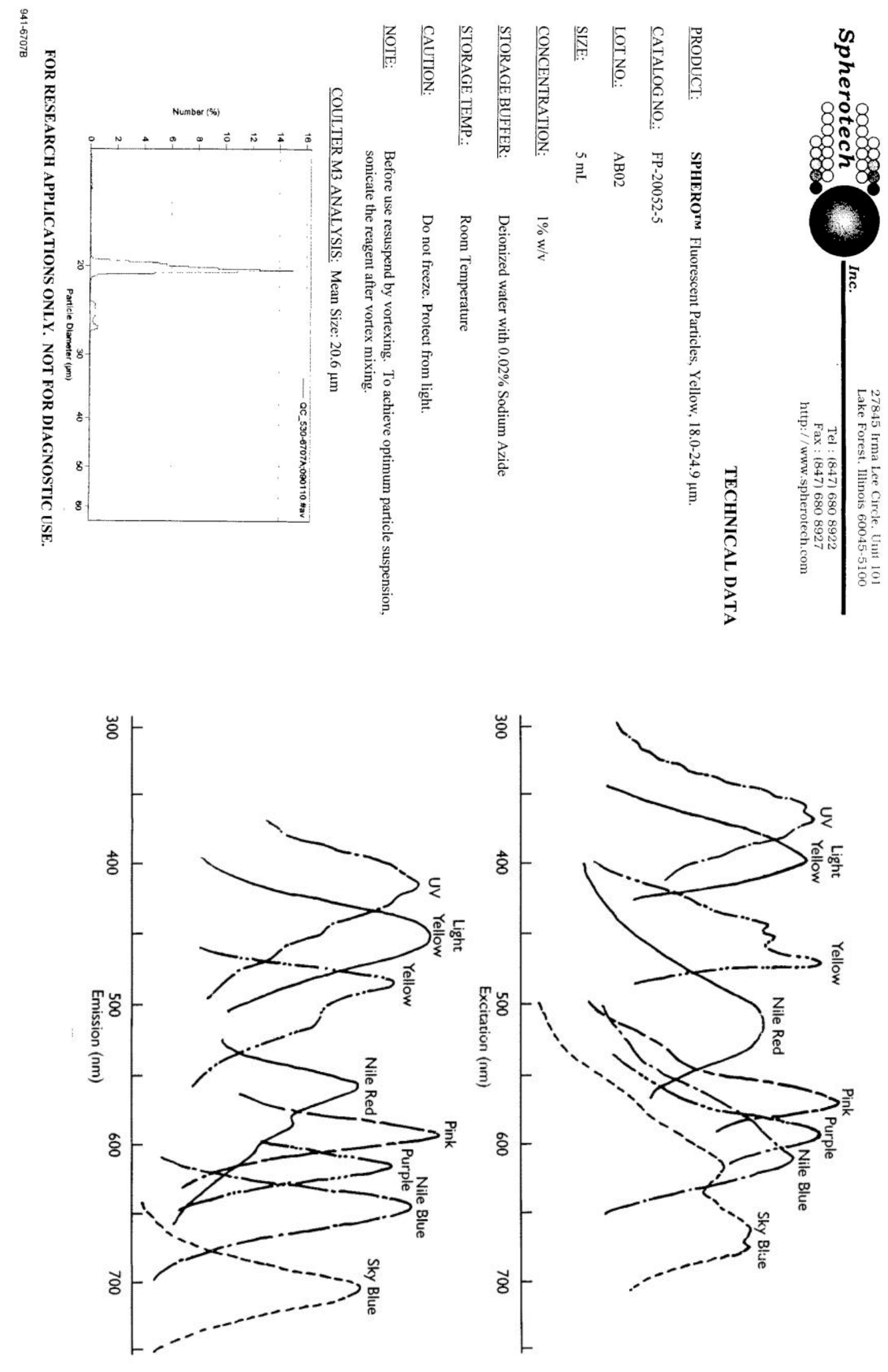
APPENDIX II

Table 5: Animal use summary

Ventricular Injections

\begin{tabular}{llllllr}
\hline Animal \# & $\begin{array}{l}\text { (Animal \# } \\
\text { in study) }\end{array}$ & Date & Spheres used & LEA & Notes & Status of data \\
1 & & $9 / 23 / 10$ & $10 \mu \mathrm{m}, 20 \mu \mathrm{m}$ & No & & Sectioned (red slide box) \\
2 & & $10 / 22 / 10$ & $2 \mu \mathrm{m}, 10 \mu \mathrm{m}, 20 \mu \mathrm{m}$ & No & Sectioned partially \\
3 & & $11 / 23 / 10$ & $20 \mu \mathrm{m}$ & Yes & & All sectioned (red slide box) \\
4 & & $11 / 23 / 10$ & $2 \mu \mathrm{m}$ & No & & Sectioned P1, P2 (red slide box) \\
5 & "1" & $5 / 2 / 11$ & $2 \mu \mathrm{m}, 10 \mu \mathrm{m}$ & Yes & Injured (IH 150) & All of cord sectioned; blue imaged \& analyzed \\
6 & "2" & $5 / 2 / 11$ & $2 \mu \mathrm{m}, 10 \mu \mathrm{m}$ & Yes & Injured (IH 150) & Blocked \\
7 & "3" & $5 / 2 / 11$ & $2 \mu \mathrm{m}, 10 \mu \mathrm{m}$ & Yes & Injured (IH 150) & In sucrose \\
8 & "4" & $5 / 2 / 11$ & $2 \mu \mathrm{m}, 10 \mu \mathrm{m}$ & Yes & Injured (IH 150) & All of cord sectioned; partially imaged \\
9 & "5" & $5 / 2 / 11$ & $2 \mu \mathrm{m}, 10 \mu \mathrm{m}$ & Yes & Uninjured & All of cord sectioned; partially imaged \\
10 & "6" & $5 / 2 / 11$ & $2 \mu \mathrm{m}, 10 \mu \mathrm{m}$ & Yes & Uninjured & \\
\hline
\end{tabular}

\section{Aortic Injections}

\begin{tabular}{|c|c|c|c|c|c|}
\hline Animal \# & & Date & Spheres used & Notes & \\
\hline Practice 1 & & $6 / 8 / 11$ & $\mathrm{n} / \mathrm{a}$ & & Petruska animal; bled out, no injection \\
\hline Practice 2 & & $6 / 27 / 11$ & $20 \mu \mathrm{m}$ & & Petruska's; blocked, sectioned, very very few in cord \\
\hline 3 & "1" & $8 / 19 / 11$ & $2 \mu \mathrm{m}, 10 \mu \mathrm{m}$ & & Blocked, sectioned lumbar transverse; bad surgery \\
\hline 4 & "2" & $8 / 19 / 11$ & $2 \mu \mathrm{m}, 10 \mu \mathrm{m}$ & & Blocked, sectioned lumbar transverse; bad surgery \\
\hline 5 & & 9/7/11 & $2 \mu \mathrm{m}, 10 \mu \mathrm{m}$ & & Blocked, sectioned lumbar sagittally, not successful \\
\hline
\end{tabular}

\begin{tabular}{|c|c|c|c|c|}
\hline \multicolumn{5}{|c|}{ Ventilated Ventricular Injections } \\
\hline Animal \# & Date & Spheres & LEA & \\
\hline 1 & 10/7/11 & $2 \mu \mathrm{m}, 10 \mu \mathrm{m}$ & Yes & Uninjured \\
\hline 2 & 10/7/11 & $2 \mu \mathrm{m}, 10 \mu \mathrm{m}$ & Yes & Uninjured \\
\hline 3 & $10 / 7 / 11$ & $2 \mu \mathrm{m}, 10 \mu \mathrm{m}$ & Yes & Uninjured \\
\hline 4 & $10 / 12 / 11$ & $2 \mu \mathrm{m}, 10 \mu \mathrm{m}$ & Yes & Injured (IH 150); spheres 7 days later \\
\hline 5 & $10 / 12 / 11$ & $2 \mu \mathrm{m}, 10 \mu \mathrm{m}$ & Yes & Injured (IH 150); spheres 7 days later \\
\hline 6 & $10 / 12 / 11$ & $2 \mu \mathrm{m}, 10 \mu \mathrm{m}$ & Yes & Injured (IH 150); spheres 7 days later \\
\hline
\end{tabular}




\section{APPENDIX III}

\section{Cardiac Cycle Analysis}

Mean SV in a rat is shown to be $200 \mu \mathrm{L}$. Work in our lab, using a custom-build heart rate monitor for rats, shows that the resting heart rate of a rat is approximately 350 beats per minute.

Therefore:

$$
(200 \mu L)\left(350 \frac{\text { beats }}{\text { minute }}\right)=70,000 \frac{\mu L}{\min }=70 \frac{\mathrm{mL}}{\min }
$$

Lee and Blaufox (1985) measure blood volume for a rat and show that it varies predictably according to weight. It is reasonable to approximate the blood volume as $13 \mathrm{~mL}$ for the rats used in these studies (typical weight at time of experiment $=220 \mathrm{~g}$ ).

$$
\frac{13 \mathrm{~mL} \text { blood, total }}{70 \frac{\mathrm{mL}}{\mathrm{min}}}=.1786 \mathrm{~min}=10.7 \text { seconds }
$$

\section{Relative and Absolute Sphere Concentration}

Based on the technical characteristics provided by Spherotech, the number of microspheres per volume of solution is calculated as 18.189 (diameter) ${ }^{-3}$ where diameter is given in $\mu \mathrm{m}$. Therefore, for $1.81 \mu \mathrm{m}$ particles, the total number of spheres per $\mathrm{mL}$ is $3.07 \times 10^{9}$. There are $1.71 \times 10^{7}$ particles per $\mathrm{mL}$ in the $10.2 \mu \mathrm{m}$ particle solution. Calculations represent a total injection of $3 \times 10^{6}$ microspheres $(10.2 \mu \mathrm{m}$ blue fluorescing spheres) and $22.5 \times 10^{6}$ microspheres (1.8 $\mu \mathrm{m}$ red fluorescing spheres). For $220 \mathrm{~g}$ rat, blood volume $=13 \mathrm{~mL}$ (Lee and Blaufox 1985). Tycko et al. (1985) show that the volume of one red blood cell (RBC) is $90 f \mathrm{~L}$ (Tycko, Metz et al. 1985) and so, using the $42 \%$ hematocrit measured by Lee $\&$ Blaufox, it is true that:

$$
(.42, \text { Hematocrit }) x(13 \mathrm{~mL} \text { blood, total })=5.2 \mathrm{~mL}, \mathrm{RBC} \text { total volume }
$$


$10.2 \mu \mathrm{m}$ spheres (BLUE):

$$
\frac{3 \times 10^{6} \text { spheres }}{13 \mathrm{~mL} \text { blood }}=2.31 \times 10^{5} \frac{\text { spheres }}{\mathrm{mL}}
$$

$1.8 \mu \mathrm{m}$ spheres (RED):

$$
\frac{22.5 \times 10^{6} \text { spheres }}{13 \mathrm{~mL} \text { blood }}=1.73 \times 10^{6} \frac{\text { spheres }}{\mathrm{mL}}
$$

$\underline{\text { Red blood cells (RBC's): }}$

$$
\begin{aligned}
\frac{5.2 \times 10^{-3} L(R B C \text { Volume })}{90 \times 10^{-15} L(1 R B C)} & =5.57 \times 10^{10} \text { total RBC in rat } \\
\frac{5.78 \times 10^{10} \text { cells }}{13 \mathrm{~mL} \text { blood }} & =4.45 \times 10^{9} \frac{\mathrm{cells}}{\mathrm{mL}}
\end{aligned}
$$

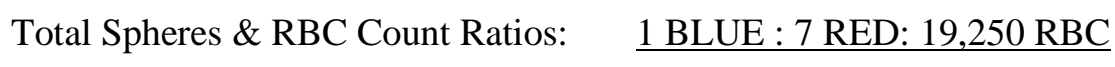

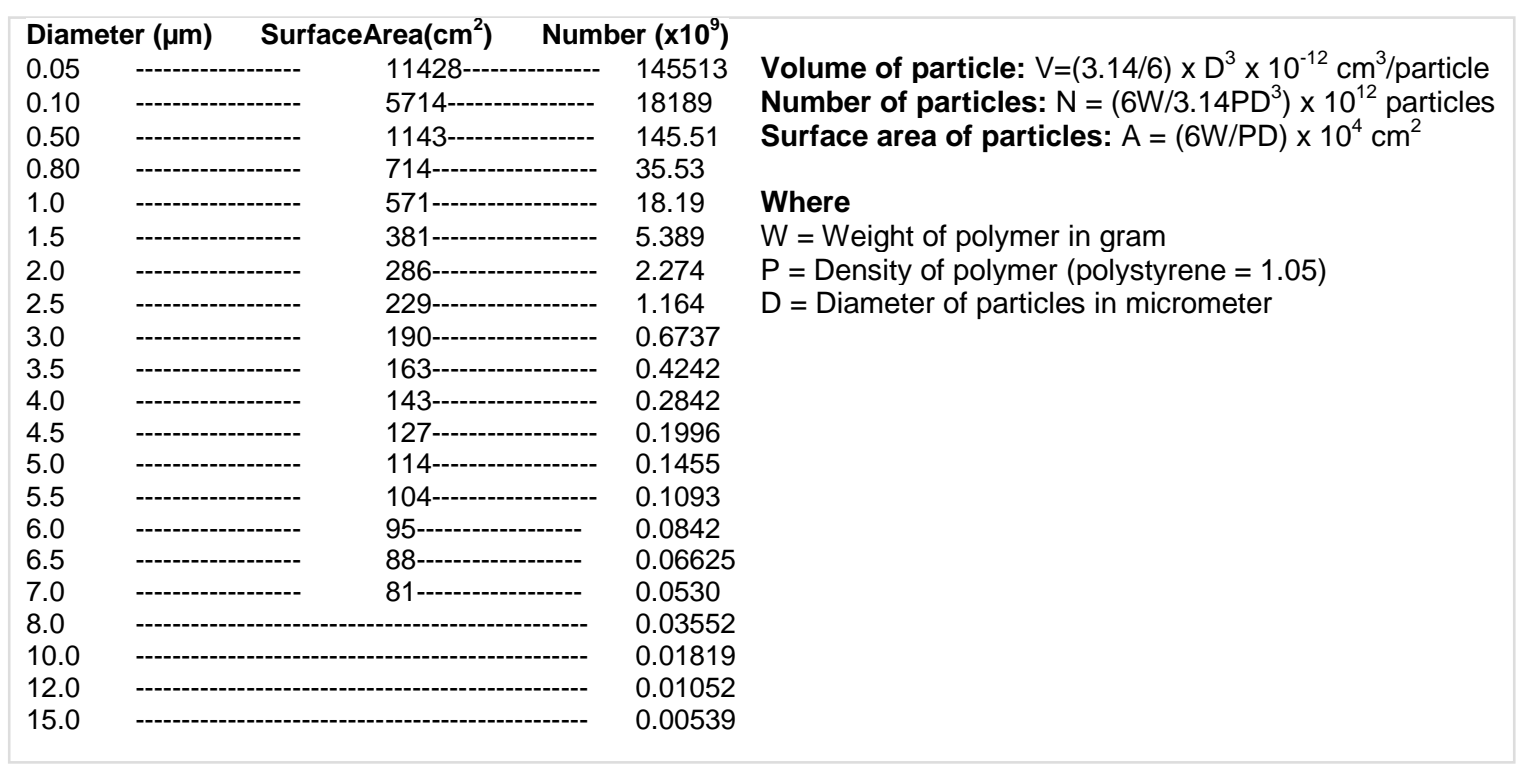

Figure 40: Surface area, absolute count, and particle volume formulas provided by Spherotech 


\section{APPENDIX IV}

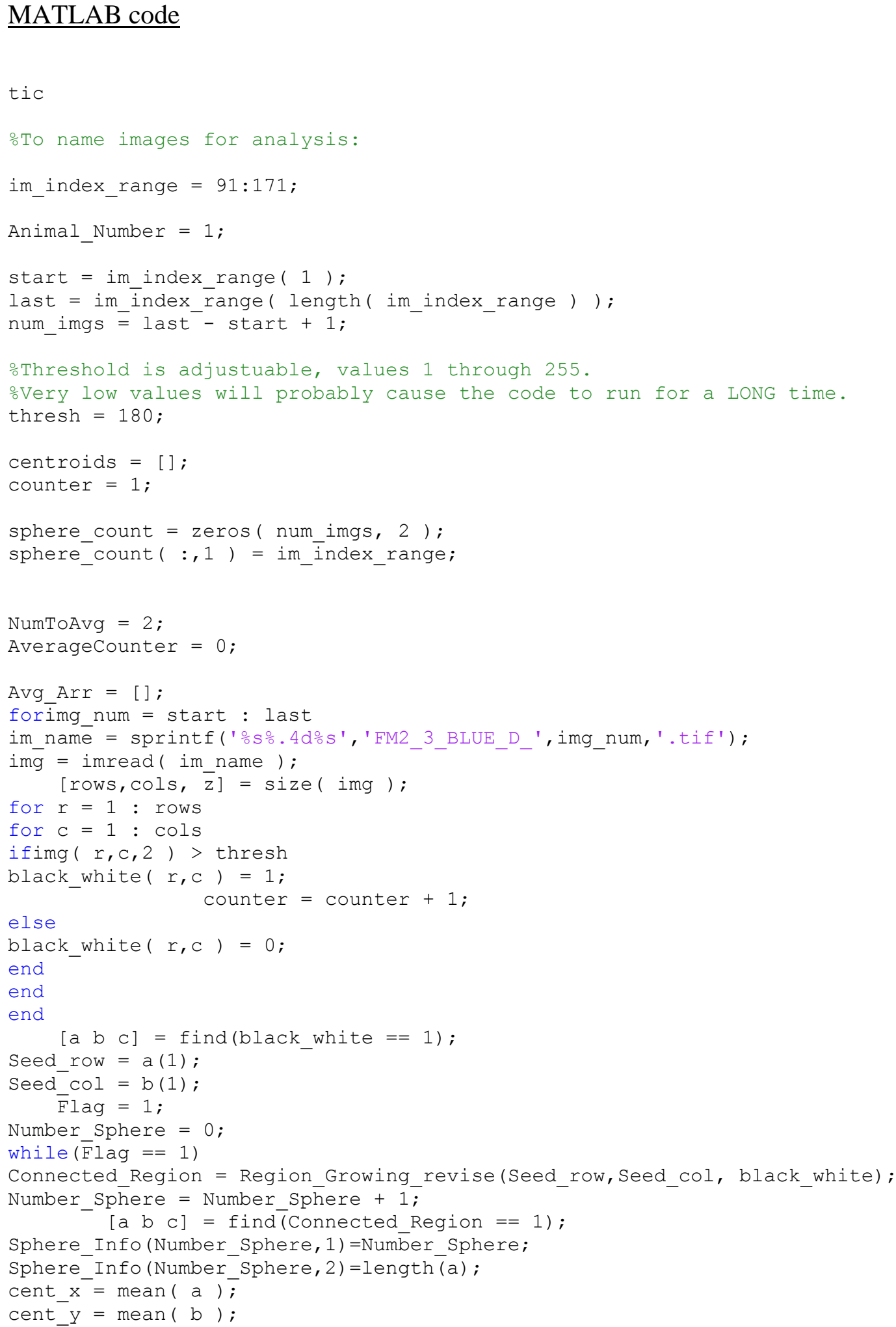

$\underline{\text { MATLAB code }}$

tic

oTo name images for analysis:

im_index_range $=91: 171$;

Animal_Number $=1$;

start $=$ im index range $(1)$;

last $=$ im_index_range ( length ( im_index_range ) );

num_imgs $=$ last - start +1 ;

oThreshold is adjustuable, values 1 through 255.

oVery low values will probably cause the code to run for a LONG time.

thresh $=180$

centroids $=[]$;

counter $=1$;

sphere count $=$ zeros ( num imgs, 2 );

sphere_count $(:, 1)=$ im_index_range;

NumToAvg $=2$;

AverageCounter $=0$;

$\operatorname{Avg} \operatorname{Arr}=[] ;$

forimg num = start : last

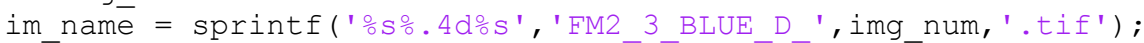

$i m \bar{g}=$ imread ( im_name) $;$

[rows, cols, z] = size( img );

for $r=1$ : rows

for $c=1$ : cols

ifimg $(r, c, 2)>$ thresh

black white $(r, c)=1$;

counter $=$ counter $+1 ;$

else

black_white $(r, c)=0$;

end

end

end

$\left[\begin{array}{lll}\mathrm{a} & \mathrm{b} & \mathrm{c}\end{array}\right]=$ find (black white $\left.==1\right)$;

Seed row $=a(1)$;

Seed $\operatorname{col}=\mathrm{b}(1)$

$\overline{\mathrm{F}}$ lag $=1$

Number Sphere $=0$;

while (Flag $==1)$

Connected Region = Region_Growing revise (Seed_row, Seed_col, black_white);

Number_Sphere $=$ Number_Sphere +1 ;

$\left[\begin{array}{lll}\mathrm{a} & \mathrm{b} & \mathrm{c}\end{array}\right]=\mathrm{find}$ (Connected Region $\left.==1\right)$;

Sphere_Info (Number_Sphere, 1 ) =Number_Sphere

Sphere_Info (Number_Sphere, 2) =length (a) ;

cent $\mathrm{x}=\operatorname{mean}(\mathrm{a})$;

cent ${ }^{-} \mathrm{y}=\operatorname{mean}(\mathrm{b})$; 


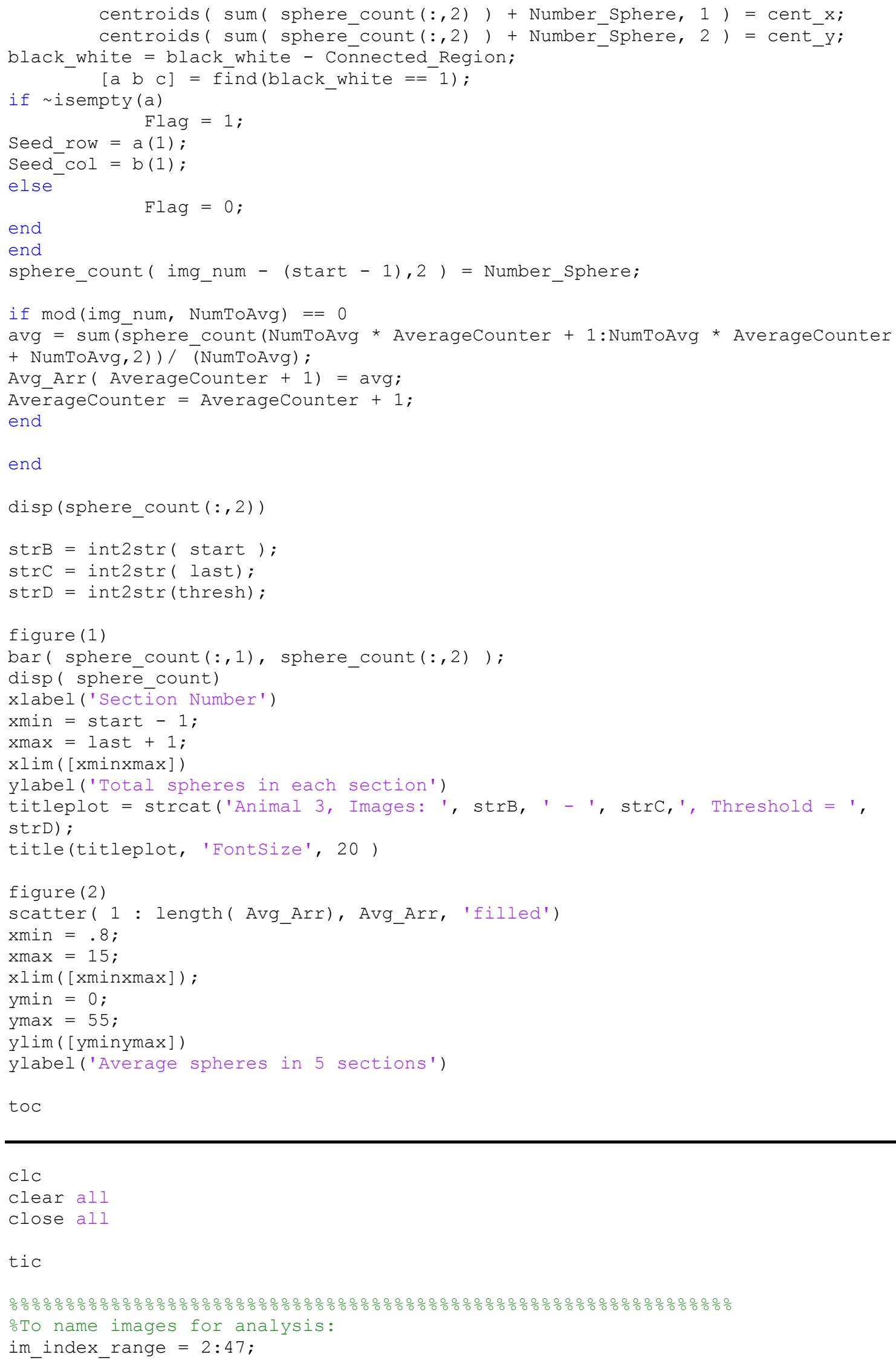




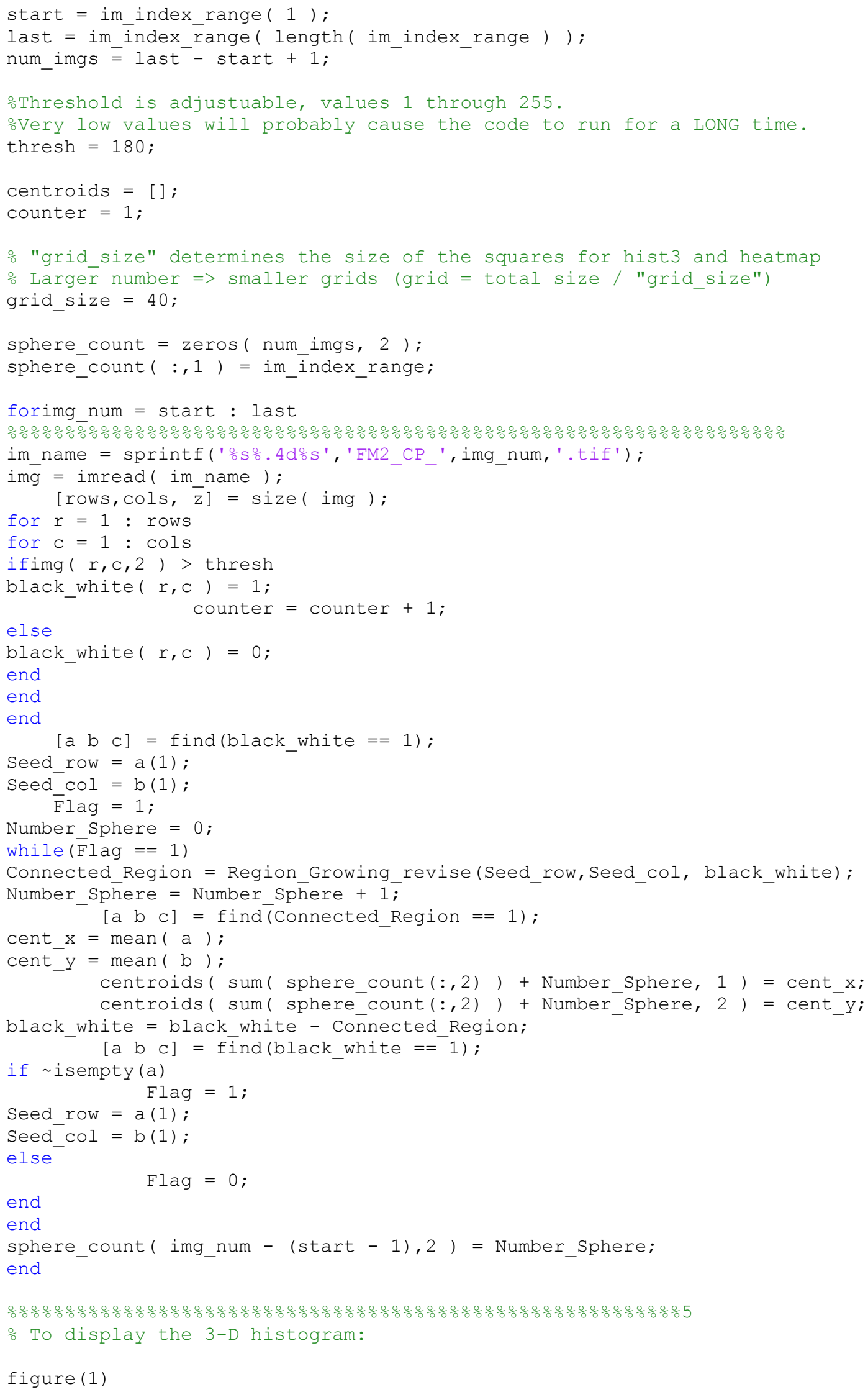


zlabel('Number of spheres per grid square')

xlabel('(coordinate 0,0 corresponds to upper left of image)')

set(gca, 'XTickLabel', \{' '\}) 응 To remove number labels on X, keep grid

lines, keep axis label

set (gca, 'YTickLabel', \{' '\}) 응 To remove number labels on Y, keep grid

lines, keep axis label

title('Display of sphere distribution in multiple tissue sections')

hold on

hist3( centroids, 'edges', \{0:rows/grid_size:rows; 0:cols/grid_size:cols \});

$\mathrm{n}=$ hist3( centroids, 'edges', \{0:rows/grid_size:rows; 0:cols/grid_size:cols \}

);

$\mathrm{n} 1=\mathrm{n}^{\prime}$;

$\mathrm{n} 1(\operatorname{size}(\mathrm{n}, 1)+1, \operatorname{size}(\mathrm{n}, 2)+1)=0$

$\mathrm{xb}=\operatorname{linspace}(\min (\operatorname{centroids}(:, 1)), \max (\operatorname{centroids}(:, 1)), \operatorname{size}(\mathrm{n}, 1)+1)$;

$\mathrm{yb}=$ linspace $(\min (\operatorname{centroids}(:, 2))$, max (centroids $(:, 2))$, size $(\mathrm{n}, 1)+1)$;

$\mathrm{h}=\operatorname{pcolor}(\mathrm{xb}, \mathrm{yb}, \mathrm{n} 1)$;

o Sets where the colormap goes

\% "-max" puts it beneath the 3D plot, max \# of counts distance

set ( h, 'zdata', ones(size(n1)) * $-\max (\max (n)))$;

colormap (hot)

grid on

view (3)

hold off

strA $=$ int2str $($ start $) ;$

strB $=$ int2str ( last $)$;

strC $=$ int2str(grid size);

strD = int2str(thresh);

figure (2)

$\mathrm{h}=\operatorname{pcolor}(\mathrm{xb}, \mathrm{yb}, \mathrm{n} 1)$;

set (gca, 'XTickLabel', $\{$ ' ' $\}$ )

set (gca,' 'YTickLabel', \{' '\})

titleplot = strcat ('Animal 2, Images: ', strA, ' - ', strB, ', Grid of: .', strC,', Threshold = ', strD);

title(titleplot)

toc

functionConnected_Region = Region_Growing_revise(Seed_row,Seed_col,Y);

$\mathrm{Y}=\mathrm{Y} \cdot{ }^{*} 255$

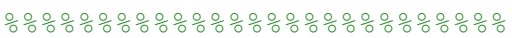

$[\mathrm{h} \mathrm{w}]=\operatorname{size}(\mathrm{Y})$;

Connected $\operatorname{Im}=\operatorname{zeros}(\mathrm{h}, \mathrm{w})$;

Index_Im $=\operatorname{zeros}(\mathrm{h}, \mathrm{w})$;

R_array (1) = Seed_row;

C-array $(1)$ = Seed col;

Iñdex Im (Seed row, Seed Col) = 1;

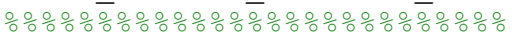

Visting Points $=0$;

counter $=1$;

while (counter =Visting Points)

Visting_Points $=\overline{\text { Visting_Points }}+1$;

[Visting Points counter];

$i=$ R_array (Visting_Points); 


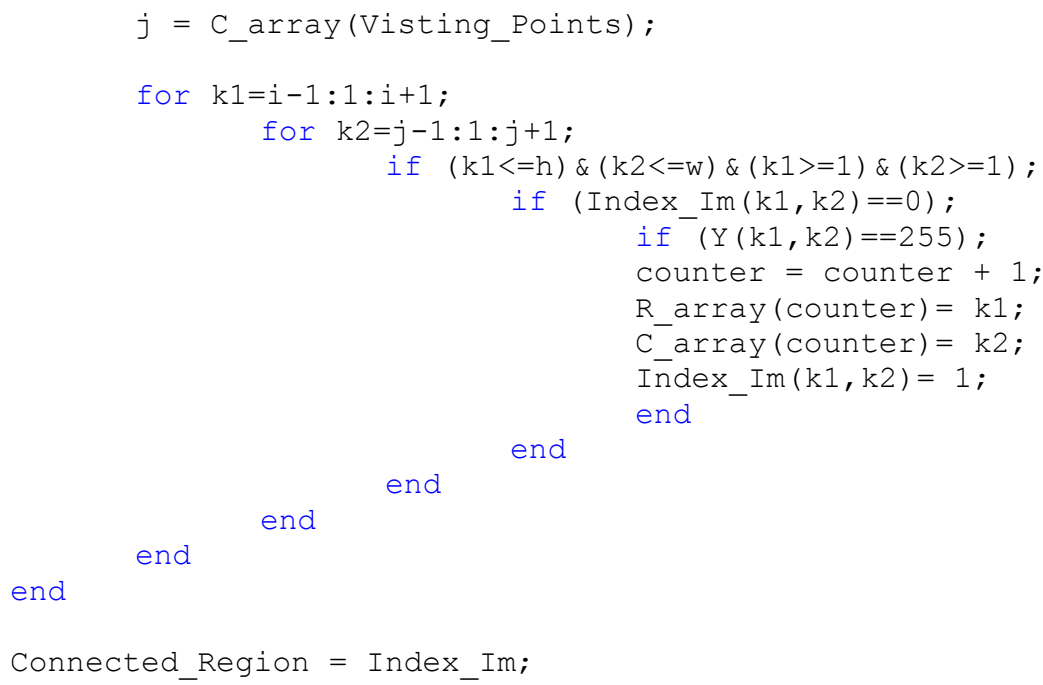

